\title{
N-Trifluoromethyl NHC Ligands Provide Selective Ruthenium Metathesis Catalysts
}

Pascal S. Engl, Alexey Fedorov, Christophe Copéret, Antonio Togni*

Swiss Federal Institute of Technology ETH Zürich, Department of Chemistry and Applied Biosciences, Vladimir Prelog Weg 2, CH-8093 Zürich, Switzerland. Tel: +41 44632 2236; E-mail: atogni@ethz.ch

\section{Supporting Information}




\section{Table of Contents}

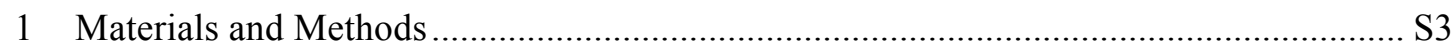

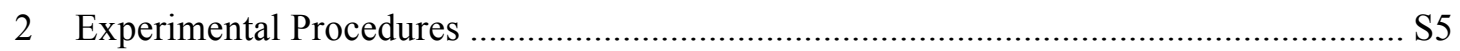

2.1 (3,5-BIS(TRIFLUOROMETHYL)PHENYL)(MESITYL)IODONIUM TETRAFLUOROBORATE (C) ......... S5

2.2 3-(3,5-BIS(TRIFLUOROMETHYL)PHENYL)-1-(TRIFLUOROMETHYL)-1 $H$-BENZO[D]IMIDAZOL-3IUM TETRAFLUOROBORATE $(5 \mathrm{C})$ S6

2.3 3-BUTYL-1-(TRIFLUOROMETHYL)-1 $H$-BENZO[D]IMIDAZOL-3-IUM

TRIFLUOROMETHANESULFONATE (5D)

2.4 1-ISOPROPYL-3-(4-(TRIFLUOROMETHYL)PHENYL)-1 $H$-BENZO[D]IMIDAZOL-3-IUM

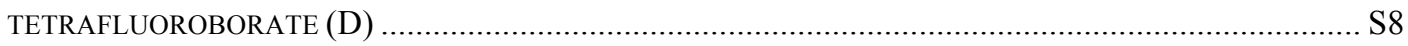

2.5 General Procedure A: Synthesis of Grubbs $1^{\text {st }}$ Generation Type CompleXes........... S9

2.5.1 Tricyclohexylphosphine[1-(trifluoromethyl)-3-(phenyl)--1H-benzo[d]imidazol-3ylidene] [benzylidene] ruthenium dichloride $(6 a)$.................................................................S9

2.5.2 Tricyclohexylphosphine[1-(trifluoromethyl)-3-(4-(trifluoromethyl)phe-nyl)-1H-

benzo[d] imidazol-3-ylidene] [benzylidene] ruthenium dichloride (6b) ...................................S10

2.5.3 Tricyclohexylphosphine [3-(3,5-bis(trifluoromethyl)phenyl)-1-(trifluoromethyl)-1H-

benzo[d]imidazol-3-ylidene] [benzylidene] ruthenium dichloride (6c)...................................S11

2.5.4 Tricyclohexylphosphine[3-butyl-1-(trifluoromethyl)-1H-benzo[d]imidazol-3-

ylidene] [benzylidene] ruthenium dichloride $(6 d)$............................................................... 11

2.5.5 Tricyclohexylphosphine[1-isopropyl-3-(4-(trifluoromethyl)phenyl)-1H-benzo[d]imidazol-

3-ylidene] [benzylidene] ruthenium dichloride (7) ...........................................................S12

2.6 General procedure B: Ring Closing Metathesis of Diethyl Diallylmalonate..... S13

2.7 General procedure C: Cross Metathesis of ALlyl BENZENE With CIS-1,4-DiacetoXy-2-

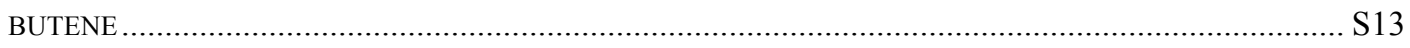

2.8 General procedure D: COPOLyMERIZATION OF Norbornene AND CIS-CyClOOCTENE. ${ }^{13} \mathrm{C}$

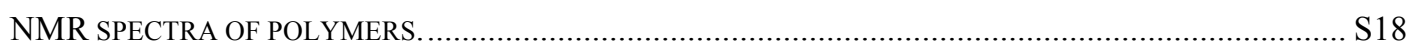

2.9 General PRocedure E: Ethenolysis of Ethyl Oleate................................................. S2

2.10 GENERAL PROCEDURE F: DETERMINATION OF ACTIVATION PARAMETERS ............................ S21

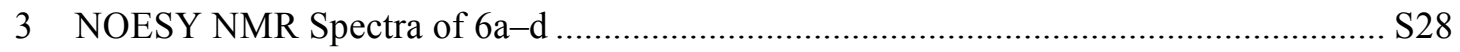

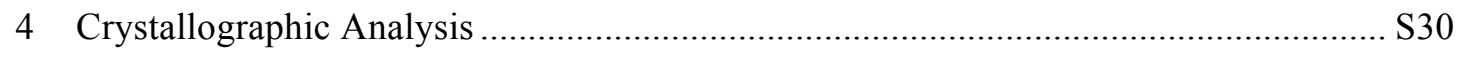

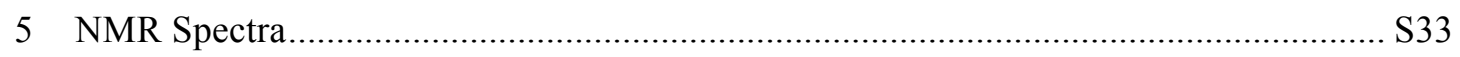

5.1 MESITYL(4-(TRIFLUOROMETHYL)PHENYL)IODONIUM TETRAFLUOROBORATE (C) ................. S33

5.2 3-(3,5-BIS(TRIFLUOROMETHYL)PHENYL)-1-(TRIFLUOROMETHYL)-1 $H$-BENZO[D]IMIDAZOL-3-

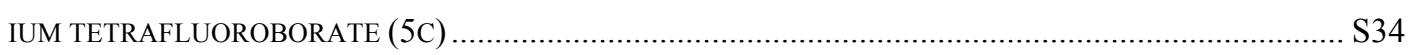

5.3 3-BUTYL-1-(TRIFLUOROMETHYL)-1 $H$-BENZO[D]IMIDAZOL-3-IUM

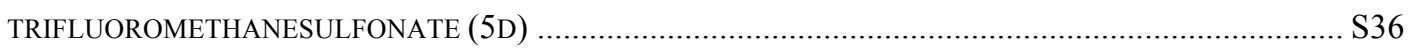

5.4 1-ISOPROPYL-3-(4-(TRIFLUOROMETHYL)PHENYL)-1 $H$-BENZO[ $D$ ]IMIDAZOL-3-IUM

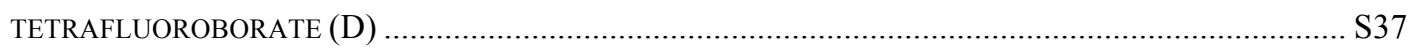

5.5 TRICYClOHEXYLPHOSPHINe[1-(TRIFLUOROMETHYL)-3-(PHENYL)- $1 H$-BENZO[D]IMIDAZOL-3-

YLIDENE][BENZYLIDENE]RUTHENIUM DICHLORIDE (6A) ......................................................... S39 
5.6 TRICYCLOHEXYLPHOSPHINE[1-(TRIFLUOROMETHYL)-3-(4-(TRIFLUOROMETHYL)PHENYL)-1 $H$ BENZO[D]IMIDAZOL-3-YLIDENE][BENZYLIDENE]RUTHENIUM DICHLORIDE (6B) .....

5.7 TRICYCLOHEXYLPHOSPHINE[3-(3,5-BIS(TRIFLUOROMETHYL)PHENYL)-1-(TRIFLUOROMETHYL)$1 H$-BENZO[D]IMIDAZOL-3-YLIDENE][BENZYLIDENE]RUTHENIUM DICHLORIDE (6C). S43

5.8 TRICYCLOHEXYLPHOSPHINE[3-BUTYL-1-(TRIFLUOROMETHYL)-1 $H$-BENZO[ $D$ ]IMIDAZOL-3YLIDENE][BENZYLIDENE]RUTHENIUM DICHLORIDE (6D) $\mathrm{S} 45$

5.9 TRICYCLOHEXYLPHOSPHINE[1-ISOPROPYL-3-(4-(TRIFLUOROMETHYL)PHENYL)- $1 \mathrm{H}$ BENZO $[D]$ IMIDAZOL-3-YLIDENE][BENZYLIDENE]RUTHENIUM DICHLORIDE (7). S47

6 References S49

\section{Materials and Methods}

All experiments were performed under an argon atmosphere using standard Schlenk techniques. All solvents were distilled and dried prior to utilization $\left(\mathrm{CH}_{2} \mathrm{Cl}_{2}\right.$ from $\mathrm{CaH}_{2}$; hexane and toluene from Na/benzophenone). Toluene (Sigma Aldrich, 99.9\%) for the catalytic cross metathesis and ethenolysis experiments was saturated with $\mathrm{N}_{2}$ and then purified by passage through double solvent purification alumina columns (MBraun) and stored over activated Selexsorb CD ${ }^{\circledR}$ (BASF). Unless otherwise stated all commercially available chemicals were used without further purification. Allylbenzene (Sigma Aldrich, 98\%), cis-1,4-diacetoxybutene (Alfa Aesar, 96\%), ntridecane (Sigma Aldrich, 99\%) and ethyl vinyl ether (Sigma Aldrich, 99\%) were degassed by 3 freeze-pump-thaw cycles. Cis-cyclooctene (Tokyo Chemical Industrie, 95\%) was distilled from $\mathrm{Na}$ prior to use. Ethyl oleate (Nu-Chek-prep, $>99 \%$ ) was degassed by three freeze-pump-thaw cycles and passed through a column with activated alumina under nitrogen atmosphere and stored over activated Selexsorb $C^{\circledR}$ (BASF) sorbent in a freezer. The following reagents were synthesized according to reported procedures: 1-(trifluoromethyl)-1H-benzo[ $[d]$ imidazole and benzimidazolium salts 5a-b. ${ }^{1}$ Silica gel 60 (230-400 mesh) was purchased from Fluka. TLC plates were obtained from Merck (silica gel 60 F254). NMR spectra were recorded on a Bruker DPX-200, DPX-300, DPX-400 and DPX-500 instruments operating at the denoted spectrometer frequency given in $\mathrm{MHz}$ for the specified nucleus. The ${ }^{1} \mathrm{H}$ and ${ }^{13} \mathrm{C}$ chemical shifts are referred to TMS and calibrated with the residual solvent peak. For ${ }^{19} \mathrm{~F}$ NMR, $\mathrm{CFCl}_{3}$ was used as an external standard. The chemical shifts are reported in 
parts per million (ppm) and coupling constants $(J)$ are given in Hertz $(\mathrm{Hz})$. High resolution mass spectra were measured by the MS-service of the "Laboratorium für Organische Chemie der ETH Zürich”. Values are given as $\mathrm{m} / \mathrm{z}$ and the intensity $\mathrm{I} \%$ of the base peak. Elemental analysis was performed by the microelemental analysis service of the "Laboratorium für Organische Chemie der ETH Zürich the Mikroelementalanalytisches Laboratorium der ETH Zürich”. Melting points were determined on a Büchi Melting Point B-540 apparatus in open capillaries and are uncorrected. IR spectra were recorded on a Thermo Fischer Scientific Nicolet 6700 FTIR equipped with a PIKE technologies GladiATR ${ }^{\mathrm{TM}}$ or on a Perkin-Elmer BX II using ATR FT-IR technology and are reported as absorption maxima in $\mathrm{cm}^{-1}$. X-ray diffraction structural analysis was carried out on a Siemens CCD diffractometer (Siemens SMART PLATFORM, with CCD detector, graphite monochromator, Mo-K ${ }_{\alpha^{-}}$ radiation).

The catalytic cross metathesis experiments were performed at the High Throughput Experimentation facility of the ETH Zürich (HTE@ETH) on a custom-made robotic platform operated inside a nitrogen-filled glove box with $\mathrm{O}_{2}$ and $\mathrm{H}_{2} \mathrm{O}$ levels $<0.1 \mathrm{ppm}$. Ethenolysis reactions were carried out on an Endeavour autoclave system (Biotage) operated inside a glove box. The ethylene feed was passed through activated $\mathrm{Cu} / \mathrm{Al}_{2} \mathrm{O}_{3}$ sorbent (BASF) and activated $4 \AA$ molecular sieves before entering the autoclave system. Gas chromatography was performed on a Thermo Fischer Trace GC 2000 equipped with a flame ionization detector, using a ZB-5 column with guardian (L: 30 m, i.d.: $0.25 \mathrm{~mm}, \mathrm{DF}=0.25 \mu \mathrm{m}$ ) and helium as the carrier gas with a constant flow of $1.1 \mathrm{~mL} \mathrm{~min}^{-1}$. 


\section{Experimental Procedures}

\section{1 (3,5-bis(trifluoromethyl)phenyl)(mesityl)iodonium tetrafluoroborate (C)}

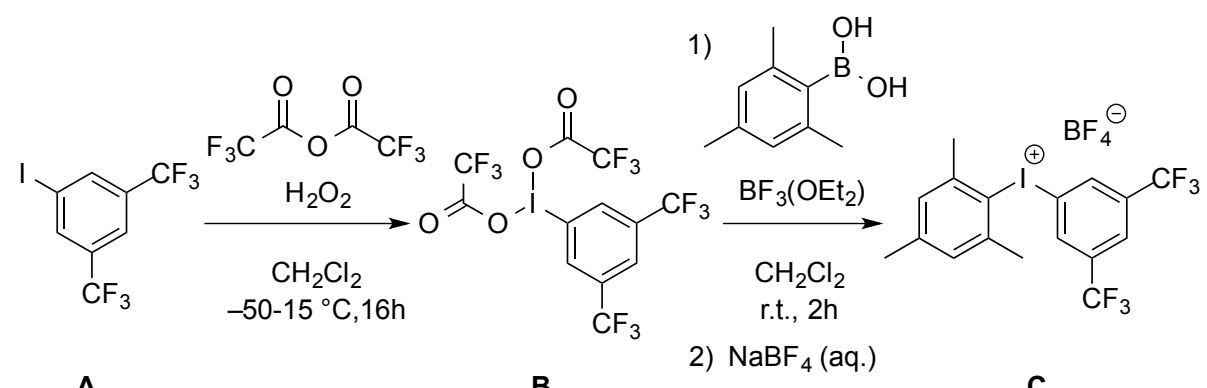

A $250 \mathrm{~mL}$ round bottom flask was charged with $\mathrm{CH}_{2} \mathrm{Cl}_{2}(100 \mathrm{~mL})$ and trifluoroacetic anhydride $(80 \mathrm{~mL})$. The solution was then cooled to $-50{ }^{\circ} \mathrm{C}$ and $\mathrm{H}_{2} \mathrm{O}_{2}(30 \mathrm{wt} \%, 16 \mathrm{~mL})$ was added drop wise. Subsequently, 1-iodo-3,5-bis(trifluoromethyl)benzene A (4.08 g, $12 \mathrm{mmol}, 1$ equiv) was added via spatula and the reaction mixture was allowed to warm up to room temperature over a period of 16 hours. The reaction mixture was then concentrated to dryness in vacuo affording (3,5-bis(trifluoromethyl)phenyl)- $\lambda^{3}$ iodanediyl bis(2,2,2-trifluoroacetate) $\mathbf{B}$ as white solid, which was used without further purification. A $500 \mathrm{~mL}$ round bottom flask was charged with solution of mesitylboronic acid (1.865 g, $13.2 \mathrm{mmol}, 1.1$ equiv) in $\mathrm{CH}_{2} \mathrm{Cl}_{2}(170 \mathrm{~mL})$ and cooled to $0{ }^{\circ} \mathrm{C}$. Subsequently, $\mathrm{BF}_{3} \cdot \mathrm{OEt}_{2}(1.48 \mathrm{~mL}, 13.2 \mathrm{mmol}, 1.1$ equiv $)$ was added and the reaction mixture was stirred for 2 hours at room temperature. The reaction was quenched by slow addition of a saturated aqueous solution of $\mathrm{NaBF}_{4}(250 \mathrm{~mL})$ and stirred for an additional $45 \mathrm{~min}$. The aqueous phase was then extracted with $\mathrm{CH}_{2} \mathrm{Cl}_{2}(3 \mathrm{x}$ $150 \mathrm{~mL}$ ). The combined organic phases were dried over $\mathrm{MgSO}_{4}$ and concentrated to dryness. Recrystallization of the residue from $\mathrm{CH}_{2} \mathrm{Cl}_{2} / \mathrm{Et}_{2} \mathrm{O}$ afforded mesityl(4(trifluoromethyl)phenyl)iodonium tetrafluoroborate $\mathbf{C}(2.463 \mathrm{~g}, 4.51 \mathrm{mmol}, 38 \%)$ as a white solid. ${ }^{1} \mathrm{H}$ NMR (400.20 MHz, DMSO-d $\left.\mathrm{d}_{6}\right): \delta=8.61(\mathrm{~s}, 2 \mathrm{H}, \mathrm{ArH}), 8.43(\mathrm{~s}, 1 \mathrm{H}$, ArH), 7.26 (s, 2H, ArH), $2.62\left(\mathrm{~s}, 6 \mathrm{H}, 2 \mathrm{x} \mathrm{CH}_{3}\right), 2.31\left(\mathrm{~s}, 3 \mathrm{H}, \mathrm{CH}_{3}\right) .{ }^{19} \mathrm{~F}\left\{{ }^{1} \mathrm{H}\right\} \mathrm{NMR}$ $\left(376.56 \mathrm{MHz}, \mathrm{DMSO}-\mathrm{d}_{6}\right): \delta=-61.36\left(\mathrm{~s}, \mathrm{ArCF}_{3}\right),-148.27\left(\mathrm{~s}, \mathrm{BF}_{4}\right) .{ }^{13} \mathrm{C}\left\{{ }^{1} \mathrm{H}\right\} \mathrm{NMR}$ $\left(100.63 \mathrm{MHz}, \mathrm{DMSO}-\mathrm{d}_{6}\right): \delta=143.73\left(\mathrm{C}_{\mathrm{Ar}}\right), 142.03\left(\mathrm{C}_{\mathrm{Ar}}\right), 134.98$ (apt d, $J=3.8 \mathrm{~Hz}, 2 \mathrm{x}$ $\left.\mathrm{CH}_{\mathrm{Ar}}\right), 132.27$ (q, $J=34.0 \mathrm{~Hz}, 2 \mathrm{x} \mathrm{C}$ Ar), $129.99\left(2 \mathrm{x} \mathrm{CH}_{\mathrm{Ar}}\right), 125.84$ (apt p, $J=4.0 \mathrm{~Hz}$, $\left.\mathrm{CH}_{\mathrm{Ar}}\right), 122.73\left(\mathrm{C}_{\mathrm{Ar}}\right), 122.15\left(\mathrm{q},{ }^{1} J_{\mathrm{CF}}=273.7 \mathrm{~Hz}, \mathrm{CF}_{3}\right), 115.56\left(\mathrm{C}_{\mathrm{Ar}}\right), 26.31\left(2 \mathrm{x} \mathrm{CH}_{3}\right)$, $20.53\left(\mathrm{CH}_{3}\right)$. IR (neat): 1662.3, 1452.4, 1345.9, 1277.8, 1134.1, 1036.7 (br), 887.2, 
$690.6 \mathrm{~cm}^{-1}$. HRMS (ESI): $\left[\mathrm{M}^{+}-\mathrm{BF}_{4}^{-}\right]$calcd for $\mathrm{C}_{17} \mathrm{H}_{14} \mathrm{~F}_{6} \mathrm{I}$ 459.00; found 459.0038. Anal. Calcd (\%) for $\mathrm{C}_{17} \mathrm{H}_{14} \mathrm{BF}_{10} \mathrm{I}$ : C, 37.40; H, 2.58. Found: C, 37.56; H, 2.66. Mp: $197.0-198.2^{\circ} \mathrm{C}$ with decomposition.

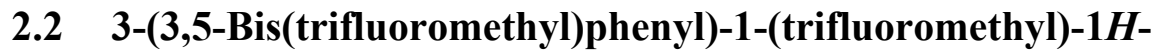 benzo[d] imidazol-3-ium tetrafluoroborate $(5 \mathrm{c})$}

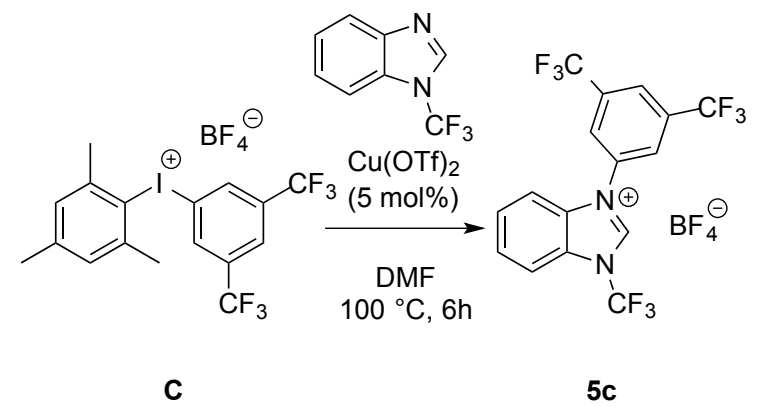

A $100 \mathrm{~mL}$ Schlenk flask equipped with a Teflon coated magnetic stirrer was charged with C (2.463 g, $4.51 \mathrm{mmol}, 1.5$ equiv) and DMF (10 mL). Subsequently, a solution of 1-(trifluoromethyl)-1H-benzo[ $[d$ imidazole $(560 \mathrm{mg}, 3.01 \mathrm{mmol}, 1$ equiv) in DMF (4 $\mathrm{mL}$ ) was added, followed by $\mathrm{Cu}(\mathrm{OTf})_{2}(39.4 \mathrm{mg}, 0.15 \mathrm{mmol}, 0.05$ equiv). The resulting reaction mixture was stirred at $100{ }^{\circ} \mathrm{C}$ for 6 hours. All volatiles were then removed under reduced pressure $\left(5 \cdot 10^{-3}\right.$ mbar $)$. Recrystallization of the residue from acetone $/ \mathrm{Et}_{2} \mathrm{O}$ afforded $\mathbf{5 c}(571 \mathrm{mg}, 1.17 \mathrm{mmol}, 39 \%)$ as a white solid. ${ }^{1} \mathrm{H}$ NMR (500.26 $\mathrm{MHz}$, acetone- $\left.\mathrm{d}_{6}\right): \delta=10.79(\mathrm{~s}, 1 \mathrm{H}, \mathrm{NCHN}), 8.73(\mathrm{~s}, 2 \mathrm{H}, \mathrm{ArH}), 8.56(\mathrm{~s}, 1 \mathrm{H}, \mathrm{ArH}), 8.28$ $\left(\mathrm{d},{ }^{3} J_{\mathrm{HH}}=8.5 \mathrm{~Hz}, 1 \mathrm{H}, \mathrm{ArH}\right), 8.12\left(\mathrm{~d},{ }^{3} J_{\mathrm{HH}}=8.4 \mathrm{~Hz}, 1 \mathrm{H}, \mathrm{ArH}\right), 8.04\left(\mathrm{t},{ }^{3} J_{\mathrm{HH}}=7.9 \mathrm{~Hz}\right.$, $1 \mathrm{H}, \mathrm{ArH}), 7.97\left(\mathrm{t},{ }^{3} J_{\mathrm{HH}}=7.9 \mathrm{~Hz}, 1 \mathrm{H}, \mathrm{ArH}\right) .{ }^{19} \mathrm{~F}\left\{{ }^{1} \mathrm{H}\right\}$ NMR (470.67 MHz, acetone- $\left.\mathrm{d}_{6}\right)$ : $\delta=-59.05\left(\mathrm{~s}, \mathrm{NCF}_{3}\right),-63.37\left(\mathrm{~s}, 2 \mathrm{x} \mathrm{ArCF}_{3}\right),-151.88\left(\right.$ br s, $\left.\mathrm{BF}_{4}\right) \cdot{ }^{13} \mathrm{C}\left\{{ }^{1} \mathrm{H}\right\} \mathrm{NMR}(75.48$ $\left.\mathrm{MHz}, \mathrm{MeOD}-\mathrm{d}_{4}\right): \delta=144.02(\mathrm{q}, J=3.3 \mathrm{~Hz}, \mathrm{NCHN}), 134.83\left(\mathrm{C}_{\mathrm{Ar}}\right), 134.19$ (q, $J=34.6$ $\left.\mathrm{Hz}, 2 \mathrm{x} \mathrm{C}_{\mathrm{Ar}}\right), 133.41\left(\mathrm{C}_{\mathrm{Ar}}\right), 130.88\left(\mathrm{CH}_{\mathrm{Ar}}\right), 130.40\left(\mathrm{CH}_{\mathrm{Ar}}\right), 128.85$ (q, $J=3.9 \mathrm{~Hz}, 2 \mathrm{x}$ $\left.\mathrm{CH}_{\mathrm{Ar}}\right), 128.25\left(\mathrm{C}_{\mathrm{Ar}}\right), 126.71\left(\mathrm{q}, J=3.7 \mathrm{~Hz}, \mathrm{CH}_{\mathrm{Ar}}\right), 123.58\left(\mathrm{q},{ }^{1} J_{\mathrm{CF}}=272.5 \mathrm{~Hz}, \mathrm{ArCF}_{3}\right)$, $118.41\left(\mathrm{q},{ }^{1} J_{\mathrm{CF}}=269.7 \mathrm{~Hz}, \mathrm{NCF}_{3}\right), 115.76\left(\mathrm{CH}_{\mathrm{Ar}}\right), 114.58\left(\mathrm{q}, J=1.6 \mathrm{~Hz}, \mathrm{CH}_{\mathrm{Ar}}\right)$. IR (neat): 3142.3, 3076.5, 3044.2, 1565.9, 1383.9, 1281.2, 1140.3, 1062.8, 1038.5, 1008.7, 906.8, 746.2 $\mathrm{cm}^{-1}$. HRMS (ESI): $\left[\mathrm{M}^{+}-\mathrm{BF}_{4}\right]$ calcd for $\mathrm{C}_{16} \mathrm{H}_{8} \mathrm{~F}_{9} \mathrm{~N}_{2}$ 399.05; found 399.0538. Mp: $186.8-188.1^{\circ} \mathrm{C}$. 


\subsection{3-Butyl-1-(trifluoromethyl)-1H-benzo $[d]$ imidazol-3-ium trifluoromethanesulfonate $(5 \mathrm{~d})$}

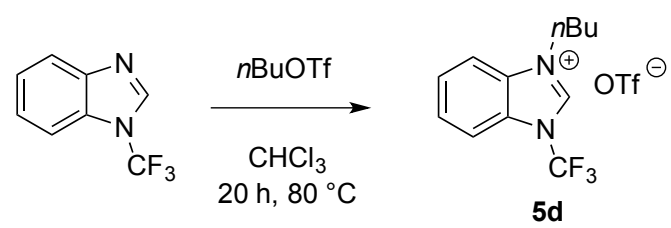

A $50 \mathrm{ml}$ Young Schlenk flask equipped with a Teflon coated magnetic stirrer was charged with 1-(trifluoromethyl)-1H-benzo[d]imidazole ( $1 \mathrm{~g}, 5.37 \mathrm{mmol}, 1$ equiv) and $\mathrm{CH}_{3} \mathrm{Cl}(6 \mathrm{~mL})$. Then, a solution of $n$-butyl trifluoromethanesulfonate $(0.95 \mathrm{ml}, 8.06$ mmol, 1.5 equiv) in $\mathrm{CH}_{3} \mathrm{Cl}(4 \mathrm{ml})$ was added and the reaction mixture was stirred at 80 ${ }^{\circ} \mathrm{C}$ for $20 \mathrm{~h}$. All volatiles were then removed under reduced pressure $\left(9 \cdot 10^{-3} \mathrm{mbar}\right)$. Recrystallization of the oily residue from acetone/ $\mathrm{Et}_{2} \mathrm{O}$ afforded $\mathbf{5 d}(950.6 \mathrm{mg}, 2.42$ mmol, $45 \%)$ as a white solid. ${ }^{1} \mathrm{H}$ NMR (300.13 MHz, DMSO-d 6 ): $\delta=10.65(\mathrm{~s}, 1 \mathrm{H}$, NCHN), 8.29 (dd, $J=6.4,2.9 \mathrm{~Hz}, 1 \mathrm{H}, \mathrm{ArH}), 8.13-8.09$ (m, 1H, ArH), $7.90-7.84$ (m, $2 \mathrm{H}, \mathrm{ArH}), 4.57\left(\mathrm{t},{ }^{3} J_{\mathrm{HH}}=7.4 \mathrm{~Hz}, 2 \mathrm{H}, \mathrm{NCH}_{2}\left(\mathrm{CH}_{2}\right)_{2} \mathrm{CH}_{3}\right), 1.97\left(\mathrm{p},{ }^{3} J_{\mathrm{HH}}=7.6 \mathrm{~Hz}, 2 \mathrm{H}\right.$, $\left.\mathrm{CH}_{2} \mathrm{CH}_{2} \mathrm{CH}_{3}\right), 1.44\left(\mathrm{dq},{ }^{3} J_{\mathrm{HH}}=14.7,{ }^{3} J_{\mathrm{HH}}=7.3 \mathrm{~Hz}, 2 \mathrm{H}, \mathrm{CH}_{2} \mathrm{CH}_{2} \mathrm{CH}_{3}\right), 0.96\left(\mathrm{t},{ }^{3} J_{\mathrm{HH}}=\right.$ $\left.7.3 \mathrm{~Hz}, 3 \mathrm{H}, \mathrm{CH}_{3}\right) .{ }^{19} \mathrm{~F}\left\{{ }^{1} \mathrm{H}\right\} \mathrm{NMR}\left(282.38 \mathrm{MHz}, \mathrm{DMSO}-\mathrm{d}_{6}\right): \delta=-57.43\left(\mathrm{~s}, \mathrm{NCF}_{3}\right),-$ 77.79 (s, OTf). ${ }^{13} \mathrm{C}\left\{{ }^{1} \mathrm{H}\right\}$ NMR (75.48 MHz, DMSO-d 6$): \delta=143.01(\mathrm{~d}, J=3.1 \mathrm{~Hz}$, $\mathrm{NCHN}), 131.28\left(\mathrm{C}_{\mathrm{Ar}}\right), 128.98\left(\mathrm{CH}_{\mathrm{Ar}}\right), 128.10\left(\mathrm{CH}_{\mathrm{Ar}}\right), 126.96\left(\mathrm{C}_{\mathrm{Ar}}\right), 120.66\left(\mathrm{q},{ }^{1} J_{\mathrm{CF}}=\right.$ $322.4 \mathrm{~Hz}, \mathrm{OTf}), 117.01$ (q, $\left.{ }^{1} J_{\mathrm{CF}}=268.1 \mathrm{~Hz}, \mathrm{NCF}_{3}\right), 114.96\left(\mathrm{CH}_{\mathrm{Ar}}\right), 113.34\left(\mathrm{CH}_{\mathrm{Ar}}\right)$, $47.73\left(\mathrm{NCH}_{2}\right), 30.16\left(\mathrm{CH}_{2}\right), 19.09\left(\mathrm{CH}_{2}\right), 13.38\left(\mathrm{CH}_{3}\right) . \mathrm{IR}$ (neat): 3149.9, 3083.8, 2973.4, 2880.4, 1568.4, 1352.5, 1259.7, 1202.8, 1163.4, 1081.6, 1025.9, 759.8, 632.8 $\mathrm{cm}^{-1}$. HRMS (ESI): [ $\mathrm{M}^{+}-\mathrm{CF}_{3} \mathrm{SO}_{3}$ ] calcd for $\mathrm{C}_{12} \mathrm{H}_{14} \mathrm{~F}_{3} \mathrm{~N}_{2}$ 243.11; found 243.1105. Anal. Calcd (\%) for $\mathrm{C}_{13} \mathrm{H}_{14} \mathrm{~N}_{2} \mathrm{O}_{3} \mathrm{~F}_{6} \mathrm{~S}: \mathrm{C}, 39.80 ; \mathrm{H}, 3.60 ; \mathrm{N}, 7.14$. Found: C, 40.77; H, 3.54; N, 7.04 (probably, solvent impurities accounted for the deviation in carbon content). Mp: $169.1-170.1^{\circ} \mathrm{C}$. 


\subsection{1-Isopropyl-3-(4-(trifluoromethyl)phenyl)-1H-benzo[d]imidazol-3-ium tetrafluoroborate (D)}
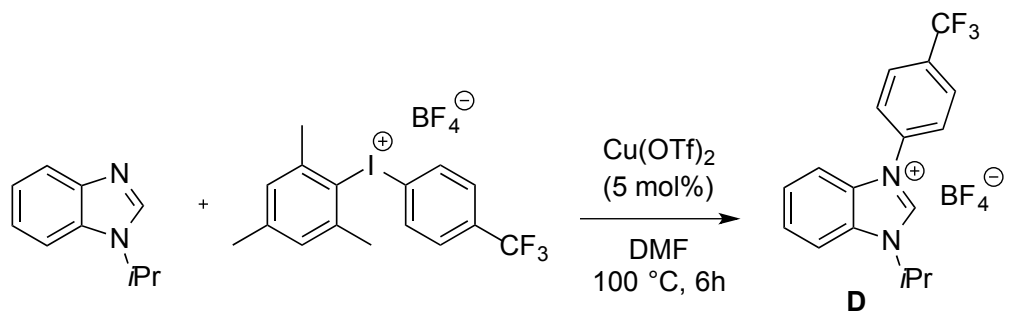

A $100 \mathrm{~mL}$ Schlenk flask equipped with a Teflon coated magnetic stirrer was charged with mesityl(4-(trifluoromethyl)phenyl)iodonium tetrafluoroborate $(2.041 \mathrm{~g}, 4.68$ mmol, 1.5 equiv) and DMF (10 mL). Subsequently, a solution of 1 -isopropyl- $1 H$ benzo[d]imidazole (500 mg, $3.12 \mathrm{mmol}, 1$ equiv) in DMF (4 mL) was added, followed by $\mathrm{Cu}(\mathrm{OTf})_{2}(56.4 \mathrm{mg}, 0.16 \mathrm{mmol}, 0.05$ equiv). The resulting reaction mixture was stirred at $100{ }^{\circ} \mathrm{C}$ for 6 hours. All volatiles were then removed under reduced pressure $\left(5 \cdot 10^{-3}\right.$ mbar $)$. The residue was purified by flash column chromatography (DCM/acetone 5:1 - 2:1 v/v), followed by subsequent recrystallization from acetone/ $\mathrm{Et}_{2} \mathrm{O}$ to afford $\mathbf{D}(1.089 \mathrm{~g}, 2.78 \mathrm{mmol}, 89 \%)$ as a white solid. ${ }^{1} \mathrm{H}$ NMR (500.26 MHz, DMSO-d $\left.{ }_{6}\right): \delta=10.24(\mathrm{~s}, 1 \mathrm{H}, \mathrm{NCHN}), 8.28\left(\mathrm{~d},{ }^{3} J_{\mathrm{HH}}=8.0 \mathrm{~Hz}, 1 \mathrm{H}, \mathrm{ArH}\right), 8.19-$ $8.12(\mathrm{~m}, 4 \mathrm{H}, \mathrm{ArH}), 7.94\left(\mathrm{~d},{ }^{3} \mathrm{~J}_{\mathrm{HH}}=7.9 \mathrm{~Hz}, 1 \mathrm{H}, \mathrm{ArH}\right), 7.78$ (apt dt, $J=23.5,7.0 \mathrm{~Hz}, 2 \mathrm{H}$, $\mathrm{ArH}), 5.19\left(\mathrm{apt} \mathrm{dt},{ }^{3} J_{\mathrm{HH}}=13.4,{ }^{3} J_{\mathrm{HH}}=6.9 \mathrm{~Hz}, 1 \mathrm{H}, \mathrm{NCH}\left(\mathrm{CH}_{3}\right)_{2}\right), 1.72\left(\mathrm{~d},{ }^{3} J_{\mathrm{HH}}=6.7 \mathrm{~Hz}\right.$ $\left.\mathrm{NCH}\left(\mathrm{CH}_{3}\right)_{2}\right) .{ }^{19} \mathrm{~F}\left\{{ }^{1} \mathrm{H}\right\} \mathrm{NMR}\left(282.38 \mathrm{MHz}, \mathrm{DMSO}-\mathrm{d}_{6}\right): \delta=-61.16\left(\mathrm{~s}, \mathrm{ArCF}_{3}\right),-148.34$ $\left(\mathrm{s}, \mathrm{BF}_{4}\right) .{ }^{13} \mathrm{C}\left\{{ }^{1} \mathrm{H}\right\}$ NMR $\left(125.80 \mathrm{MHz}, \mathrm{DMSO}-\mathrm{d}_{6}\right): \delta=141.45(\mathrm{NCHN}), 136.66\left(\mathrm{C}_{\mathrm{Ar}}\right)$, $131.06\left(\mathrm{C}_{\mathrm{Ar}}\right), 130.65\left(\mathrm{C}_{\mathrm{Ar}}\right), 130.45\left(\mathrm{q}, J=32.5 \mathrm{~Hz}, \mathrm{C}_{\mathrm{Ar}}\right), 127.60\left(\mathrm{CH}_{\mathrm{Ar}}\right), 127.45(\mathrm{q}, J=$ $\left.3.5 \mathrm{~Hz}, 2 \mathrm{x} \mathrm{CH} \mathrm{Cr}_{\mathrm{Ar}}\right), 127.03\left(\mathrm{CH}_{\mathrm{Ar}}\right), 126.52\left(2 \mathrm{x} \mathrm{CH}_{\mathrm{Ar}}\right), 123.69\left(\mathrm{q},{ }^{1} J_{\mathrm{CF}}=272.6 \mathrm{~Hz}, \mathrm{CF}_{3}\right)$, $114.37\left(\mathrm{CH}_{\mathrm{Ar}}\right), 113.55\left(\mathrm{CH}_{\mathrm{Ar}}\right), 51.17(\mathrm{~N}-\mathrm{CH}), 21.49\left(2 \mathrm{x} \mathrm{CH}_{3}\right)$. IR (neat): 3153.9, 3098.1, 2982.1, 2943.6, 1566.1, 1318.4, 1247.9, 1114.3, 1059.4, 1012.8, 844.6, 746.7 $\mathrm{cm}^{-1}$. HRMS (ESI): $\left[\mathrm{M}^{+}-\mathrm{BF}_{4}\right]$ calcd for $\mathrm{C}_{17} \mathrm{H}_{16} \mathrm{~F}_{3} \mathrm{~N}_{2}$ 305.13; found 305.1262. Anal. Calcd (\%) for $\mathrm{C}_{13} \mathrm{H}_{14} \mathrm{~N}_{2} \mathrm{O}_{3} \mathrm{~F}_{6} \mathrm{~S}: \mathrm{C}, 52.07 ; \mathrm{H}, 4.11 ; \mathrm{N}, 7.14$. Found: C, 51.60; H, 3.95; N, 6.99. Mp: $144.9-145.9^{\circ} \mathrm{C}$. 


\subsection{General Procedure A: Synthesis of Grubbs $1^{\text {st }}$ Generation Type Complexes}

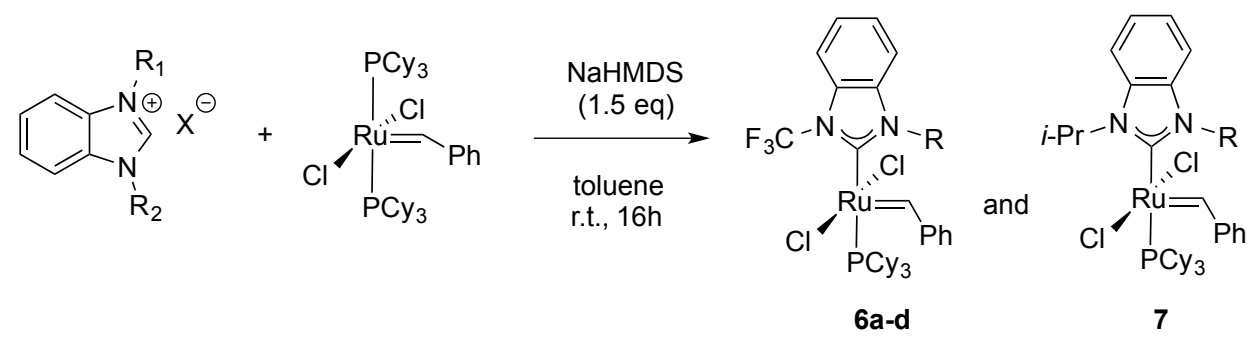

In a glove box, the corresponding benzimidazolium salt ( 1 equiv), Grubbs $1^{\text {st }}$ generation ( 1 equiv) and toluene $(0.1 \mathrm{M})$ were added to a Schlenk flask equipped with a Teflon coated magnetic stirrer. Subsequently, NaHMDS (1.5 equiv) was added and the resulting reaction mixture was stirred at room temperature for 16 hours. The reaction mixture was then filtered via cannula and concentrated to dryness under reduced pressure. The remaining residue was washed with hexane and then recrystallized from DCM/hexane to afford the desired ruthenium complex.

\subsubsection{Tricyclohexylphosphine[1-(trifluoromethyl)-3-(phenyl)--1H-} benzo[d]imidazol-3-ylidene][benzylidene]ruthenium dichloride (6a)

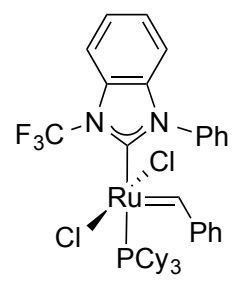

$6 a$

Synthesized according to general procedure A from 5a $(250 \mathrm{mg}, 0.61$ mmol), Grubbs $1^{\text {st }}$ generation catalyst $(500 \mathrm{mg}, 0.61 \mathrm{mmol})$. The product was obtained as a pale green solid $(225.7 \mathrm{mg}, 0.28 \mathrm{mmol}$, $46 \%$ ). ${ }^{1} \mathrm{H} \mathrm{NMR}\left(500.27 \mathrm{MHz}, \mathrm{CD}_{2} \mathrm{Cl}_{2}\right): \delta=19.24$ (s, 1H, RuCHPh), 8.45-6.26 (br s, 4H, ArH), 7.69 (apt d, $\left.{ }^{3} J_{\mathrm{HH}}=8.3 \mathrm{~Hz}, 1 \mathrm{H}, \mathrm{ArH}\right), 7.40$ (apt d, $\left.{ }^{3} J_{\mathrm{HH}}=7.5 \mathrm{~Hz}, 2 \mathrm{H}, \mathrm{ArH}\right), 7.26\left(\mathrm{t},{ }^{3} J_{\mathrm{HH}}=7.8 \mathrm{~Hz}, 1 \mathrm{H}, \mathrm{ArH}\right), 7.08$ (apt d, ${ }^{3} J_{\mathrm{HH}}=$ $7.7 \mathrm{~Hz}, 4 \mathrm{H}, \mathrm{ArH}), 6.85\left(\mathrm{~d},{ }^{3} J_{\mathrm{HH}}=8.2 \mathrm{~Hz}, 1 \mathrm{H}, \mathrm{ArH}\right), 2.39\left(\mathrm{dt},{ }^{2} J_{\mathrm{PH}}=11.7 \mathrm{~Hz},{ }^{3} J_{\mathrm{HH}}=\right.$ 11.7 Hz 3H, PCH; PCy $), 1.65$ (s, 14H, $\left.\mathrm{CH}_{2} ; \mathrm{PCy}_{3}\right), 1.38$ (s, 6 $\left.\mathrm{H} \mathrm{CH}_{2} ; \mathrm{PCy}_{3}\right), 1.16$ (s, $\left.10 \mathrm{H}, \mathrm{CH}_{2} ; \mathrm{PCy}_{3}\right) .{ }^{19} \mathrm{~F}\left\{{ }^{1} \mathrm{H}\right\} \mathrm{NMR}\left(470.67 \mathrm{MHz}, \mathrm{CD}_{2} \mathrm{Cl}_{2}\right): \delta=-55.72\left(\mathrm{~s}, \mathrm{NCF}_{3}\right)$. ${ }^{31} \mathrm{P}\left\{{ }^{1} \mathrm{H}\right\}$ NMR $\left(202.50 \mathrm{MHz}, \mathrm{CD}_{2} \mathrm{Cl}_{2}\right): \delta=30.11\left(\mathrm{~s}, \mathrm{PCy}_{3}\right) \cdot{ }^{13} \mathrm{C}\left\{{ }^{1} \mathrm{H}\right\}$ NMR $(125.81$ $\mathrm{MHz}, \mathrm{CD}_{2} \mathrm{Cl}_{2}$ ): $\delta=304.29\left(\mathrm{~d},{ }^{2} J_{\mathrm{PC}}=29.8 \mathrm{~Hz}, \mathrm{RuCHPh}\right), 207.54(\mathrm{dd}, J=85.1,3.4 \mathrm{~Hz}$, $\mathrm{NCN}), 138.30\left(\mathrm{C}_{\mathrm{Ar}}\right), 136.15\left(\mathrm{C}_{\mathrm{Ar}}\right), 131.76$ (br s, $\left.\mathrm{C}_{\mathrm{Ar}}\right), 131.11\left(\mathrm{~d}, J=2.5 \mathrm{~Hz}, \mathrm{CH}_{\mathrm{Ar}}\right)$, $130.32\left(\mathrm{CH}_{\mathrm{Ar}}\right), 130.24\left(\mathrm{CH}_{\mathrm{Ar}}\right), 129.31\left(\mathrm{C}_{\mathrm{Ar}}\right), 128.76\left(\mathrm{CH}_{\mathrm{Ar}}\right), 128.46\left(4 \mathrm{x} \mathrm{CH}_{\mathrm{Ar}}\right), 125.47$ $\left(\mathrm{CH}_{\mathrm{Ar}}\right), 125.15\left(\mathrm{CH}_{\mathrm{Ar}}\right), 121.67\left(\mathrm{q},{ }^{1} J_{\mathrm{CF}}=263.9 \mathrm{~Hz}, \mathrm{NCF}_{3}\right), 112.34\left(\mathrm{CH}_{\mathrm{Ar}}\right), 111.37$ $\left(\mathrm{CH}_{\mathrm{Ar}}\right), 32.45\left(\mathrm{~d},{ }^{1} J_{\mathrm{PC}}=16.5 \mathrm{~Hz}, \mathrm{PCH} ; \mathrm{PCy}_{3}\right), 29.28\left(\mathrm{CH}_{2} ; \mathrm{PCy}_{3}\right), 28.40\left(\mathrm{CH}_{2} ; \mathrm{PCy}_{3}\right)$, $28.32\left(\mathrm{CH}_{2} ; \mathrm{PCy}_{3}\right), 27.11\left(\mathrm{CH}_{2} ; \mathrm{PCy}\right) . \mathrm{IR}$ (neat): 3047.4, 2933.3, 2847.3, 1368.6, 
1320.1, 1172.8, 751.4 $\mathrm{cm}^{-1}$. HRMS (MALDI/ESI): $\left[\mathrm{M}^{+}-\mathrm{Cl}\right]$ calcd for $\mathrm{C}_{39} \mathrm{H}_{48}$ $\mathrm{ClN}_{2} \mathrm{~F}_{3} \mathrm{PRu}$ 769.22; found 769.2241. Mp: 137.1 - $138.1{ }^{\circ} \mathrm{C}$ with decomposition. Crystals suitable for X-ray diffraction analysis were obtained by slow diffusion of hexane into a concentrated solution of $\mathbf{6 a}$ in $\mathrm{CH}_{2} \mathrm{Cl}_{2}$. Despite multiple attempts, no pure elemental analysis could be obtained for $\mathbf{6 a}$

\subsubsection{Tricyclohexylphosphine[1-(trifluoromethyl)-3-(4-(trifluoromethyl)phe- nyl)-1H-benzo[d]imidazol-3-ylidene][benzylidene]ruthenium dichloride (6b)}

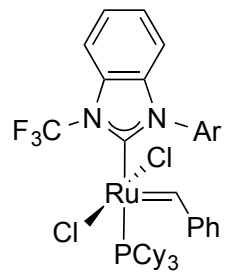

6b

Synthesized according to general procedure A from $\mathbf{5 b}$ (254 mg, 0.61 mmol), Grubbs $1^{\text {st }}$ generation catalyst $(500 \mathrm{mg}, 0.61 \mathrm{mmol})$. The product was obtained as a pale green solid $(316.8 \mathrm{mg}, 0.36 \mathrm{mmol}$, $60 \%$ ). ${ }^{1} \mathrm{H} \operatorname{NMR}\left(500.27 \mathrm{MHz}, \mathrm{CD}_{2} \mathrm{Cl}_{2}\right): \delta=19.23\left(\mathrm{~d},{ }^{3} J_{\mathrm{HH}}=4.9 \mathrm{~Hz}\right.$, 1H, RuCHPh), 7.91-7.37 (br s, 3H, ArH), 7.73 (d, ${ }^{3} J_{\mathrm{HH}}=8.3 \mathrm{~Hz}, 1 \mathrm{H}$, $\mathrm{Ar}=4-\mathrm{CF}_{3}-\mathrm{Ph}$ $\mathrm{ArH}), 7.45\left(\mathrm{dt},{ }^{3} J_{\mathrm{HH}}=12.1,{ }^{3} J_{\mathrm{HH}}=7.6 \mathrm{~Hz}, 2 \mathrm{H}, \mathrm{ArH}\right), 7.37-6.70$ (br s, $3 \mathrm{H}, \mathrm{ArH}), 7.31\left(\mathrm{t},{ }^{3} J_{\mathrm{HH}}=7.8 \mathrm{~Hz}, 1 \mathrm{H}, \mathrm{ArH}\right), 7.13\left(\mathrm{t},{ }^{3} J_{\mathrm{HH}}=7.6 \mathrm{~Hz}, 2 \mathrm{H}, \mathrm{ArH}\right), 6.88(\mathrm{~d}$, $\left.{ }^{3} J_{\mathrm{HH}}=8.2 \mathrm{~Hz}, 1 \mathrm{H}, \mathrm{ArH}\right), 2.42\left(\mathrm{dt},{ }^{2} J_{\mathrm{PH}}=12.0 \mathrm{~Hz},{ }^{3} J_{\mathrm{HH}}=12.0 \mathrm{~Hz}, 3 \mathrm{H}, \mathrm{PCH} ; \mathrm{PCy}_{3}\right)$, 1.65 (br s, $14 \mathrm{H}, \mathrm{CH}_{2} ; \mathrm{PCy}_{3}$ ), 1.40 (br s, $6 \mathrm{H}, \mathrm{CH}_{2} ; \mathrm{PCy}_{3}$ ), 1.19 (br s, $10 \mathrm{H}, \mathrm{CH}_{2} ; \mathrm{PCy}_{3}$ ). ${ }^{19} \mathrm{~F}\left\{{ }^{1} \mathrm{H}\right\}$ NMR (470.67 MHz, $\left.\mathrm{CD}_{2} \mathrm{Cl}_{2}\right): \delta=-55.69\left(\mathrm{~s}, \mathrm{NCF}_{3}\right),-63.07\left(\mathrm{~s}, \mathrm{ArCF}_{3}\right)$. ${ }^{31} \mathrm{P}\left\{{ }^{1} \mathrm{H}\right\}$ NMR $\left(202.50 \mathrm{MHz}, \mathrm{CD}_{2} \mathrm{Cl}_{2}\right): \delta=29.98(\mathrm{~s}, \mathrm{PCy}) .{ }^{13} \mathrm{C}\left\{{ }^{1} \mathrm{H}\right\}$ NMR $(125.81$ $\mathrm{MHz}, \mathrm{CD}_{2} \mathrm{Cl}_{2}$ ): $\delta=304.86$ (apt d, ${ }^{2} J_{\mathrm{PC}}=29.4 \mathrm{~Hz}, \mathrm{RuCHPh}$ ), 207.73 (apt dd, $J=85.6$, $2.4 \mathrm{~Hz}, \mathrm{NCN}), 151.14\left(\mathrm{C}_{\mathrm{Ar}}\right), 139.21\left(\mathrm{C}_{\mathrm{Ar}}\right), 137.74$ (apt d, $J=2.4 \mathrm{~Hz}, \mathrm{C}_{\mathrm{Ar}}$ ), 131.85 (q, $J$ $\left.=32.7 \mathrm{~Hz}, \mathrm{C}_{\mathrm{Ar}}\right), 131.20\left(\mathrm{~d}, J=2.4 \mathrm{~Hz}, \mathrm{C}_{\mathrm{Ar}}\right), 130.78\left(\mathrm{CH}_{\mathrm{Ar}}\right), 129.42\left(\mathrm{br} \mathrm{s}, 4 \mathrm{x} \mathrm{CH}_{\mathrm{Ar}}\right)$, 128.90 (br s, 2x CH $\mathrm{CH}_{\mathrm{Ar}}$ ), 126.49 (br s, 2x $\left.\mathrm{CH}_{\mathrm{Ar}}\right), 125.73\left(\mathrm{CH}_{\mathrm{Ar}}\right), 125.54\left(\mathrm{CH}_{\mathrm{Ar}}\right), 124.11$ $\left(\mathrm{q},{ }^{1} J_{\mathrm{CF}}=272.6 \mathrm{~Hz}, \mathrm{ArCF}_{3}\right), 121.59\left(\mathrm{q},{ }^{1} J_{\mathrm{CF}}=264.4 \mathrm{~Hz}, \mathrm{NCF}_{3}\right), 112.00\left(\mathrm{CH}_{\mathrm{Ar}}\right), 111.63$ $\left(\mathrm{CH}_{\mathrm{Ar}}\right), 32.48\left(\mathrm{~d},{ }^{1} J_{P C}=16.7 \mathrm{~Hz}, \mathrm{PCH} ; \mathrm{PCy}_{3}\right), 29.30\left(\mathrm{CH}_{2} ; \mathrm{PCy}_{3}\right), 28.39\left(\mathrm{CH}_{2} ; \mathrm{PCy}_{3}\right)$, $28.31\left(\mathrm{CH}_{2} ; \mathrm{PCy}_{3}\right), 27.10\left(\mathrm{CH}_{2} ; \mathrm{PCy}_{3}\right)$. IR (neat): 2921.7, 2849.7, 1360.7, 1319.3, 1186.6, 1127.9, 1025.5, $748.7 \mathrm{~cm}^{-1}$. HRMS (MALDI/ESI): $\left[\mathrm{M}^{+}-\mathrm{Cl}\right]$ calcd for $\mathrm{C}_{40} \mathrm{H}_{47} \mathrm{ClN}_{2} \mathrm{~F}_{6} \mathrm{PRu}$ 837.21; found 837.2102. Anal. Calcd (\%) for $\mathrm{C}_{40} \mathrm{H}_{47} \mathrm{Cl}_{2} \mathrm{~N}_{2} \mathrm{~F}_{6} \mathrm{PRu}: \mathrm{C}$, 55.05; H, 5.43; N, 3.21. Found: C, 54.86; H, 5.63; N, 3.07. Mp: $166.5-167.5^{\circ} \mathrm{C}$ with decomposition. Crystals suitable for X-ray diffraction analysis were obtained by slow diffusion of hexane into a concentrated solution of $\mathbf{6} \mathbf{b}$ in $\mathrm{CH}_{2} \mathrm{Cl}_{2}$. 
2.5.3 Tricyclohexylphosphine[3-(3,5-bis(trifluoromethyl)phenyl)-1(trifluoromethyl)-1H-benzo[d]imidazol-3-ylidene][benzylidene]ruthenium dichloride (6c).

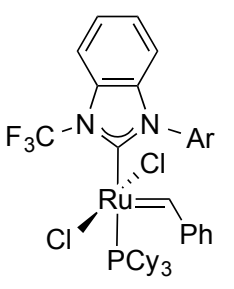

6c

$\mathrm{Ar}=3,5-\mathrm{CF}_{3}-\mathrm{Ph}$

Synthesized according to general procedure A from 5c $(236 \mathrm{mg}$, $0.49 \mathrm{mmol}$ ), Grubbs $1^{\text {st }}$ generation catalyst (400 mg, $0.49 \mathrm{mmol}$ ).

The product was obtained as a yellow green solid $(152.1 \mathrm{mg}, 0.16$ mmol, 33\%). ${ }^{1} \mathrm{H}$ NMR (500.27 MHz, $\left.\mathrm{CD}_{2} \mathrm{Cl}_{2}\right): \delta=19.23\left(\mathrm{~d},{ }^{3} J_{H H}=\right.$ $7.2 \mathrm{~Hz}, 1 \mathrm{H}, \mathrm{RuCHPh}), 8.68$ (br s, 1H, ArH), 8.22 (br s, 1H, ArH),

$7.77\left(\mathrm{~d},{ }^{3} J_{\mathrm{HH}}=8.2 \mathrm{~Hz}, 1 \mathrm{H}, \mathrm{ArH}\right), 7.65(\mathrm{~s}, 1 \mathrm{H}, \mathrm{ArH}), 7.48\left(\mathrm{dd},{ }^{3} J_{\mathrm{HH}}\right.$ $\left.=7.6 \mathrm{~Hz},{ }^{3} J_{\mathrm{HH}}=7.6 \mathrm{~Hz}, 2 \mathrm{H}, \mathrm{ArH}\right), 7.43($ br s, $1 \mathrm{H}, \mathrm{ArH}), 7.36\left(\mathrm{t},{ }^{3} J_{\mathrm{HH}}=7.8 \mathrm{~Hz}, 1 \mathrm{H}\right.$, ArH), $7.22-6.89$ (br s, 1H, ArH), 7.12 (apt t, $\left.{ }^{3} J_{\mathrm{HH}}=7.0 \mathrm{~Hz}, 2 \mathrm{H}, \mathrm{ArH}\right), 6.96\left(\mathrm{~d},{ }^{3} J_{\mathrm{HH}}=\right.$ $8.2 \mathrm{~Hz}, 1 \mathrm{H}, \mathrm{ArH}), 2.45\left(\mathrm{dt},{ }^{2} J_{\mathrm{PH}}=11.9 \mathrm{~Hz},{ }^{3} J_{\mathrm{HH}}=11.9 \mathrm{~Hz}, 3 \mathrm{H}, \mathrm{PCH} ; \mathrm{PCy}_{3}\right), 1.79$ (br s, 6H, $\mathrm{CH}_{2} ; \mathrm{PCy}_{3}$ ), 1.66 (br s, 9H, $\mathrm{CH}_{2} ; \mathrm{PCy}_{3}$ ), 1.43 (br s, 6H, $\mathrm{CH}_{2} ; \mathrm{PCy}_{3}$ ), 1.21 (br s, 9H, $\left.\mathrm{CH}_{2} ; \mathrm{PCy}_{3}\right) .{ }^{19} \mathrm{~F}\left\{{ }^{1} \mathrm{H}\right\} \mathrm{NMR}\left(470.67 \mathrm{MHz}, \mathrm{CD}_{2} \mathrm{Cl}_{2}\right): \delta=-55.38\left(\mathrm{~s}, \mathrm{NCF}_{3}\right),-62.83$ (br s, $\mathrm{ArCF}_{3}$ ), -63.07 (br s, $\left.\mathrm{ArCF}_{3}\right) .{ }^{31} \mathrm{P}\left\{{ }^{1} \mathrm{H}\right\} \mathrm{NMR}\left(202.50 \mathrm{MHz}, \mathrm{CD}_{2} \mathrm{Cl}_{2}\right): \delta=28.17$ (s, $\left.\mathrm{PCy}_{3}\right) .{ }^{13} \mathrm{C}\left\{{ }^{1} \mathrm{H}\right\}$ NMR $\left(125.81 \mathrm{MHz}, \mathrm{CD}_{2} \mathrm{Cl}_{2}\right): \delta=306.86\left(\mathrm{~d},{ }^{2} J_{\mathrm{CP}}=29.0 \mathrm{~Hz}\right.$, $\mathrm{RuCHPh}), 207.83$ (d, $J=89.8 \mathrm{~Hz}, \mathrm{NCN}), 150.22\left(\mathrm{C}_{\mathrm{Ar}}\right), 138.02\left(\mathrm{C}_{\mathrm{Ar}}\right), 136.83$ (apt d, $J=$ $2.5 \mathrm{~Hz}, 2 \mathrm{x} \mathrm{C}_{\mathrm{Ar}}$ ), 132.54 (br s, $\mathrm{CH}_{\mathrm{Ar}}$ ), $131.69\left(\mathrm{CH}_{\mathrm{Ar}}\right), 131.41$ (apt d, $J=2.4 \mathrm{~Hz}, 2 \mathrm{x} \mathrm{C} \mathrm{Cr}_{\mathrm{Ar}}$ ), 130.53 (br s, $\left.\mathrm{CH}_{\mathrm{Ar}}\right), 129.51-128.19$ (br m, 4x CH $\left.\mathrm{CH}_{\mathrm{Ar}}\right), 126.06\left(\mathrm{CH}_{\mathrm{Ar}}\right), 126.00\left(\mathrm{CH}_{\mathrm{Ar}}\right)$, 123.96 (apt p, $J=3.8 \mathrm{~Hz}, \mathrm{CH}_{\mathrm{Ar}}$ ), 123.12 (C-F HMQC, ${ }^{1} J_{\mathrm{CF}}=276.96, \mathrm{ArCF}_{3}$ ), 123.12 $\left(\mathrm{C}-\mathrm{F}\right.$ HMQC, $\left.{ }^{1} J_{\mathrm{CF}}=276.21 \mathrm{~Hz}, \mathrm{ArCF}_{3}\right), 121.59\left(\mathrm{q},{ }^{1} J_{\mathrm{CF}}=264.8 \mathrm{~Hz}, \mathrm{NCF}_{3}\right), 112.06$ $\left(\mathrm{CH}_{\mathrm{Ar}}\right), 111.62\left(\mathrm{CH}_{\mathrm{Ar}}\right), 32.53\left(\mathrm{~d},{ }^{1} J_{\mathrm{PC}}=16.4 \mathrm{~Hz}, \mathrm{PCH} ; \mathrm{PCy}_{3}\right), 29.44$ (apt d, $J=48.6$ $\left.\mathrm{Hz}, \mathrm{CH}_{2} ; \mathrm{PCy}_{3}\right), 28.34\left(\mathrm{CH}_{2} ; \mathrm{PCy}_{3}\right), 28.26\left(\mathrm{CH}_{2} ; \mathrm{PCy}\right), 27.11\left(\mathrm{CH}_{2} ; \mathrm{PCy}_{3}\right)$. IR (neat): 2931.7, 2848.51, 1364.5, 1320.7, 1274.2, 1187.0 (br), 1137.9 (br), 1081.5 (br), 1037.8, $765.4 \mathrm{~cm}^{-1}$. HRMS (MALDI/ESI): $\left[\mathrm{M}^{+}-\mathrm{Cl}\right]$ calcd for $\mathrm{C}_{41} \mathrm{H}_{46} \mathrm{ClN}_{2} \mathrm{~F}_{9} \mathrm{PRu}$ 905.20; found 905.1985. Anal. Calcd (\%) for $\mathrm{C}_{41} \mathrm{H}_{46} \mathrm{Cl}_{2} \mathrm{~N}_{2} \mathrm{~F}_{9} \mathrm{PRu}$ : C, 52.35; H, 4.93; N, 2.98. Found: C, 52.53; H, 5.08; N, 3.03. Mp: $154.6-155.2{ }^{\circ} \mathrm{C}$ with decomposition.

2.5.4 Tricyclohexylphosphine[3-butyl-1-(trifluoromethyl)-1Hbenzo $[d]$ imidazol-3-ylidene][benzylidene]ruthenium dichloride (6d).

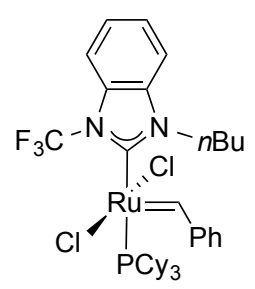

6d

Synthesized according to general procedure A from 5d (239 mg, 0.61 mmol), Grubbs $1^{\text {st }}$ generation catalyst $(500 \mathrm{mg}, 0.61 \mathrm{mmol})$. The product was obtained as a pale green solid $(386 \mathrm{mg}, 0.25 \mathrm{mmol}$, 41\%). ${ }^{1} \mathrm{H} \mathrm{NMR}\left(500.27 \mathrm{MHz}, \mathrm{CD}_{2} \mathrm{Cl}_{2}\right): \delta=20.46\left(\mathrm{~d},{ }^{3} J_{\mathrm{HH}}=4.1 \mathrm{~Hz}\right.$, 
1H, RuCHPh), 8.56 (br s, 2H, ArH), 7.66 (s, 2H, ArH), $7.41-7.30$ (m, 5H, ArH), 3.67 (br s, $\left.2 \mathrm{H}, \mathrm{NCH}_{2}\left(\mathrm{CH}_{2}\right)_{2} \mathrm{CH}_{3}\right), 2.45\left(\mathrm{dt},{ }^{2} J_{\mathrm{PH}}=11.9 \mathrm{~Hz},{ }^{3} J_{\mathrm{HH}}=11.9 \mathrm{~Hz} 3 \mathrm{H}, \mathrm{PCH} ; \mathrm{PCy}_{3}\right)$, 1.76 - 1.67 (br m, 14H, $\mathrm{CH}_{2} ; \mathrm{PCy}_{3}$ ), 1.46 - 1.37 (br m, 8H, $\mathrm{CH}_{2} ; \mathrm{PCy}_{3}$ ), 1.20 (br s, $10 \mathrm{H}, \mathrm{CH}_{2} ; \mathrm{PCy}_{3}$ ), 0.77 (br s, $2 \mathrm{H}, \mathrm{CH}_{2} \mathrm{CH}_{2} \mathrm{CH}_{3}$ ), 0.66 (apt t, ${ }^{2} J_{\mathrm{HH}}=6.8 \mathrm{~Hz}, 3 \mathrm{H}, \mathrm{CH}_{3}$ ). ${ }^{19} \mathrm{~F}\left\{{ }^{1} \mathrm{H}\right\}$ NMR $\left(470.67 \mathrm{MHz}, \mathrm{CD}_{2} \mathrm{Cl}_{2}\right): \delta=-55.00\left(\mathrm{~s}, \mathrm{NCF}_{3}\right),{ }^{31} \mathrm{P}\left\{{ }^{1} \mathrm{H}\right\} \mathrm{NMR}(202.50$ $\left.\mathrm{MHz}, \mathrm{CD}_{2} \mathrm{Cl}_{2}\right): \delta=31.80\left(\mathrm{~s}, \mathrm{PCy}_{3}\right) .{ }^{13} \mathrm{C}\left\{{ }^{1} \mathrm{H}\right\} \mathrm{NMR}\left(125.81 \mathrm{MHz}, \mathrm{CD}_{2} \mathrm{Cl}_{2}\right): \delta=301.44$ (RuCHPh), 204.46 (apt dt, $J=82.5,3.0 \mathrm{~Hz}, \mathrm{NCN}), 153.30\left(\mathrm{C}_{\mathrm{Ar}}\right), 136.19$ (d, $J=2.0 \mathrm{~Hz}$, $\left.\mathrm{C}_{\mathrm{Ar}}\right), 131.79\left(\mathrm{CH}_{\mathrm{Ar}}\right), 131.77\left(\mathrm{CH}_{\mathrm{Ar}}\right), 130.82\left(\mathrm{CH}_{\mathrm{Ar}}\right), 129.81\left(2 \mathrm{x} \mathrm{CH}_{\mathrm{Ar}}\right), 129.22\left(\mathrm{C}_{\mathrm{Ar}}\right)$, $125.31\left(\mathrm{CH}_{\mathrm{Ar}}\right), 124.91\left(\mathrm{CH}_{\mathrm{Ar}}\right), 121.53\left(\mathrm{q},{ }^{1} J_{\mathrm{CF}}=263.4 \mathrm{~Hz}, \mathrm{NCF}_{3}\right), 111.83\left(2 \mathrm{x} \mathrm{CH}_{\mathrm{Ar}}\right)$, $48.43\left(\mathrm{CH}_{2}\right), 32.69\left(\mathrm{~d},{ }^{1} J_{\mathrm{PC}}=16.0 \mathrm{~Hz}, \mathrm{PCH} ; \mathrm{PCy}\right), 31.58\left(\mathrm{NCH}_{2}\left(\mathrm{CH}_{2}\right)_{2} \mathrm{CH}_{3}\right), 29.28$ $\left(\mathrm{CH}_{2} ; \mathrm{PCy}_{3}\right), 28.40\left(\mathrm{CH}_{2} ; \mathrm{PCy}\right), 28.32\left(\mathrm{CH}_{2} ; \mathrm{PCy}\right), 27.09\left(\mathrm{CH}_{2} ; \mathrm{PCy}_{3}\right), 20.03$ $\left(\mathrm{CH}_{2} \mathrm{CH}_{2} \mathrm{CH}_{3}\right), 13.93\left(\mathrm{CH}_{3}\right)$. IR (neat): 2926.50, 2849.75, 1400.69, 1357.32, 1232.14,

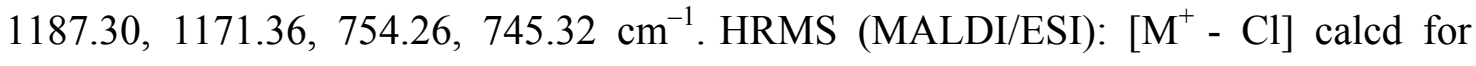
$\mathrm{C}_{37} \mathrm{H}_{52} \mathrm{ClN}_{2} \mathrm{~F}_{3} \mathrm{PRu}$ 749.26; found 749.2553. Anal. Calcd (\%) for $\mathrm{C}_{37} \mathrm{H}_{52} \mathrm{Cl}_{2} \mathrm{~N}_{2} \mathrm{~F}_{3} \mathrm{PRu}: \mathrm{C}$, 56.63; H, 6.68; N, 3.57. Found: C, 55.26; H, 6.67; N, 3.53 (probably, solvent impurities accounted for the deviation in carbon content). Mp: $145.1-146.1{ }^{\circ} \mathrm{C}$ with decomposition. Crystals suitable for X-ray diffraction analysis were obtained by slow diffusion of hexane into a concentrated solution of $\mathbf{6} \mathbf{d}$ in $\mathrm{CH}_{2} \mathrm{Cl}_{2}$.

\subsubsection{Tricyclohexylphosphine[1-isopropyl-3-(4-(trifluoromethyl)phenyl)-1H- benzo $[d]$ imidazol-3-ylidene][benzylidene]ruthenium dichloride (7)}

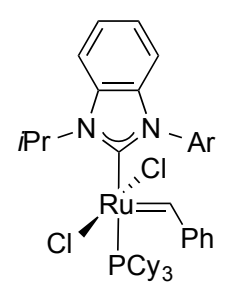

7

Synthesized according to general procedure A from D $(238 \mathrm{mg}, 0.61$ mmol), Grubbs $1^{\text {st }}$ generation catalyst $(500 \mathrm{mg}, 0.61 \mathrm{mmol})$. The product was obtained as a red-brown solid (320 $\mathrm{mg}, 0.38 \mathrm{mmol}, 62 \%)$. ${ }^{1} \mathrm{H}$ NMR $\left(500.27 \mathrm{MHz}, \mathrm{CD}_{2} \mathrm{Cl}_{2}\right): \delta=19.22\left(\mathrm{~d},{ }^{3} J_{\mathrm{HH}}=3.8 \mathrm{~Hz}, 1 \mathrm{H}\right.$, RuCHPh), $8.17-7.56$ (br m, 2H, ArH), 7.68 (d, ${ }^{3} J_{\mathrm{HH}}=8.2 \mathrm{~Hz}, 1 \mathrm{H}$, ArH), $7.42\left(\mathrm{t},{ }^{3} J_{\mathrm{HH}}=7.3 \mathrm{~Hz}, 1 \mathrm{H}, \mathrm{ArH}\right), 7.29-7.07$ (m, 4H, ArH), 7.42 $\left(\mathrm{t},{ }^{3} J_{\mathrm{HH}}=7.3 \mathrm{~Hz}, 1 \mathrm{H}, \mathrm{ArH}\right), 7.29-7.26(\mathrm{~m}, 2 \mathrm{H}, \mathrm{ArH}), 7.14-7.07$ (m, 2H, ArH), 6.84 $\left(\mathrm{d},{ }^{3} J_{\mathrm{HH}}=8.1 \mathrm{~Hz}, 1 \mathrm{H}, \mathrm{ArH}\right), 5.59\left(\mathrm{p},{ }^{3} J_{\mathrm{HH}}=6.7 \mathrm{~Hz}, 1 \mathrm{H}, \mathrm{NCH}\left(\mathrm{CH}_{3}\right)_{2}\right), 2.45\left(\mathrm{dt},{ }^{2} J_{\mathrm{PH}}=\right.$ $\left.11.8 \mathrm{~Hz},{ }^{3} J_{\mathrm{HH}}=11.8 \mathrm{~Hz}, 3 \mathrm{H}, \mathrm{PCH}\right), 1.99\left(\mathrm{~d},{ }^{3} J_{\mathrm{HH}}=6.8 \mathrm{~Hz}, 6 \mathrm{H}, \mathrm{CH}_{3}\right), 1.75-1.69$ (br m, 14H, $\mathrm{CH}_{2} ; \mathrm{PCy}_{3}$ ), 1.43 - 1.36 (br m, 6H, $\mathrm{CH}_{2} ; \mathrm{PCy}_{3}$ ), 1.17 (br s, $10 \mathrm{H}, \mathrm{CH}_{2} ; \mathrm{PCy}_{3}$ ). ${ }^{19} \mathrm{~F}\left\{{ }^{1} \mathrm{H}\right\}$ NMR (470.67 MHz, $\left.\mathrm{CD}_{2} \mathrm{Cl}_{2}\right): \delta=-62.91\left(\mathrm{~s}, \mathrm{ArCF}_{3}\right) .{ }^{31} \mathrm{P}\left\{{ }^{1} \mathrm{H}\right\} \mathrm{NMR}(202.50$ $\left.\mathrm{MHz}, \mathrm{CD}_{2} \mathrm{Cl}_{2}\right): \delta=34.54\left(\mathrm{~s}, \mathrm{PCy}_{3}\right) .{ }^{13} \mathrm{C}\left\{{ }^{1} \mathrm{H}\right\} \mathrm{NMR}\left(125.81 \mathrm{MHz}, \mathrm{CD}_{2} \mathrm{Cl}_{2}\right): \delta=299.77$ (dd, $\left.{ }^{2} J_{P C}=37.4, J=8.0 \mathrm{~Hz}, \mathrm{RuCHPh}\right), 199.99$ (d, $\left.J=81.6 \mathrm{~Hz}, \mathrm{NCN}\right), 151.15\left(\mathrm{C}_{\mathrm{Ar}}\right)$, 
$140.46\left(\mathrm{C}_{\mathrm{Ar}}\right), 138.72\left(\mathrm{C}_{\mathrm{Ar}}\right), 132.86\left(\mathrm{~d}, J=3.1 \mathrm{~Hz}, \mathrm{C}_{\mathrm{Ar}}\right), 131.04$ (apt q, $J=32.4 \mathrm{~Hz}$, $\left.\mathrm{CH}_{\mathrm{Ar}}\right), 130.89$ (br s, 2x CH $\left.\mathrm{CH}_{\mathrm{Ar}}\right), 129.93\left(\mathrm{CH}_{\mathrm{Ar}}\right), 129.77\left(\mathrm{CH}_{\mathrm{Ar}}\right), 126.27\left(4 \mathrm{x} \mathrm{CH}_{\mathrm{Ar}}\right)$, $124.26\left(\mathrm{q},{ }^{1} J_{\mathrm{CF}}=274.5 \mathrm{~Hz}, \mathrm{ArCF}_{3}\right), 123.39\left(\mathrm{CH}_{\mathrm{Ar}}\right), 123.16\left(\mathrm{CH}_{\mathrm{Ar}}\right), 112.45\left(\mathrm{CH}_{\mathrm{Ar}}\right)$, $111.44\left(\mathrm{CH}_{\mathrm{Ar}}\right), 55.61\left(\mathrm{NCH}\left(\mathrm{CH}_{3}\right)_{2}\right), 32.26\left(\mathrm{~d},{ }^{1} J_{\mathrm{PC}}=16.5 \mathrm{~Hz}, \mathrm{PCH}\right), 29.99\left(\mathrm{CH}_{2}\right.$; $\left.\mathrm{PCy}_{3}\right), 28.40\left(\mathrm{CH}_{2} ; \mathrm{PCy}_{3}\right), 28.32\left(\mathrm{CH}_{2} ; \mathrm{PCy}_{3}\right), 27.31\left(\mathrm{CH}_{2} ; \mathrm{PCy}_{3}\right), 21.22\left(2 \mathrm{x} \mathrm{CH}_{3}\right) . \mathrm{IR}$ (neat) $2916.3,2848.4,1385.1,1318.0,1161.3,1123.4,1065.7,741.2 \mathrm{~cm}^{-1}$. HRMS (MALDI/ESI): [ $\mathrm{M}^{+}-\mathrm{Cl}$ ] calcd for $\mathrm{C}_{42} \mathrm{H}_{54} \mathrm{ClN}_{2} \mathrm{~F}_{3} \mathrm{PRu}$ 811.27; found 811.2711. Anal. Calcd (\%) for $\mathrm{C}_{42} \mathrm{H}_{54} \mathrm{Cl}_{2} \mathrm{~N}_{2} \mathrm{~F}_{3} \mathrm{PRu}$ : C, 59.57; H, 6.43; N, 3.31. Found: C, 59.96; H, 6.74; $\mathrm{N}, 3.07$. Mp: $187.8-188.8^{\circ} \mathrm{C}$ with decomposition.

\subsection{General procedure B: Ring Closing Metathesis of Diethyl Diallylmalonate}

In a glove box, a Young NMR tube is charged with a solution of the catalyst $(0.6 \mu \mathrm{mol}$, $1 \mathrm{~mol} \%$ ) in $0.6 \mathrm{~mL}$ of $\mathrm{CD}_{2} \mathrm{Cl}_{2}$. The Young NMR tube is then equilibrated at $30{ }^{\circ} \mathrm{C}$ in the NMR probe. Subsequently, diethyl diallylmalonate (14.6 $\mu \mathrm{L}, 0.06 \mathrm{mmol}, 1$ equiv) was injected into the tube and the reaction was monitored over time by ${ }^{1} \mathrm{H}-\mathrm{NMR}$ spectroscopy.

\subsection{General procedure C: Cross Metathesis of allyl benzene with cis-1,4- diacetoxy-2-butene}

In a glove box, a $8 \mathrm{~mL}$ vial was charged with the catalyst (0.02 mmol, $2.5 \mathrm{~mol} \%$ ) and $3.0 \mathrm{ml}$ of toluene and placed in an orbital shaker preheated to $40{ }^{\circ} \mathrm{C}$. Subsequently, $1.0 \mathrm{ml}$ of a toluene solution containing allylbenzene (108 $\mu \mathrm{L}, 0.8 \mathrm{mmol}, 1$ equiv), cis1,4-diacetoxybutene ( $263 \mu \mathrm{L}, 1.6 \mathrm{mmol}, 2$ equiv) and $n$-tridecane ( $99 \mu \mathrm{L}, 0.4 \mathrm{mmol}$, 0.5 equiv) was added and the reaction mixture sealed and agitated at $300 \mathrm{rpm}$. Aliquots $(30 \mu \mathrm{L})$ were dispensed into GC vials at specified time periods, quenched with $500 \mu \mathrm{L}$ of a $3 \mathrm{M}$ ethyl vinyl ether solution in toluene, crimp sealed and then analyzed by gas chromatography to determine conversion and selectivity (Figures S1S6 and Table S1). 


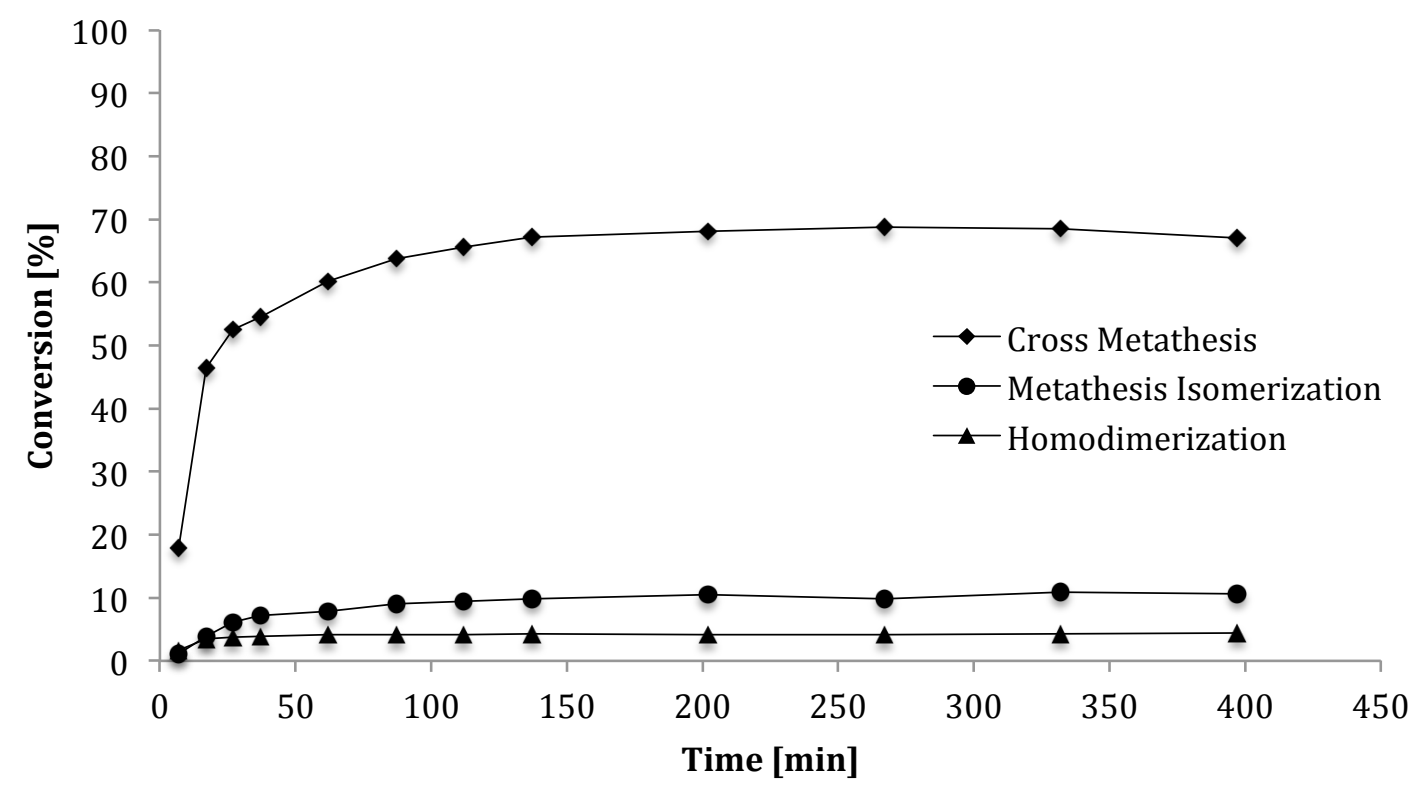

Figure S1. Conversion and selectivity of catalyst $\mathbf{6 a}$ in cross metathesis of allyl benzene with cis-1,4-diacetoxy-2-butene.

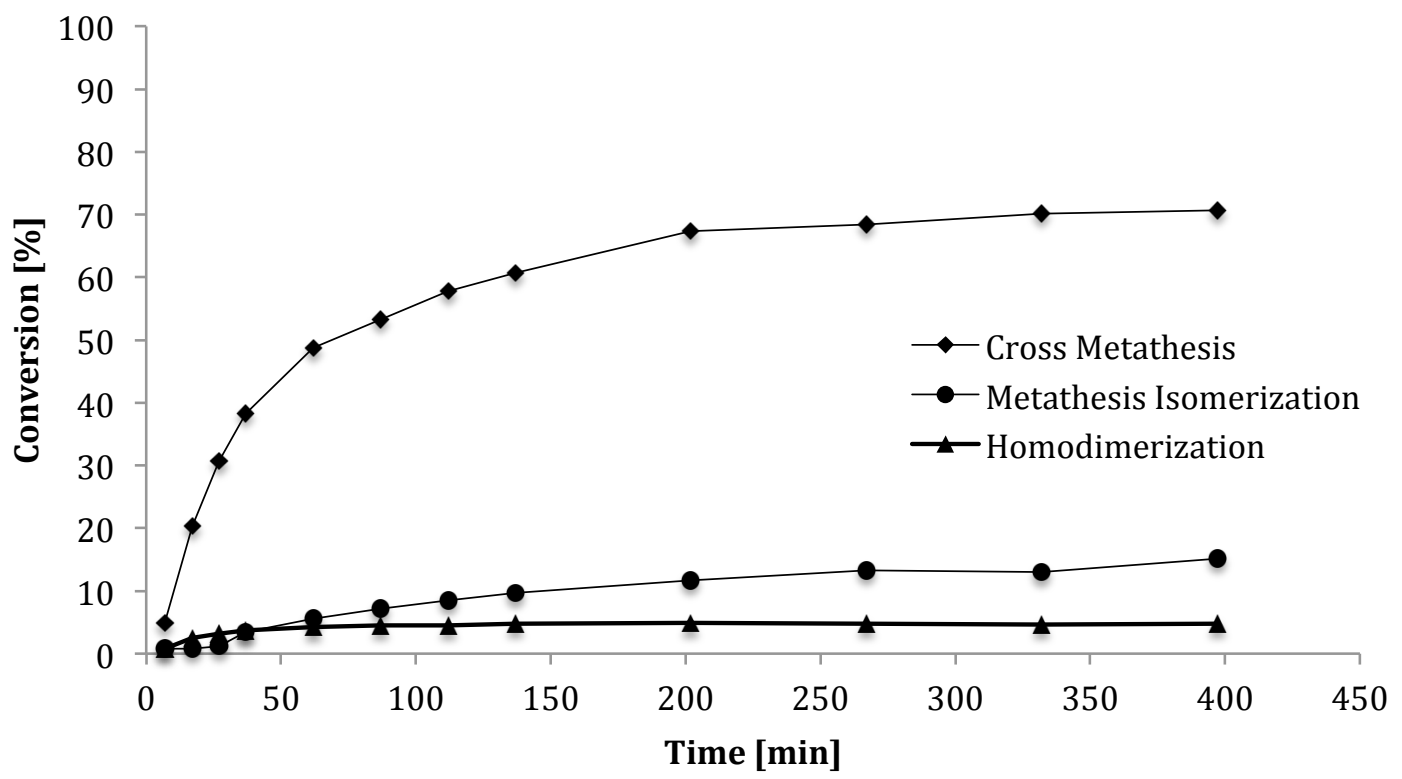

Figure S2. Conversion and selectivity of catalyst $\mathbf{6 b}$ in cross metathesis of allyl benzene with cis-1,4-diacetoxy-2-butene. 


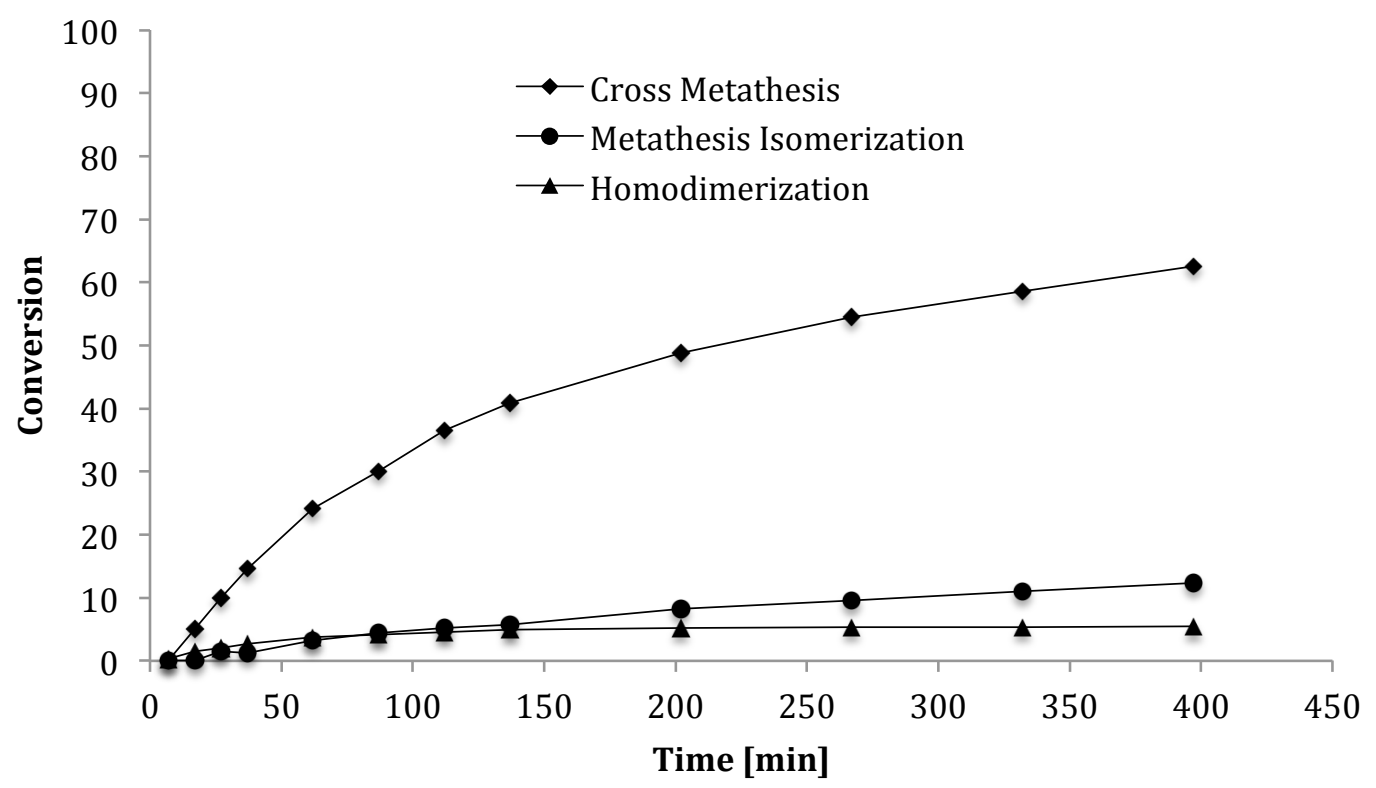

Figure S3. Conversion and selectivity of catalyst $\mathbf{6 c}$ in cross metathesis of allyl benzene with cis-1,4-diacetoxy-2-butene.

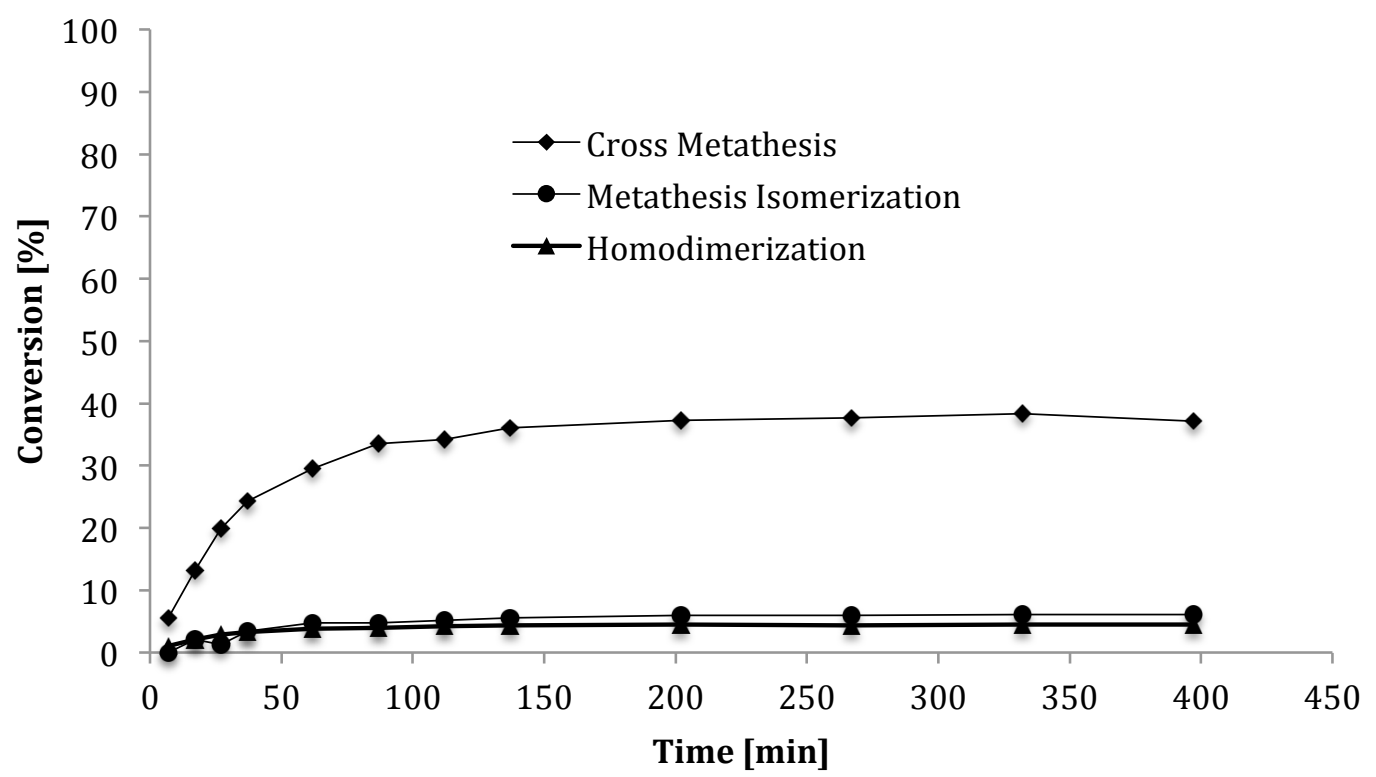

Figure S4. Conversion and selectivity of catalyst $\mathbf{6 d}$ in cross metathesis of allyl benzene with cis-1,4-diacetoxy-2-butene. 


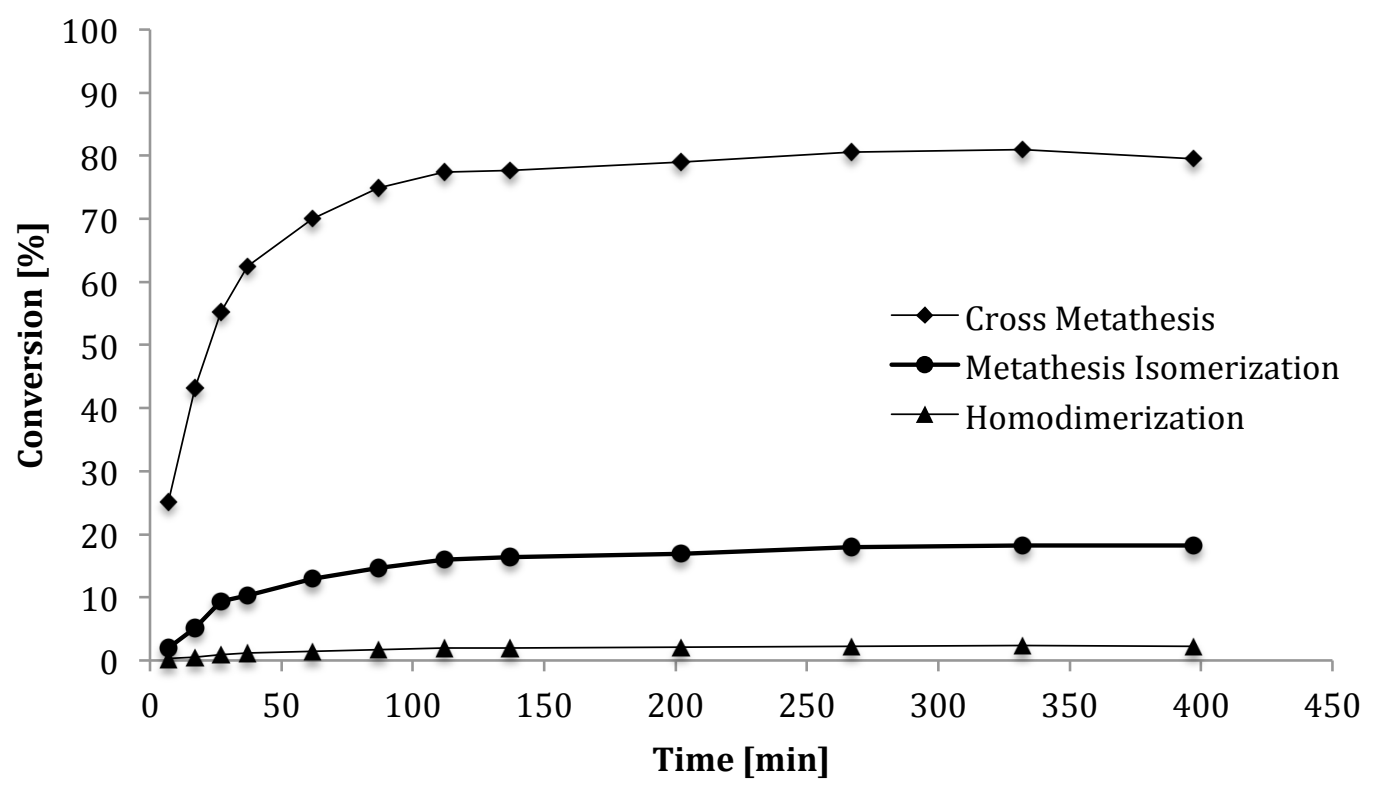

Figure S5. Conversion and selectivity of catalyst 7 in cross metathesis of allyl benzene with cis-1,4-diacetoxy-2-butene. 
Table S1. Conversion and selectivity displayed by catalysts $\mathbf{6 a}-\mathbf{d}$ and $\mathbf{7}$ in the end of the reaction.

\begin{tabular}{cccccc}
\hline entry & {$[\mathrm{Ru}]$} & $\begin{array}{c}\text { Cross } \\
\text { Metathesis [\%] }\end{array}$ & $\begin{array}{c}\text { E/Z ratio of } \\
\text { Cross Metathesis }\end{array}$ & $\begin{array}{c}\text { Metathesis } \\
\text { Isomerization } \\
{[\%]}\end{array}$ & Homodimerization [\%] \\
\hline 1 & $\mathbf{6 a}$ & 67 & 5.4 & 11 & 4 \\
2 & $\mathbf{6 b}$ & 71 & 5.4 & 15 & 5 \\
3 & $\mathbf{6 c}$ & 63 & 4.6 & 12 & 5 \\
4 & $\mathbf{6 d}$ & 37 & 3.9 & 6 & 2 \\
5 & 7 & 79 & 6.7 & 18 & 2 \\
\hline
\end{tabular}

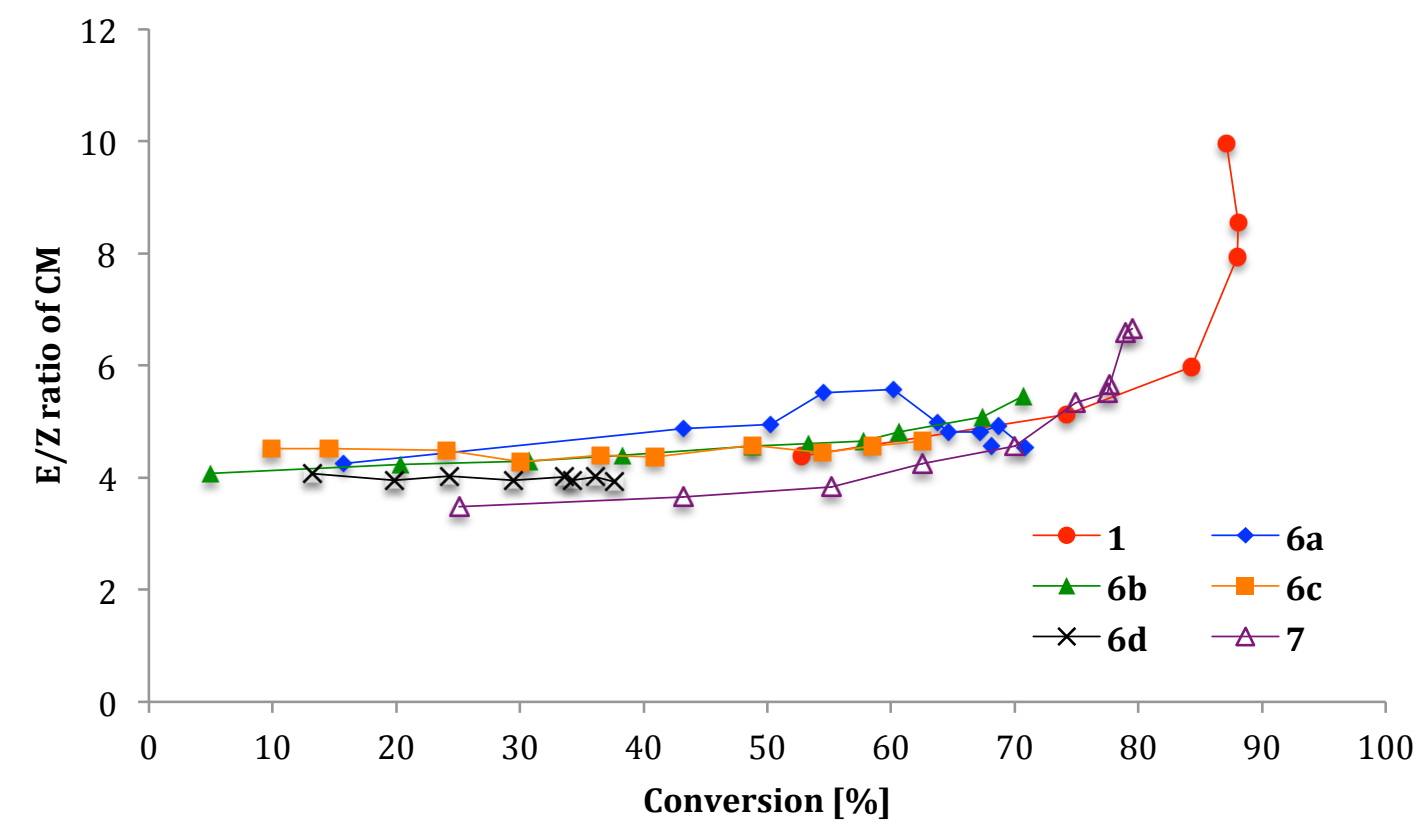

Figure S6. E/Z selectivity for the cross metathesis of allyl benzene with cis-1,4diacetoxy-2-butene with catalysts $\mathbf{1}, \mathbf{6 a -} \mathbf{d}$ and $\mathbf{7}$ as a function of the conversion. 


\subsection{General procedure D: Copolymerization of Norbornene and Cis- Cyclooctene. ${ }^{13} \mathrm{C}$ NMR spectra of polymers.}

Norbornene (40 mg, $0.42 \mathrm{mmol}, 1$ equiv) and cyclooctene $(2310 \mathrm{mg}, 21 \mathrm{mmol}, 50$ equiv) were dissolved in $\mathrm{CH}_{2} \mathrm{Cl}_{2}(7 \mathrm{~mL})$. Then $100 \mu \mathrm{L}$ of a $2.1 \cdot 10^{-3} \mathrm{M}$ solution of the catalyst in $\mathrm{CH}_{2} \mathrm{Cl}_{2}$ was injected via syringe and the reaction was stirred at $30{ }^{\circ} \mathrm{C}$ for the specified amount of time. The reaction was then stopped by the addition of 100 $\mu \mathrm{L}$ of a $3 \mathrm{M}$ solution of ethyl vinyl ether in $\mathrm{CH}_{2} \mathrm{Cl}_{2}$. The polymer was precipitated in $60 \mathrm{~mL}$ of methanol and dried in vacuo. The ${ }^{1} \mathrm{H}$ - and ${ }^{13} \mathrm{C}-\mathrm{NMR}$ spectra for the copolymer containing ca. 98\% alternating diads are shown in Figure S7. ${ }^{13} \mathrm{C}$ NMR spectra were recorded from a saturated solution in $\mathrm{CDCl}_{3}$ applying 2048 to 4096 scans. The transversal relaxation time $t_{2}$ was set 5 seconds to allow reliable integration of the characteristic olefinic ${ }^{13} \mathrm{C}$ NMR signals.

Cis- and trans- poly(COE-alt-NBE) ${ }_{n}^{1} \mathrm{H}$ NMR $\left(400.13 \mathrm{MHz}, \mathrm{CD}_{3} \mathrm{Cl}\right): \delta=5.37-$ 5.27 (m, 4H, CH), 2.78 (br s, 1H, $\mathrm{CH}_{2}$ ), 2.46 (br s, 1H, $\left.\mathrm{CH}_{2}\right), 2.03-1.77$ (m, 7H, $\mathrm{CH}_{2}$ ), 1.30 (br s, 10H, CH ), 1.03 (apt q, ${ }^{3} J_{\mathrm{HH}}=13.4,{ }^{3} J_{\mathrm{HH}}=12.2 \mathrm{~Hz}, 1 \mathrm{H}, \mathrm{CH}_{2}$ ). ${ }^{13} \mathrm{C}\left\{{ }^{1} \mathrm{H}\right\}$ NMR $\left(100.62 \mathrm{MHz}, \mathrm{CD}_{3} \mathrm{Cl}\right): \delta=135.35(\mathrm{CH}), 135.22(\mathrm{CH}), 135.06(\mathrm{CH})$, $134.92(\mathrm{CH}), 128.79-128.63(\mathrm{~m}, \mathrm{CH}), 43.68\left(\mathrm{CH}_{2}\right), 42.32\left(\mathrm{CH}_{2}\right), 41.91\left(\mathrm{CH}_{2}\right), 38.43$ $\left(\mathrm{CH}_{2}\right), 38.18\left(\mathrm{CH}_{2}\right), 33.00\left(\mathrm{CH}_{2}\right), 32.83\left(\mathrm{CH}_{2}\right), 32.71\left(\mathrm{CH}_{2}\right), 32.53\left(\mathrm{CH}_{2}\right), 32.53$ $\left(\mathrm{CH}_{2}\right), 32.37\left(\mathrm{CH}_{2}\right), 30.09\left(\mathrm{CH}_{2}\right), 29.77\left(\mathrm{CH}_{2}\right), 29.34\left(\mathrm{CH}_{2}\right), 29.24\left(\mathrm{CH}_{2}\right), 27.67$ $\left(\mathrm{CH}_{2}\right)$. 


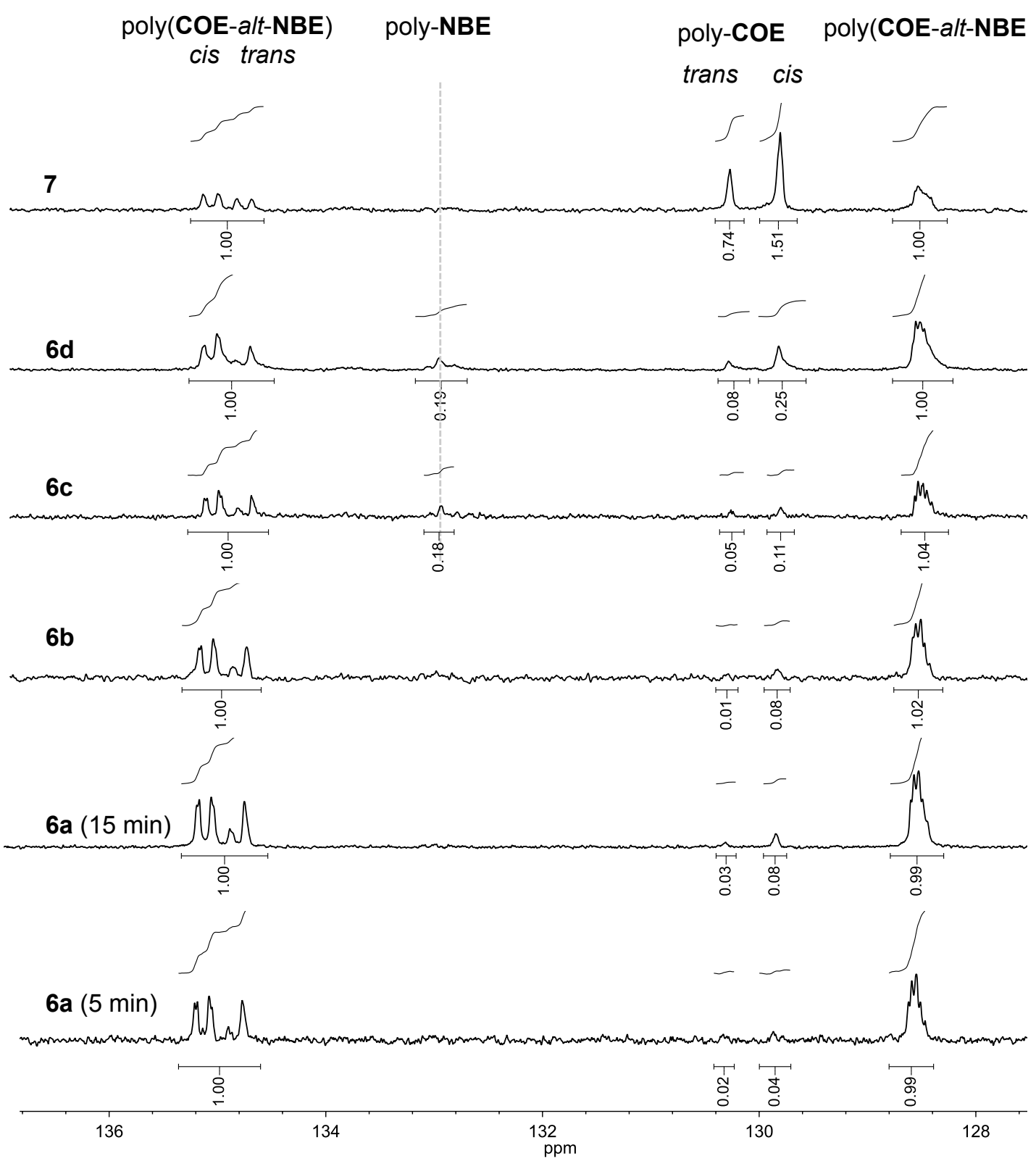

Figure S7. ${ }^{13} \mathrm{C}$ NMR spectra of poly(NBE-alt-COE) obtained with various catalysts 6-7. Due to signal-to-noise ratio, integrals of the minor signals were rounded upwards. 
Table S2. Selectivity of catalysts $\mathbf{6 b}$ and $\mathbf{7}$ in ROMP of COE.

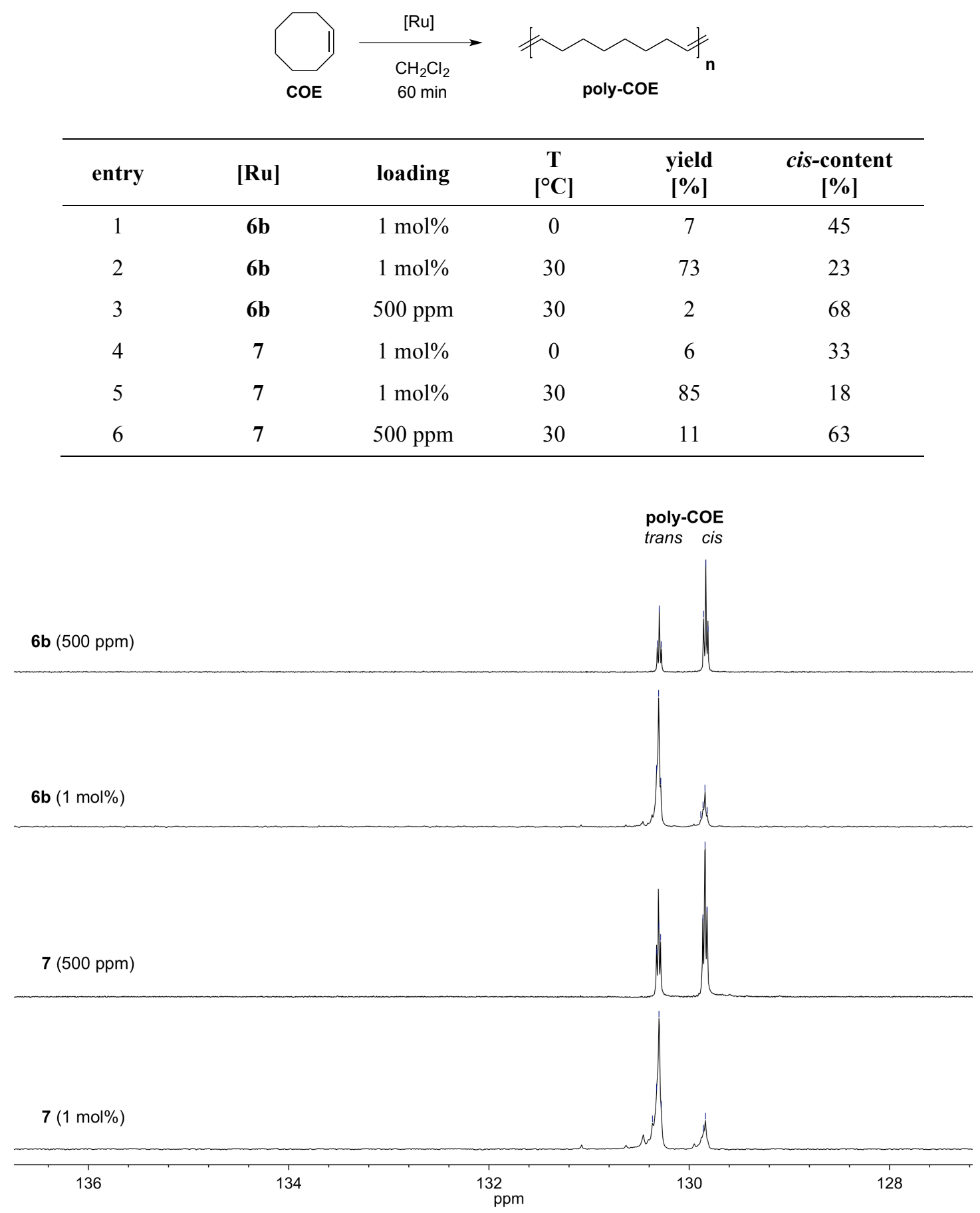

Figure S8. ${ }^{13} \mathrm{C}$ NMR spectra of poly-COE prepared with various loadings of $\mathbf{6 b}$ and 7 $\left(30^{\circ} \mathrm{C}\right)$. 


\subsection{General procedure E: Ethenolysis of Ethyl Oleate}

In a glove box, a reactor vial was charged with ethyl oleate $(1 \mathrm{~mL}, 2.802 \mathrm{mmol})$, purged 3 times with 20 bar of $\mathrm{C}_{2} \mathrm{H}_{4}$ and subsequently heated to $60{ }^{\circ} \mathrm{C}$ under 10 bar of $\mathrm{C}_{2} \mathrm{H}_{4}$. After $10 \mathrm{~min}$ at these conditions, a solution of the catalyst $\left(100 \mathrm{ppm}, 2.8 \cdot 10^{-4}\right.$ $\mathrm{mmol}$ ) in $0.25 \mathrm{ml}$ toluene was injected followed by an additional $0.75 \mathrm{ml}$ of toluene to wash the catalyst down the Endeavor parallel autoclave injecting system. The gas uptake was recorded at isobaric conditions (10 bar) for $60 \mathrm{~min}$ after addition of the last catalyst with $\sim 2$ min interval between each catalyst injection. After this time the reactors were cooled to $40{ }^{\circ} \mathrm{C}$ under ethylene, depressurized and the reaction mixtures quenched with activated $\mathrm{Al}_{2} \mathrm{O}_{3}$. Quantification by $\mathrm{GC}$ analysis gave conversions and allowed to calibrate back the $y$-axes on the gas uptake curves.

\subsection{General procedure F: Determination of activation parameters}

Inside a glove box, a volumetric flask was charged with the catalyst $(0.0353 \mathrm{mmol})$ and benzene- $\mathrm{d}_{6}$ (containing $0.005 \mathrm{M}$ ferrocene as an internal standard) was added to prepare $2.0 \mathrm{~mL}$ of stock solution $(0.0176 \mathrm{M})$. A Young NMR tube was charged with $0.6 \mathrm{~mL}(0.0106 \mathrm{mmol})$ of the stock solution. The sample was allowed to equilibrate in the NMR probe at the appropriate temperature. Subsequently, neat butyl vinyl ether (BVE) (41 $\mu \mathrm{L}, 0.318 \mathrm{mmol}, 30$ equiv relative to [Ru]) was injected into the Young NMR tube. All reactions were monitored by measuring the peak intensities of the starting alkylidene versus the peak intensity of ferrocene as a function of time. All reactions showed clean first-order kinetics over a period of at least three half-lives. 
<smiles></smiles><smiles>CC#CCCCCCC</smiles>

[Ru]<smiles></smiles><smiles>C=COCCCC</smiles><smiles></smiles>

$$
\begin{gathered}
\frac{-d[R u]}{d t}=k_{2}[R u][B V E]=\frac{k_{2} k_{1}[R u][B V E]}{k_{2}[B V E]+k_{-1}} \\
\text { for } k_{-1} \ll k_{2} \\
k_{o b s}=k_{1}[R u]=k_{1}
\end{gathered}
$$

Figure S9. Assumed dissociation mechanism for initiation of ruthenium catalysts $[\mathrm{Ru}]$.

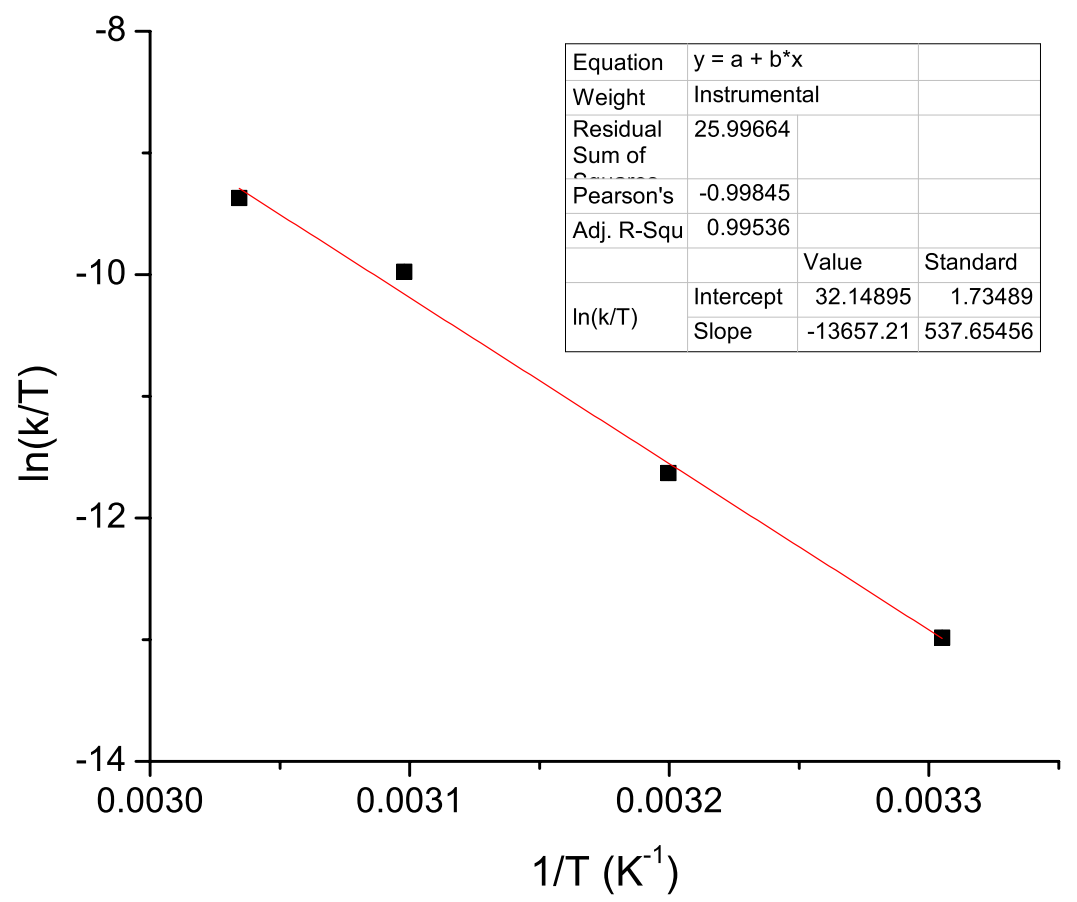

Figure S10. Eyring plot for reaction of $6 \mathbf{a}$ with BVE in benzene- $\mathrm{d}_{6}$. 


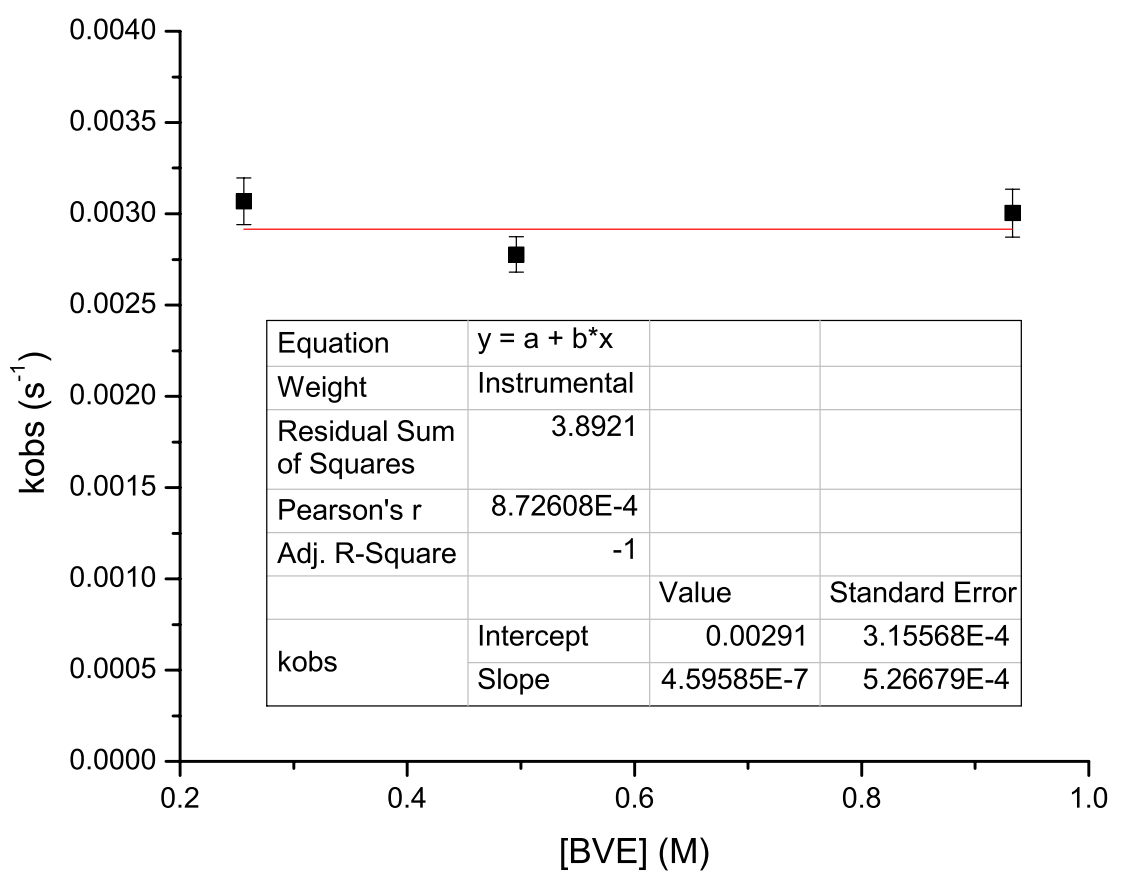

Figure S11. Dependence of the observed initiation rate constant $k_{\text {obs }}$ of $6 \mathbf{a}$ at $312.5 \mathrm{~K}$ on the butyl vinyl ether concentration [BVE].

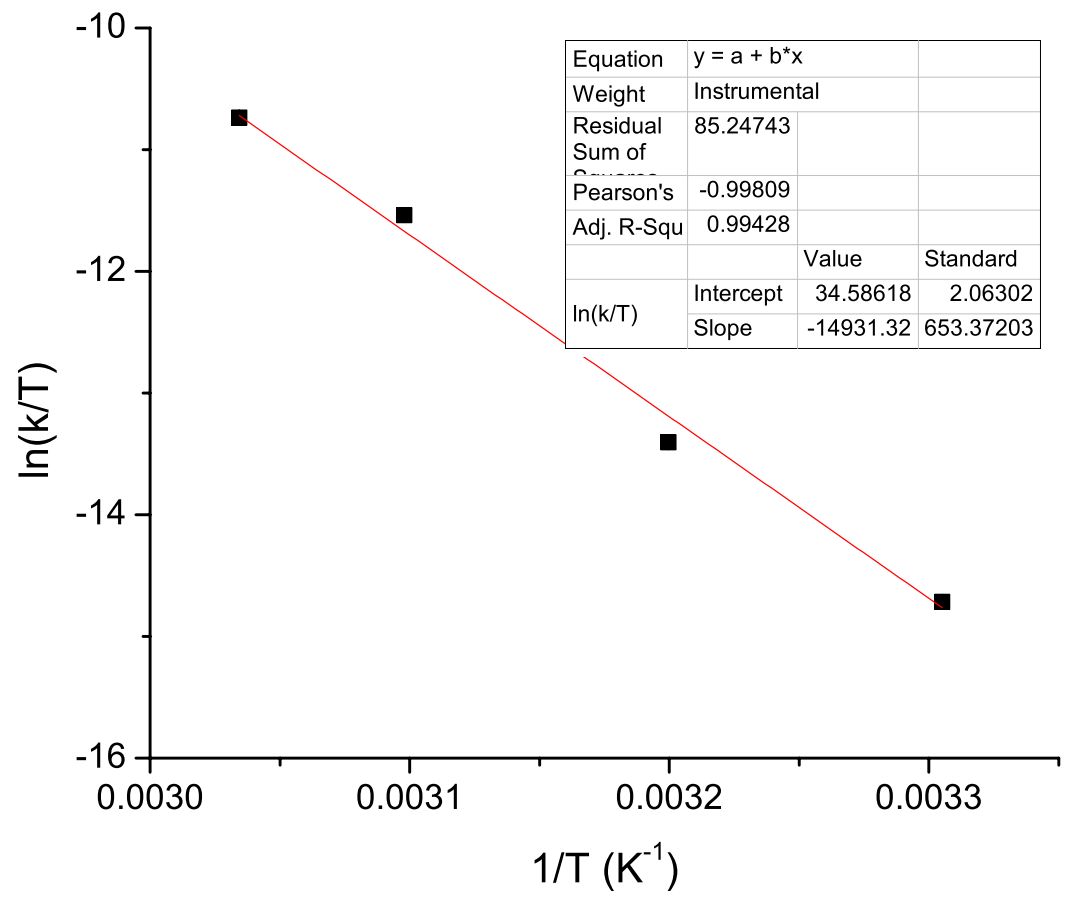

Figure S12. Eyring plot for reaction of $\mathbf{6 b}$ with BVE in benzene- $\mathrm{d}_{6}$. 


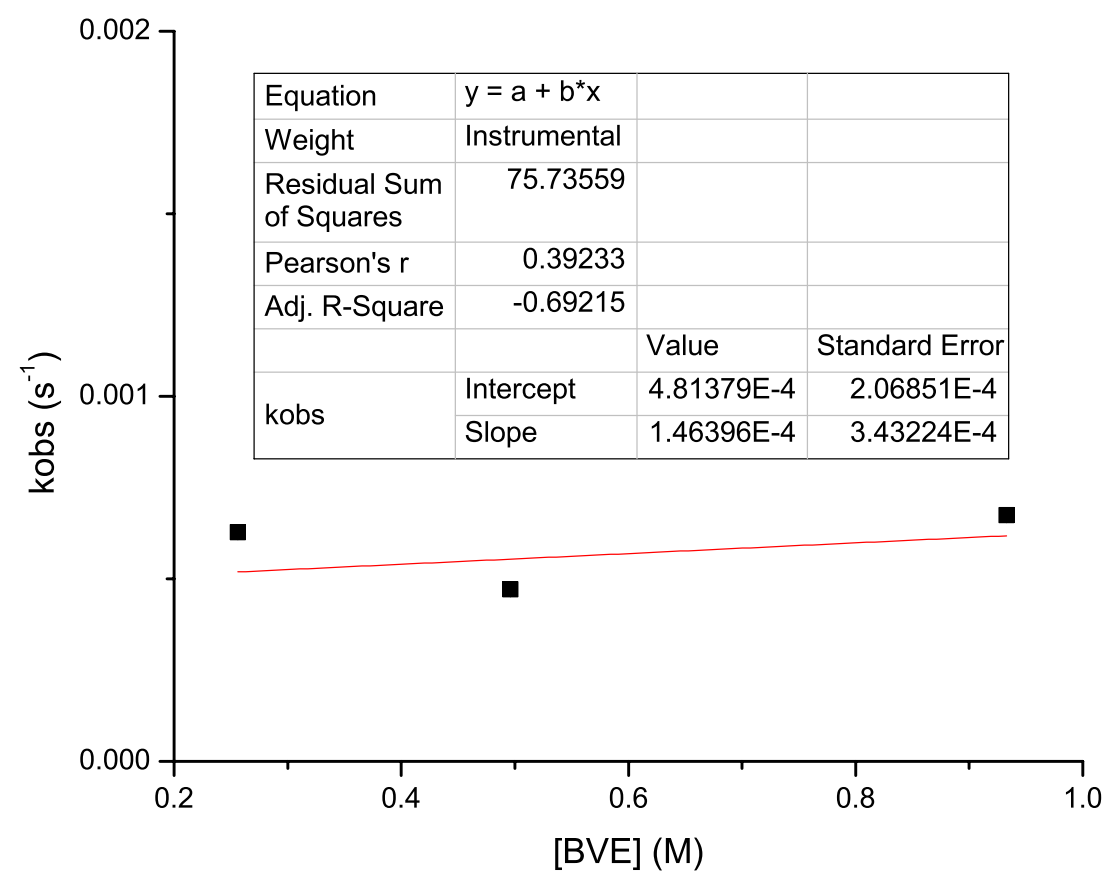

Figure S13. Dependence of the observed initiation rate constant $\mathrm{k}_{\mathrm{obs}}$ of $\mathbf{6 b}$ at $312.5 \mathrm{~K}$ on the butyl vinyl ether concentration [BVE].

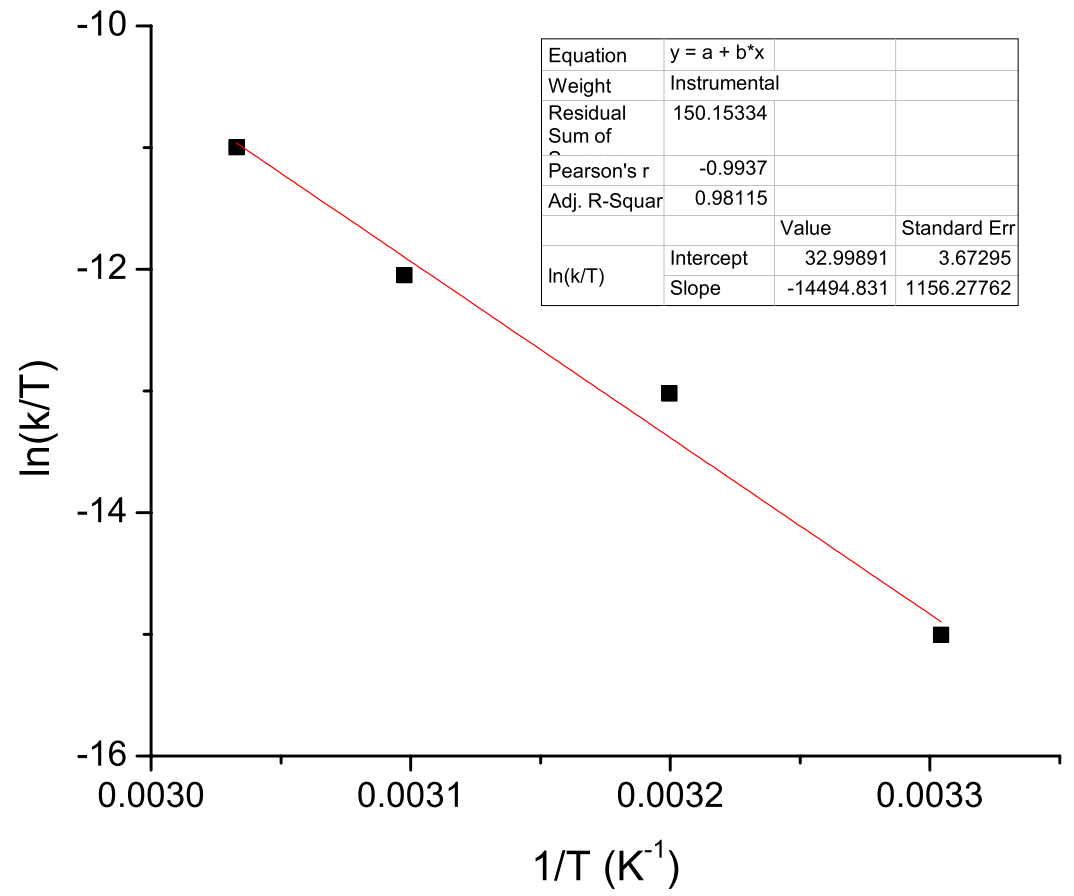

Figure S14. Eyring plot for reaction of $\mathbf{6 c}_{\mathbf{c}}$ with BVE in benzene-d $\mathrm{d}_{6}$. 


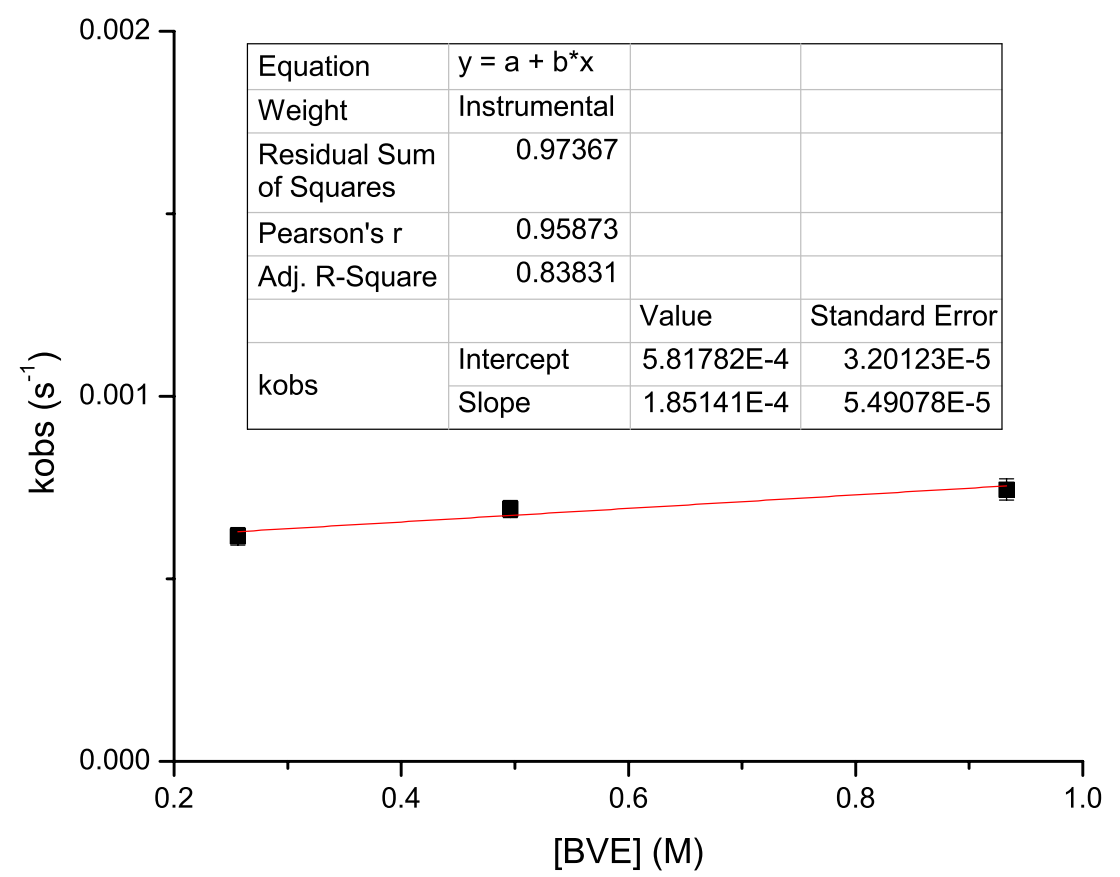

Figure S15. Dependence of the observed initiation rate constant $\mathrm{k}_{\mathrm{obs}}$ of $\mathbf{6 c}$ at $312.5 \mathrm{~K}$ on the butyl vinyl ether concentration [BVE].

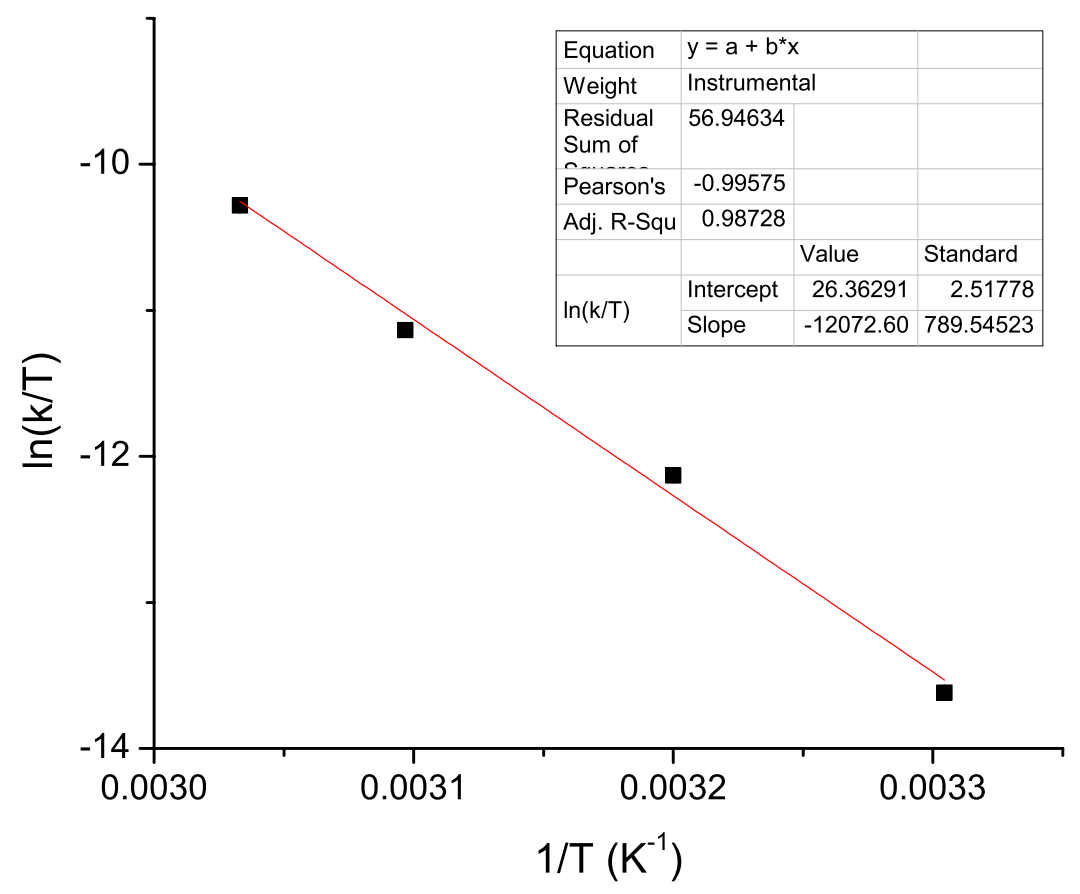

Figure S16. Eyring plot for reaction of $\mathbf{6} \mathbf{d}$ with BVE in benzene- $\mathrm{d}_{6}$ 


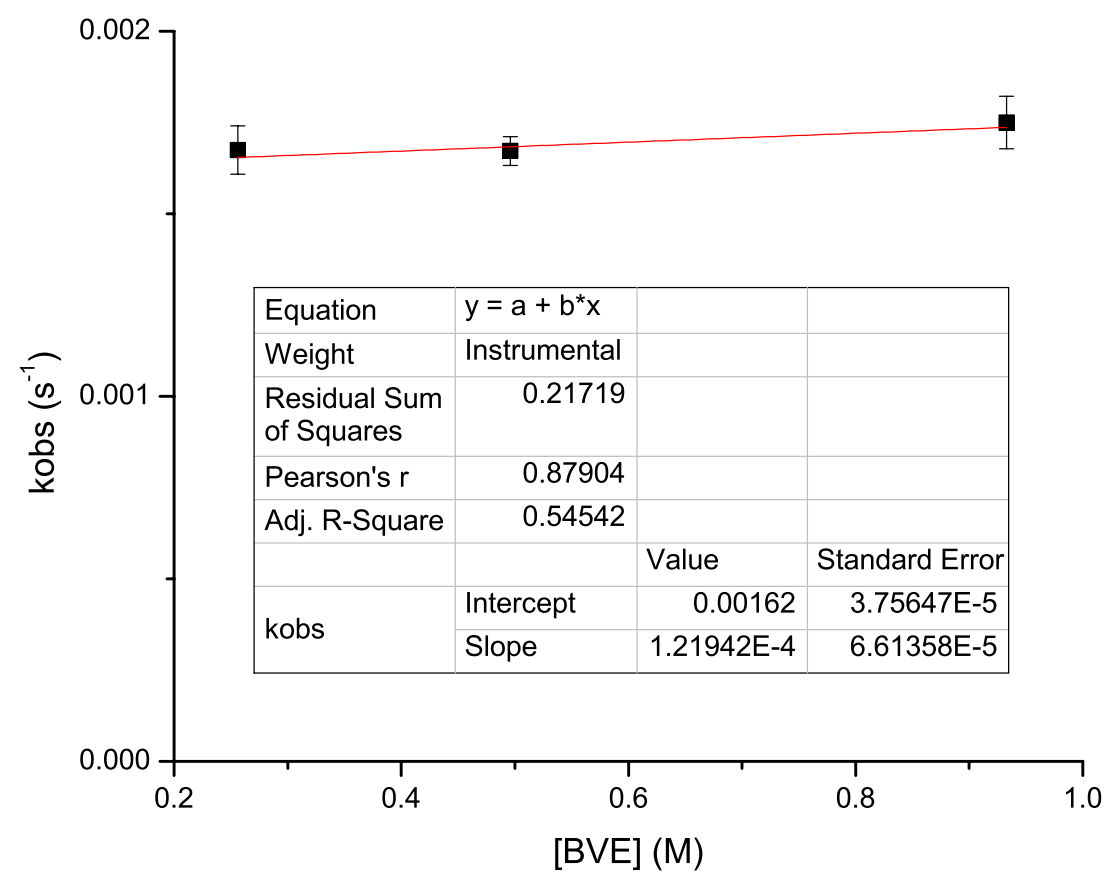

Figure S17. Dependence of the observed initiation rate constant $\mathrm{k}_{\mathrm{obs}}$ of $\mathbf{6 d}$ at $312.5 \mathrm{~K}$ on the butyl vinyl ether concentration [BVE].

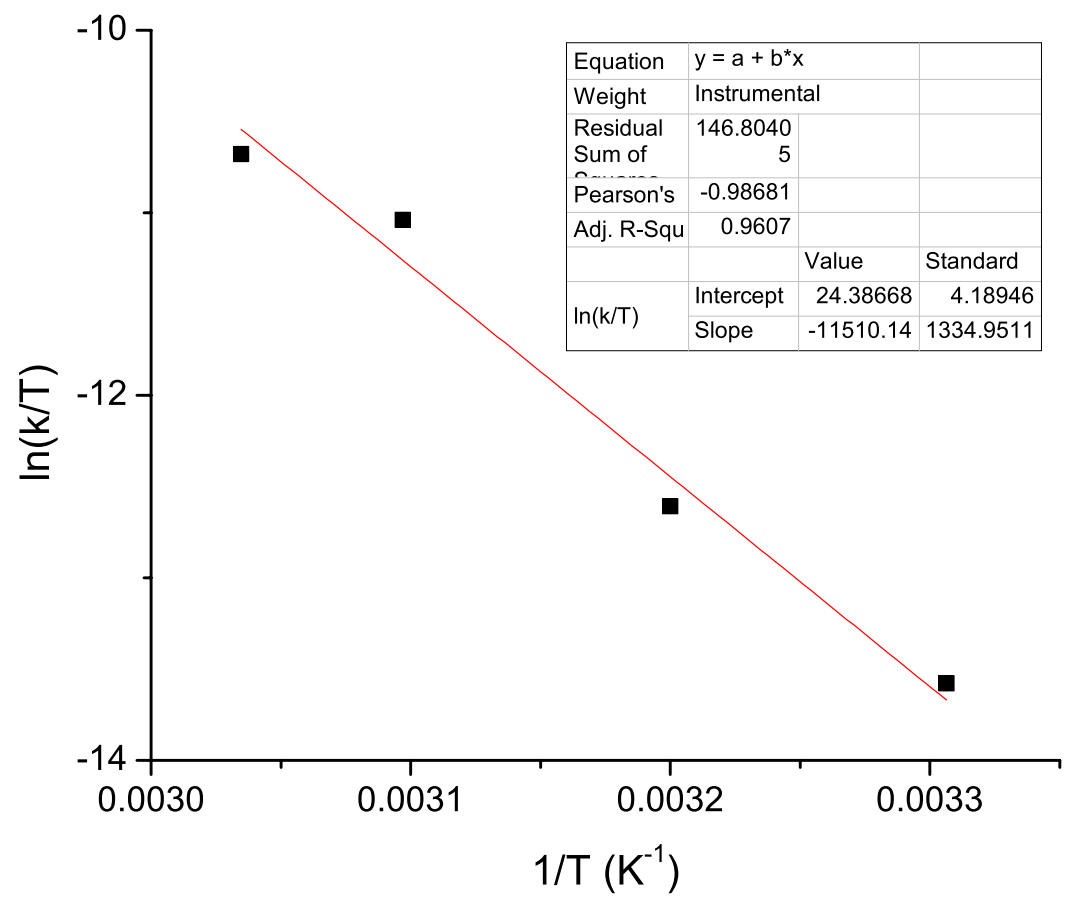

Figure S18. Eyring plot for reaction of 7 with BVE in benzene- $\mathrm{d}_{6}$ 


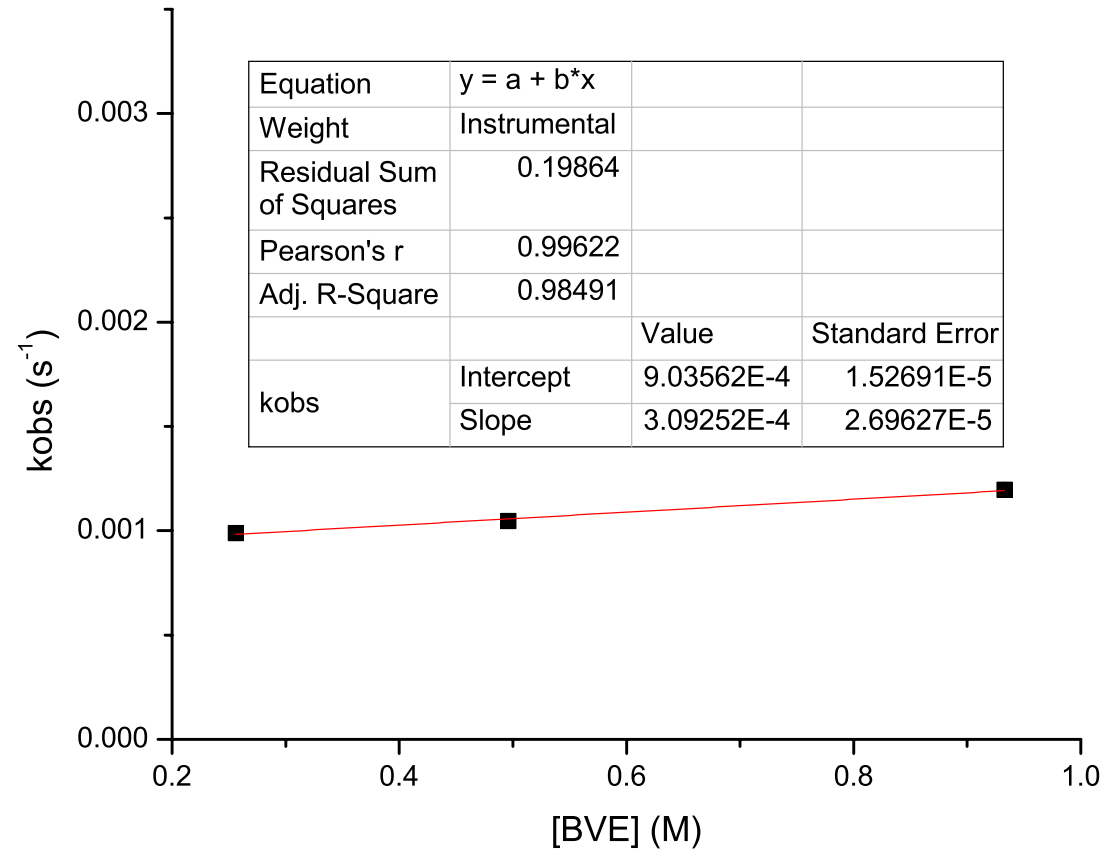

Figure S19. Dependence of the observed initiation rate constant $\mathrm{k}_{\mathrm{obs}}$ of 7 at $312.5 \mathrm{~K}$ on the butyl vinyl ether concentration [BVE]. 


\section{NOESY NMR Spectra of 6a-d}

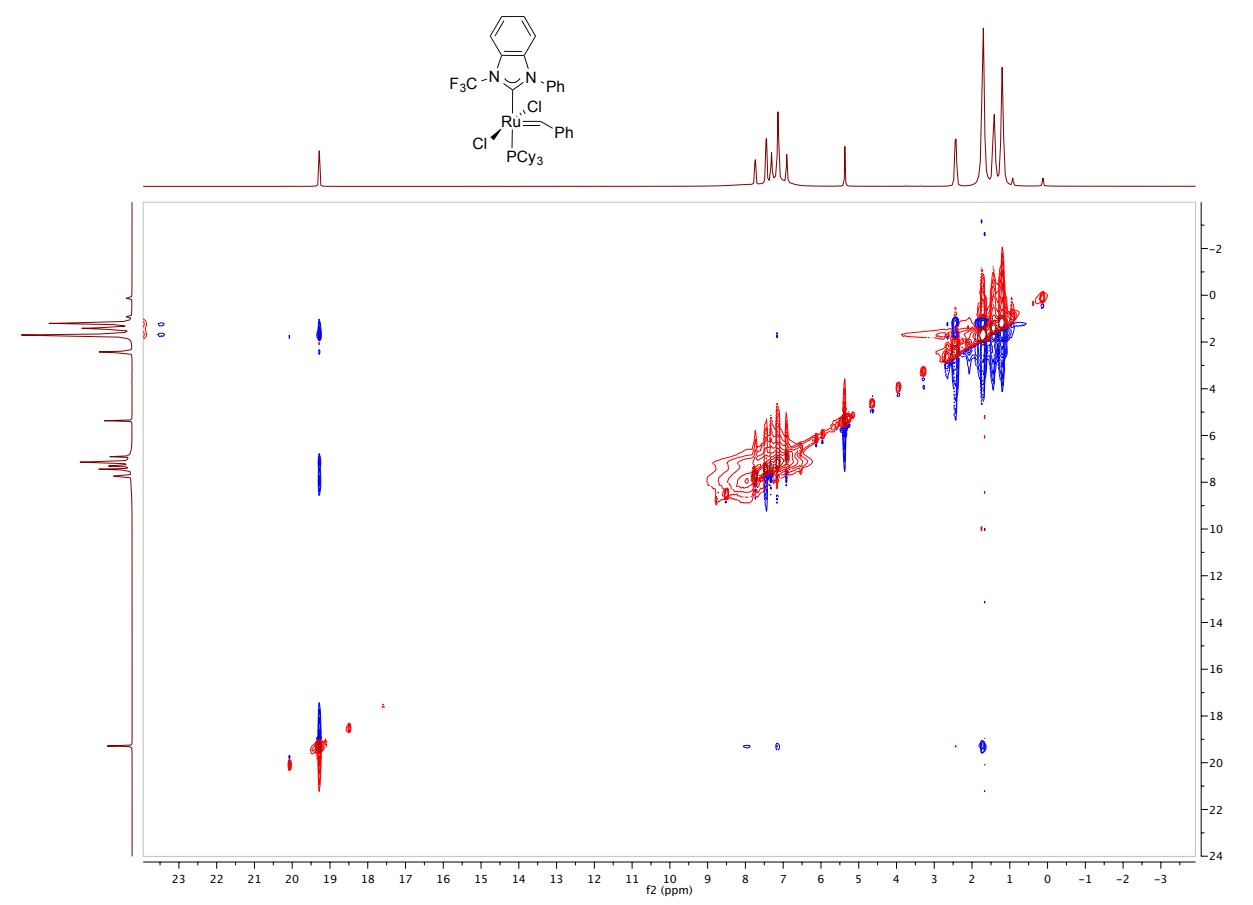

Figure S20. 2D ${ }^{1} \mathrm{H} /{ }^{1} \mathrm{H}$ NOESY NMR spectrum of 6a. Cross peaks between the benzylidene proton and the $\mathrm{N}$-aryl ortho-hydrogens.

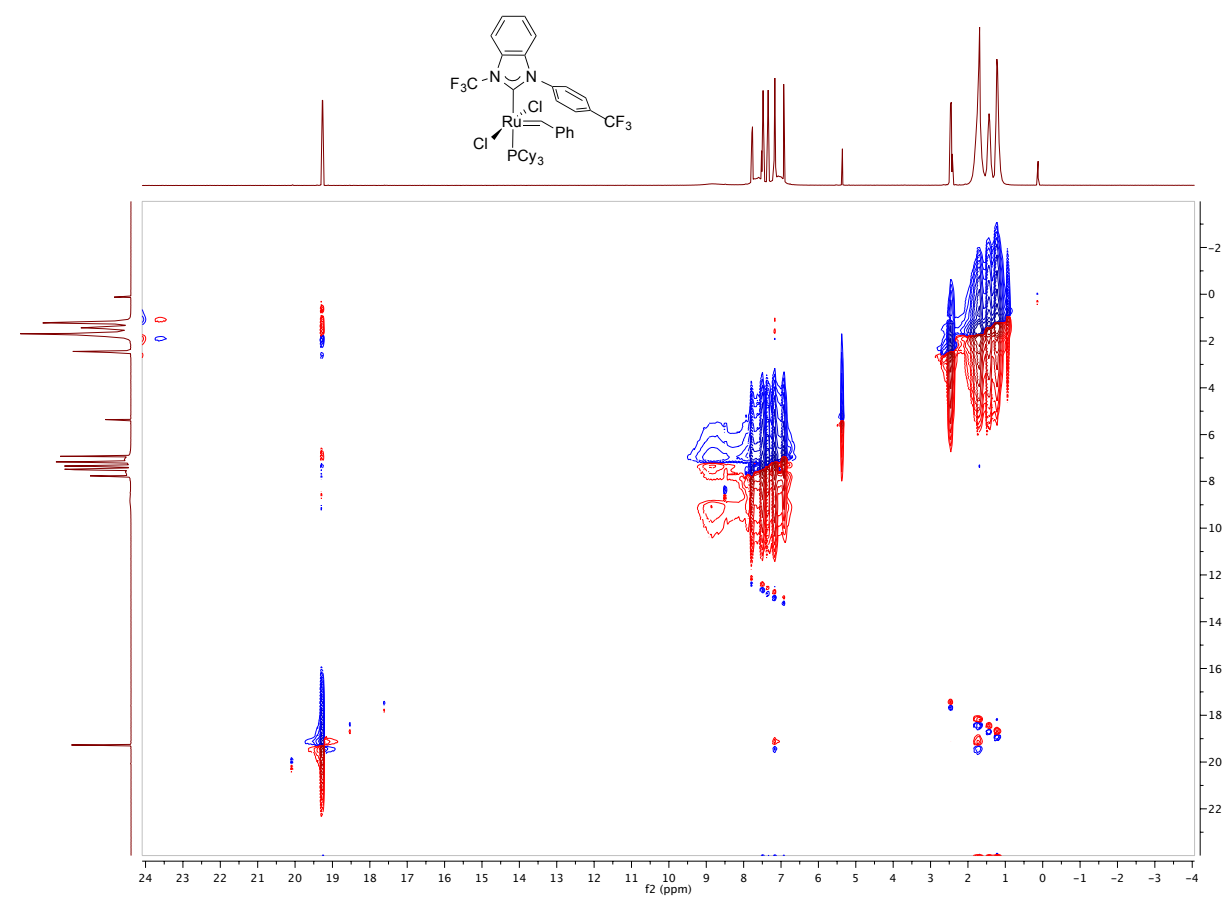

Figure S21. 2D ${ }^{1} \mathrm{H} /{ }^{1} \mathrm{H}$ NOESY NMR spectrum of $6 \mathbf{b}$. Cross peaks between the benzylidene proton and the $\mathrm{N}$-aryl ortho-hydrogens. 


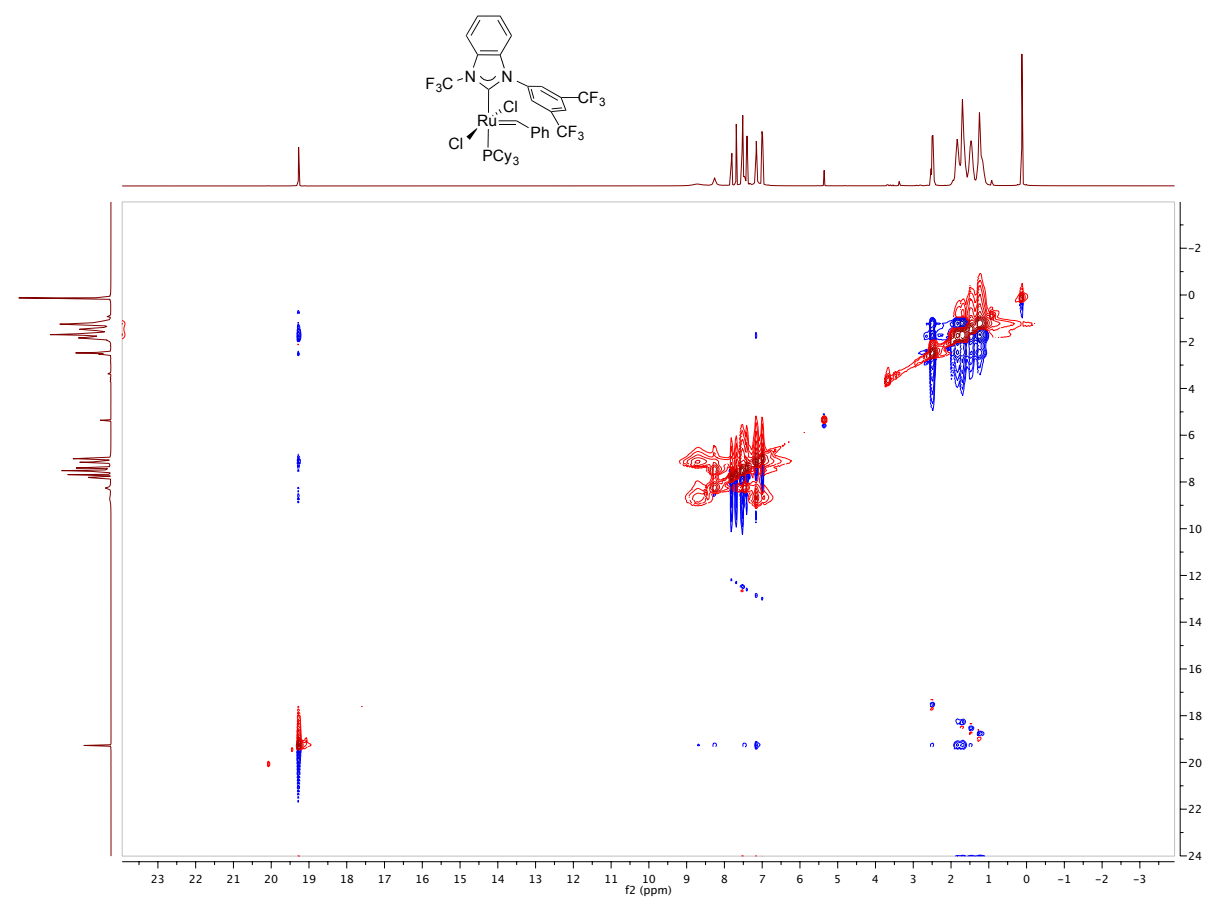

Figure S22. 2D ${ }^{1} \mathrm{H} /{ }^{1} \mathrm{H}$ NOESY NMR spectrum of 6c. Cross peaks between the benzylidene proton and the $\mathrm{N}$-aryl ortho-hydrogens.

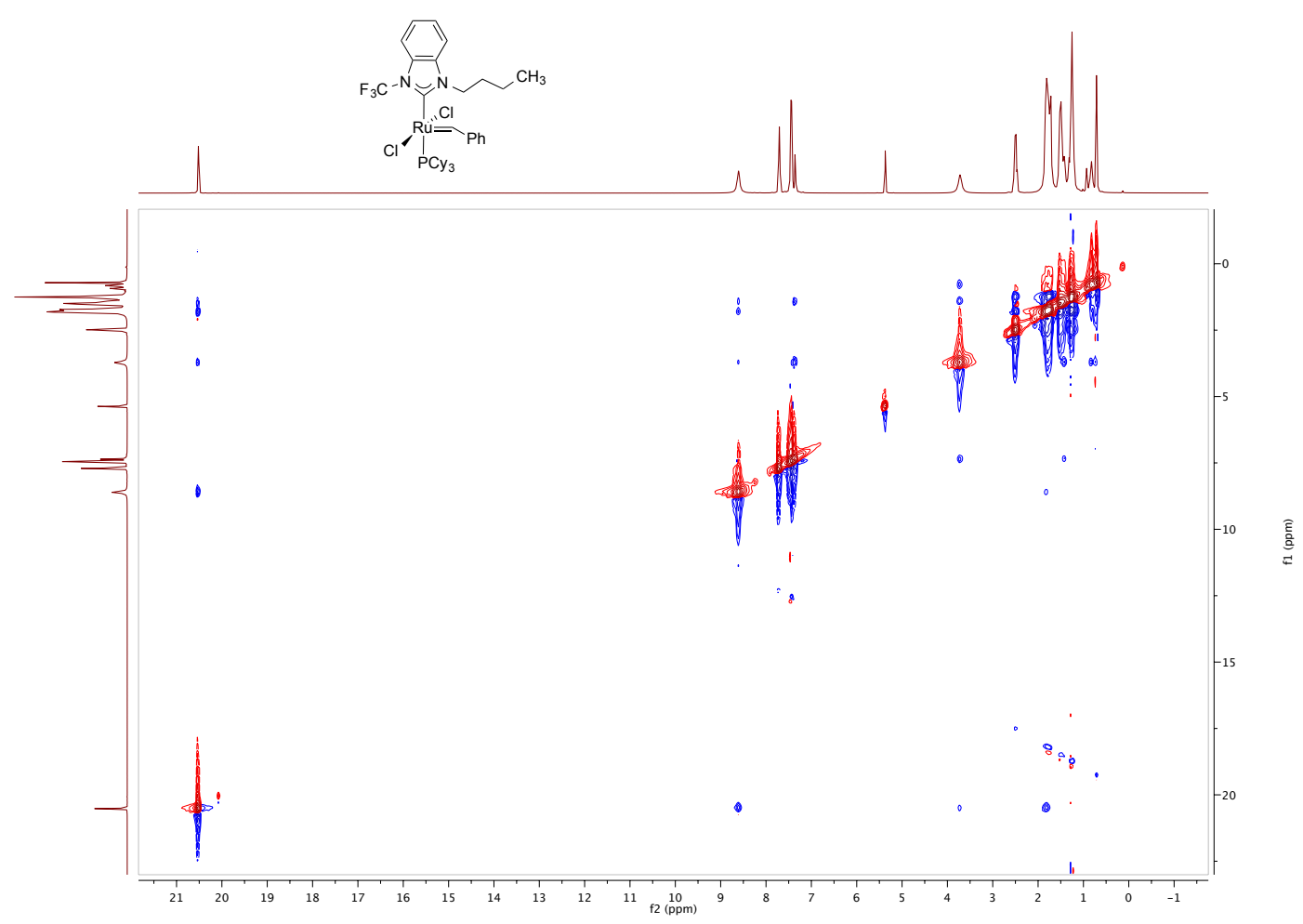

Figure S23. 2D ${ }^{1} \mathrm{H} /{ }^{1} \mathrm{H}$ NOESY NMR spectrum of 6d. Cross peaks between the benzylidene proton and the $\mathrm{N}-n$-butyl methylene hydrogens. 


\section{Crystallographic Analysis}

Table S3. Crystal and Data Collection parameters of complexes $\mathbf{6 a}-\mathbf{b}$ and $\mathbf{6 d}$

\begin{tabular}{|c|c|c|c|}
\hline Identification code & $6 a$ & $6 \mathbf{b}$ & 6d \\
\hline Empirical formula & $\mathrm{C}_{40} \mathrm{H}_{50} \mathrm{Cl}_{4} \mathrm{~F}_{3} \mathrm{~N}_{2} \mathrm{PRu}$ & $\mathrm{C}_{41} \mathrm{H}_{49} \mathrm{Cl}_{4} \mathrm{~F}_{6} \mathrm{~N}_{2} \mathrm{PRu}$ & $\mathrm{C}_{38} \mathrm{H}_{53} \mathrm{Cl}_{4} \mathrm{~F}_{3} \mathrm{~N}_{2} \mathrm{PRu}$ \\
\hline Formula weight & 889.66 & 957.66 & 868.66 \\
\hline Temperature/K & 101.8 & 103.1 & 100 \\
\hline Crystal system & monoclinic & triclinic & triclinic \\
\hline Space group & $\mathrm{P} 2{ }_{1} / \mathrm{c}$ & P-1 & $\mathrm{P}-1$ \\
\hline $\mathrm{a} / \AA$ & $9.6135(17)$ & $10.3182(6)$ & $14.4269(11)$ \\
\hline $\mathrm{b} / \AA$ & $9.9787(17)$ & $13.8690(7)$ & $16.0783(12)$ \\
\hline $\mathrm{c} / \AA$ & $43.076(7)$ & $15.4984(9)$ & $19.3107(14)$ \\
\hline$\alpha /^{\circ}$ & 90.00 & $86.204(2)$ & $105.2320(10)$ \\
\hline$\beta /{ }^{\circ}$ & $90.906(2)$ & $75.373(2)$ & $108.4330(10)$ \\
\hline$\gamma /{ }^{\circ}$ & 90.00 & $75.216(2)$ & $98.5070(10)$ \\
\hline Volume $/ \AA^{3}$ & $4131.8(12)$ & $2074.9(2)$ & $3967.5(5)$ \\
\hline Z & 4 & 2 & 4 \\
\hline$\rho_{\text {calc }} \mathrm{g} / \mathrm{cm}^{3}$ & 1.430 & 1.533 & 1.454 \\
\hline$\mu / \mathrm{mm}^{-1}$ & 0.721 & 0.734 & 0.749 \\
\hline $\mathrm{F}(000)$ & 1832.0 & 980.0 & 1796.0 \\
\hline Crystal size $/ \mathrm{mm}^{3}$ & $0.325 \times 0.322 \times 0.149$ & $0.506 \times 0.446 \times 0.259$ & $0.372 \times 0.325 \times 0.154$ \\
\hline Radiation & $\operatorname{MoK} \alpha(\lambda=0.71073)$ & $\operatorname{MoK} \alpha(\lambda=0.71073)$ & $\operatorname{MoK} \alpha(\lambda=0.71073)$ \\
\hline 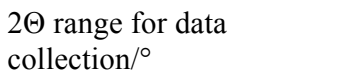 & 4.614 to 60.06 & 4.542 to 61.016 & 2.354 to 56.564 \\
\hline Index ranges & $\begin{array}{l}-13 \leq \mathrm{h} \leq 13,-14 \leq \mathrm{k} \leq 14, \\
-58 \leq 1 \leq 60\end{array}$ & $\begin{array}{l}-14 \leq \mathrm{h} \leq 14,-19 \leq \mathrm{k} \leq 19 \\
-22 \leq 1 \leq 22\end{array}$ & $\begin{array}{l}-19 \leq \mathrm{h} \leq 19,-21 \leq \mathrm{k} \leq 21, \\
-25 \leq 1 \leq 25\end{array}$ \\
\hline Reflections collected & 61512 & 107909 & 41906 \\
\hline Independent reflections & $\begin{array}{l}12056\left[\mathrm{R}_{\text {int }}=0.1048,\right. \\
\left.\mathrm{R}_{\text {sigma }}=0.0 .0908\right]\end{array}$ & $\begin{array}{l}12662\left[\mathrm{R}_{\text {int }}=0.0722,\right. \\
\left.\mathrm{R}_{\text {sigma }}=0.0 .0470\right]\end{array}$ & $\begin{array}{l}19564\left[\mathrm{R}_{\text {int }}=0.0231,\right. \\
\left.\mathrm{R}_{\text {sigma }}=0.0 .0341\right]\end{array}$ \\
\hline Data/restraints/parameters & $12056 / 102 / 491$ & $12662 / 72 / 665$ & $19564 / 0 / 885$ \\
\hline Goodness-of-fit on $\mathrm{F}^{2}$ & 1.076 & 1.057 & 1.047 \\
\hline $\begin{array}{l}\text { Final } \mathrm{R} \text { indexes }[\mathrm{I}>=2 \sigma \\
\text { (I)] }\end{array}$ & $\begin{array}{l}\mathrm{R}_{1}=0.0675 \\
\mathrm{wR}_{2}=0.1063\end{array}$ & $\begin{array}{l}\mathrm{R}_{1}=0.0436 \\
\mathrm{wR}_{2}=0.0654\end{array}$ & $\begin{array}{l}\mathrm{R}_{1}=0.0360 \\
\mathrm{wR}_{2}=0.0934\end{array}$ \\
\hline Final $\mathrm{R}$ indexes [all data] & $\begin{array}{l}\mathrm{R}_{1}=0.1109 \\
\mathrm{wR}_{2}=0.1185\end{array}$ & $\begin{array}{l}\mathrm{R}_{1}=0.0654 \\
\mathrm{wR}_{2}=0.0941\end{array}$ & $\begin{array}{l}\mathrm{R}_{1}=0.0430 \\
\mathrm{wR}_{2}=0.0984\end{array}$ \\
\hline $\begin{array}{l}\text { Largest diff. peak/hole / e } \\
\AA^{-3}\end{array}$ & $0.93 /-1.08$ & $1.10 /-1.30$ & $1.68 /-1.42$ \\
\hline
\end{tabular}




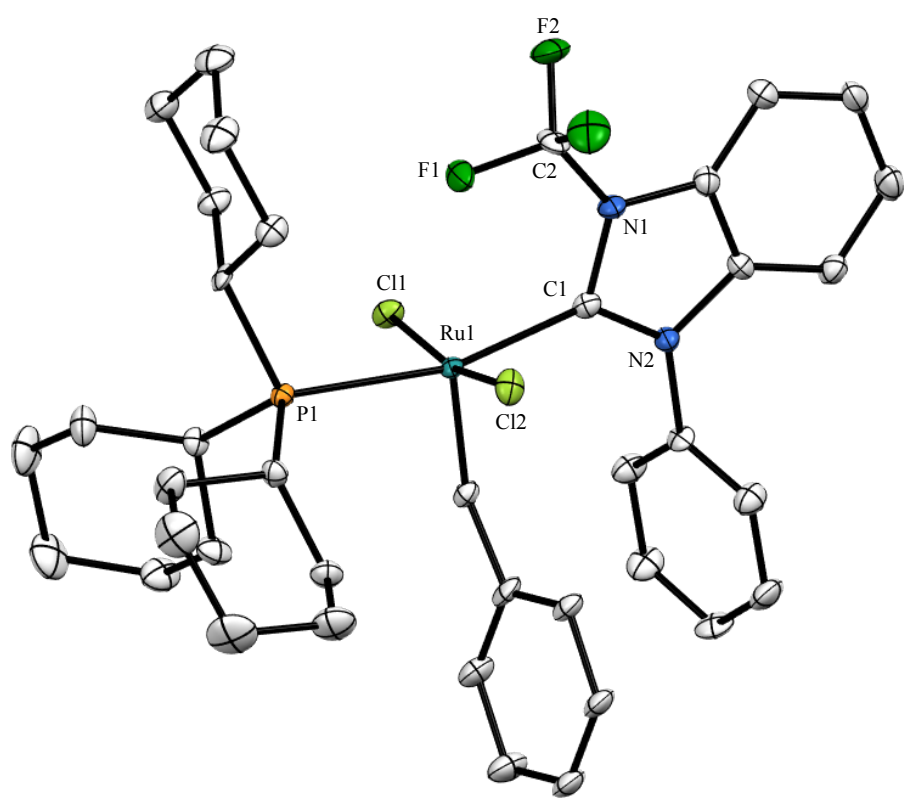

Figure S24. Ortep drawing of 6a. Hydrogen atoms are omitted for clarity, and thermal ellipsoids are set to $50 \%$ probability. Selected bond lengths $[\AA]$, bond angles [deg], and torsion angles [deg]: Ru1-F1 2.652(2), C2-F1 1.335(4), C2-F2 1.324(5), N1-C1-Ru1 123.1(2), N2-C1-Ru1 133.5(3), Cl1-Ru1-C12 168.28(2), C1-N1-C2-F1 $18.9(5)$.

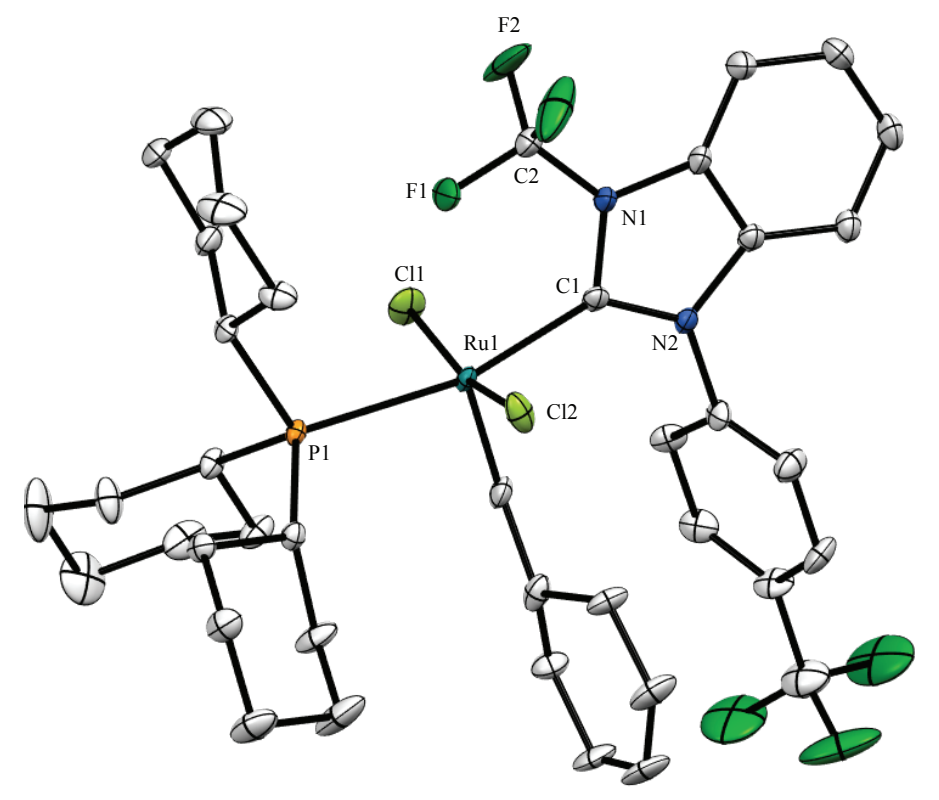

Figure S25. Ortep drawing of $\mathbf{6 b}$. Hydrogen atoms are omitted for clarity, and thermal ellipsoids are set to $50 \%$ probability. Selected bond lengths $[\AA]$, bond angles [deg], and torsion angles [deg]: Ru1-F1 2.630(2), C2-F1 1.311(3), C2-F2 1.304(4), N1-C1-Ru1 123.1(2), N2-C1-Ru1 133.5(2), Cl1-Ru1-C12 166.33(2), C1-N1-C2-F1 $1.6(3)$. 


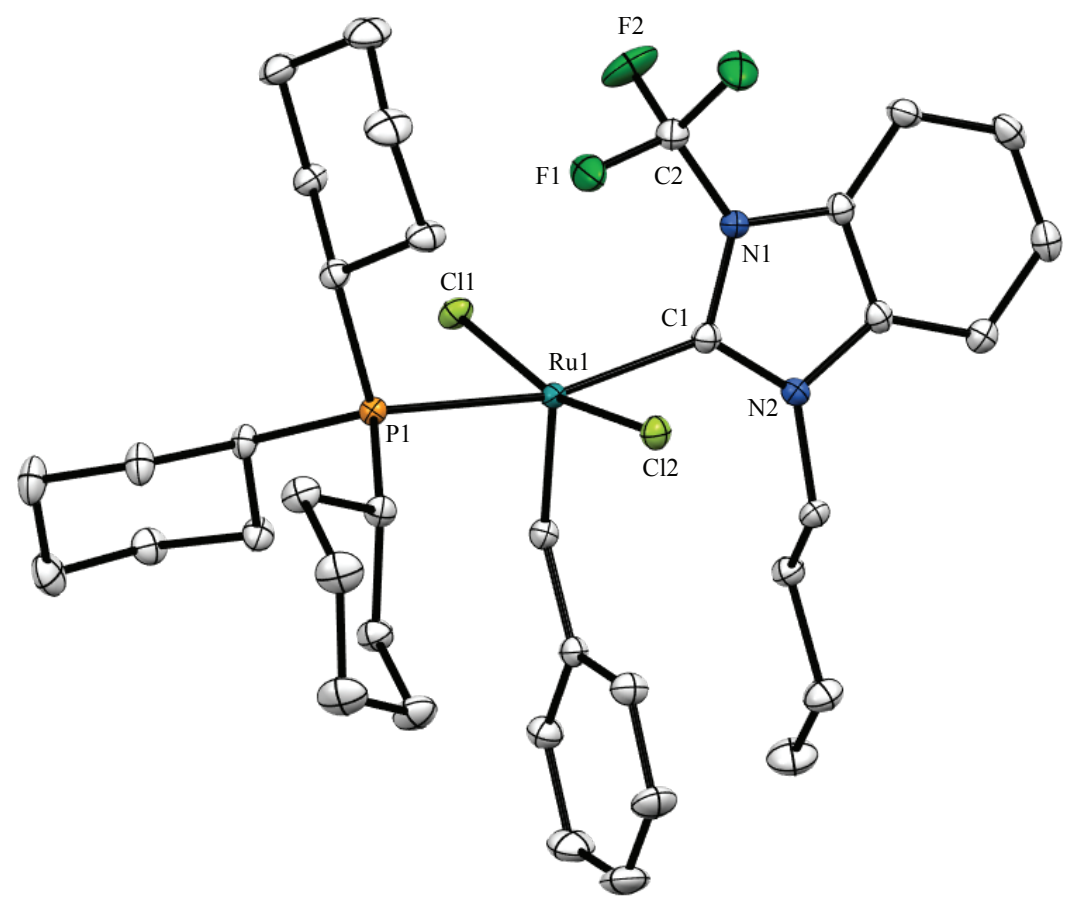

Figure S26. Ortep drawing of 6d. Hydrogen atoms are omitted for clarity, and thermal ellipsoids are set to $50 \%$ probability. Selected bond lengths $[\AA \AA]$, bond angles [deg], and torsion angles [deg]: Ru1-F1 2.629(2), C2-F1 1.332(3), C2-F2 1.328(3), N1-C1-Ru1 121.44(15), N2-C1-Ru1 134.32 (16), C11-Ru1-C12 166.56(2), C1-N1-C2F1 $11.0(3)$. 
Supporting Information for Engl, Fedorov, Copéret, Togni

\section{NMR Spectra}

5.1 Mesityl(4-(trifluoromethyl)phenyl)iodonium tetrafluoroborate (C) ${ }^{1} \mathrm{H}$ NMR (400.20 MHz, $\left.\left(\mathrm{CD}_{3}\right)_{2} \mathrm{SO}\right)$ :

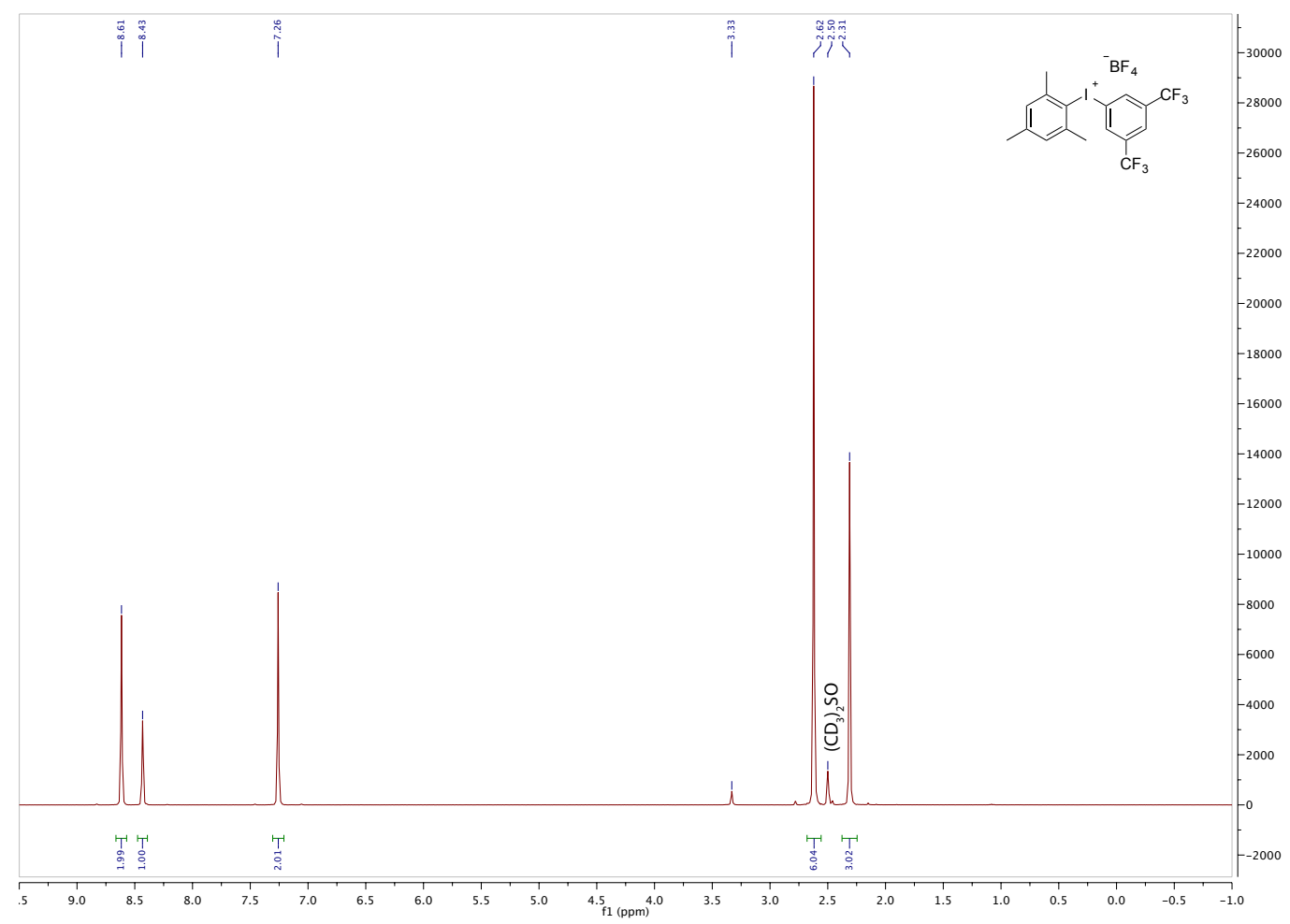

${ }^{19} \mathrm{~F} \mathrm{NMR}\left(376.56 \mathrm{MHz},\left(\mathrm{CD}_{3}\right)_{2} \mathrm{SO}\right)$ :

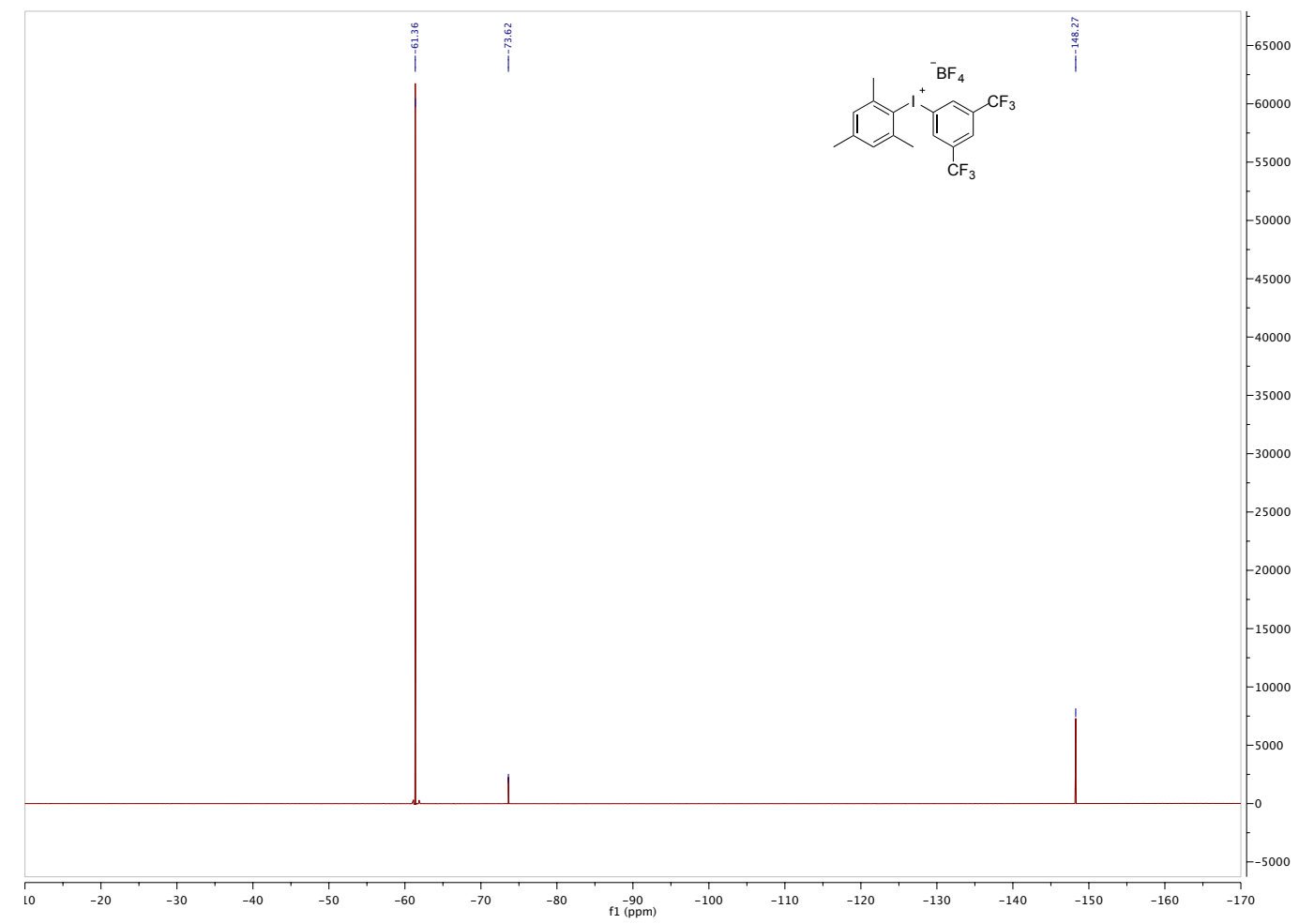


Supporting Information for Engl, Fedorov, Copéret, Togni

${ }^{13} \mathrm{C}\left\{{ }^{1} \mathrm{H}\right\}$ NMR $\left(100.63 \mathrm{MHz},\left(\mathrm{CD}_{3}\right)_{2} \mathrm{SO}\right)$ :

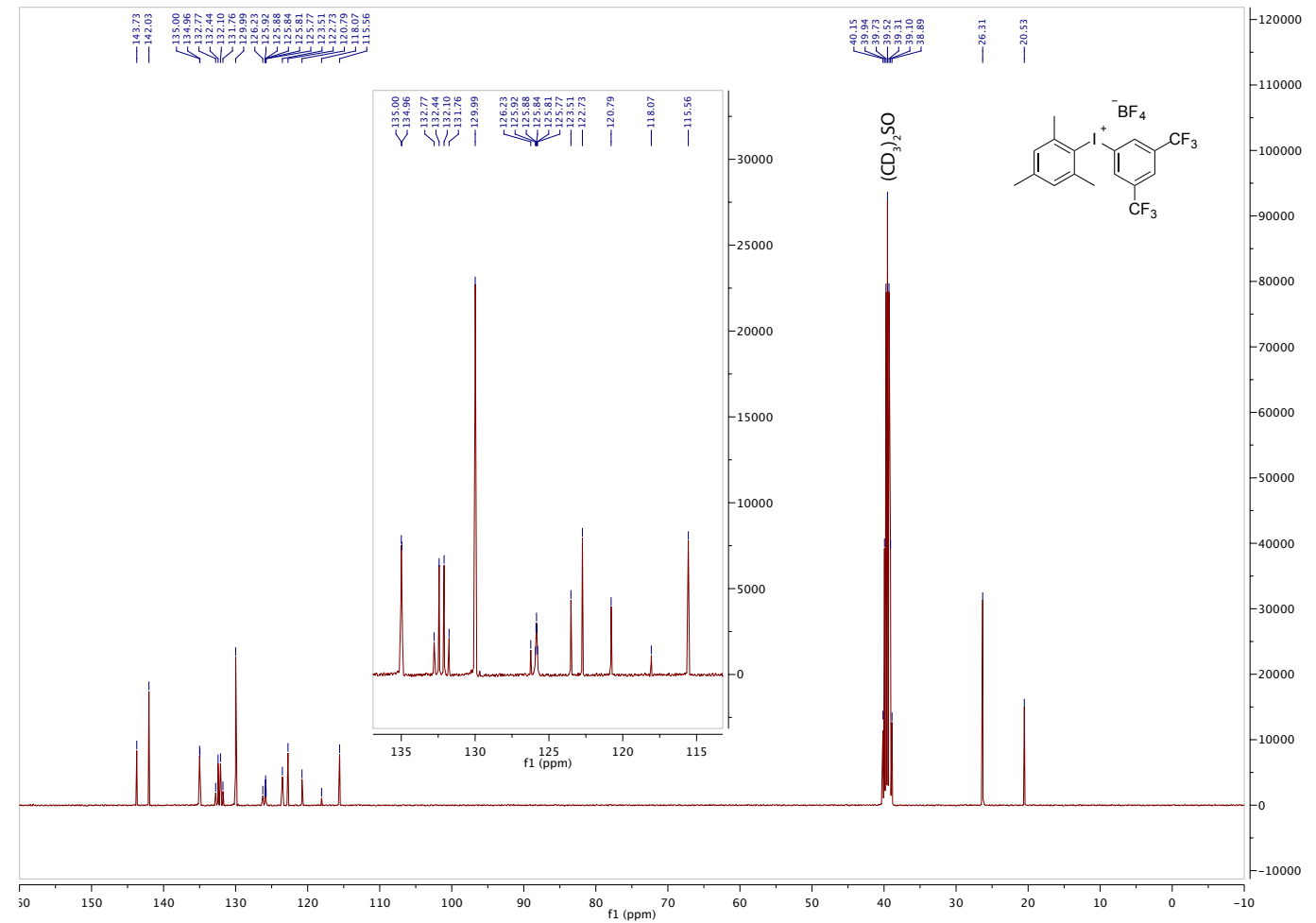

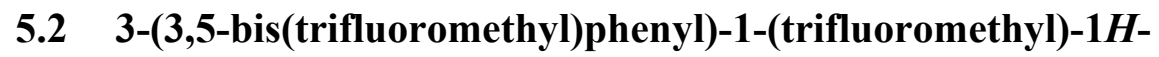
benzo $[d]$ imidazol-3-ium tetrafluoroborate $(5 c)$

${ }^{1} \mathrm{H}$ NMR (300.13 MHz, $\left.\left(\mathrm{CD}_{3}\right)_{2} \mathrm{CO}\right)$ :

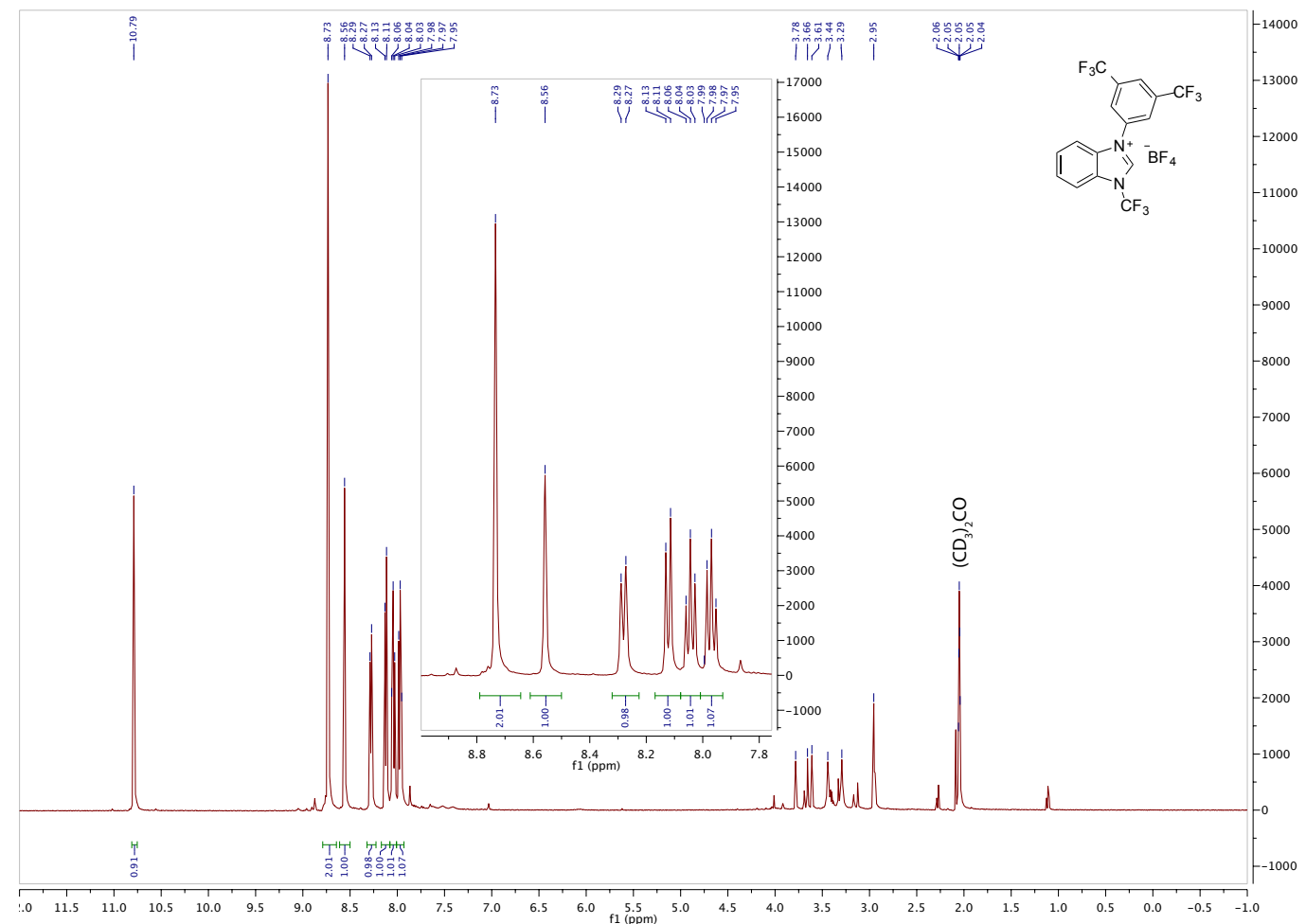


Supporting Information for Engl, Fedorov, Copéret, Togni

${ }^{19}$ F NMR $\left(282.38 \mathrm{MHz},\left(\mathrm{CD}_{3}\right)_{2} \mathrm{CO}\right)$ :

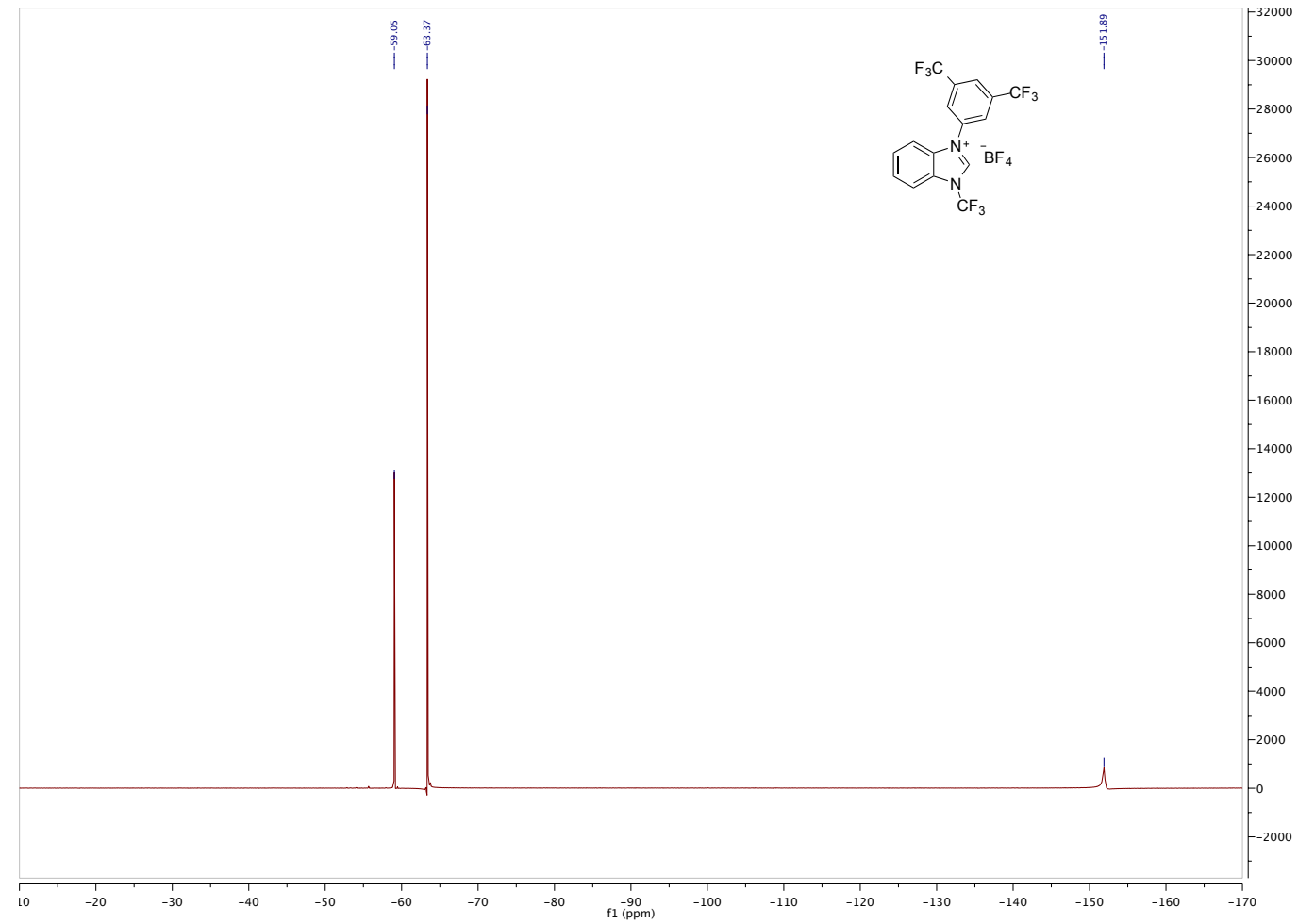

${ }^{13} \mathrm{C}\left\{{ }^{1} \mathrm{H}\right\}$ NMR $\left(75.48 \mathrm{MHz},\left(\mathrm{CD}_{3}\right){ }_{2} \mathrm{CO}\right)$ :

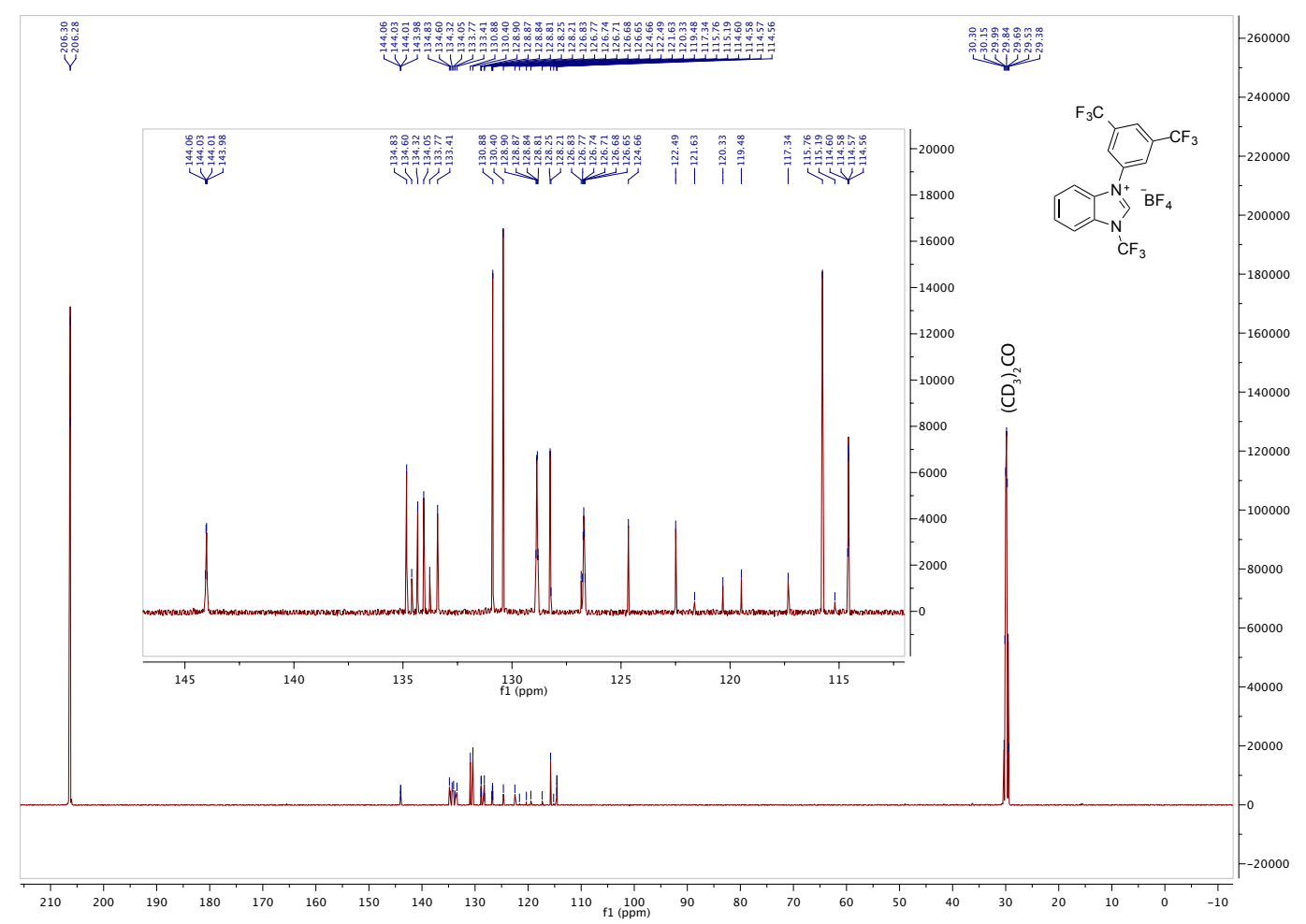


Supporting Information for Engl, Fedorov, Copéret, Togni

\subsection{3-butyl-1-(trifluoromethyl)-1H-benzo[d]imidazol-3-ium} trifluoromethanesulfonate $(5 \mathrm{~d})$

${ }^{1} \mathrm{H}$ NMR (300.13 MHz, $\left.\left(\mathrm{CD}_{3}\right)_{2} \mathrm{SO}\right)$ :

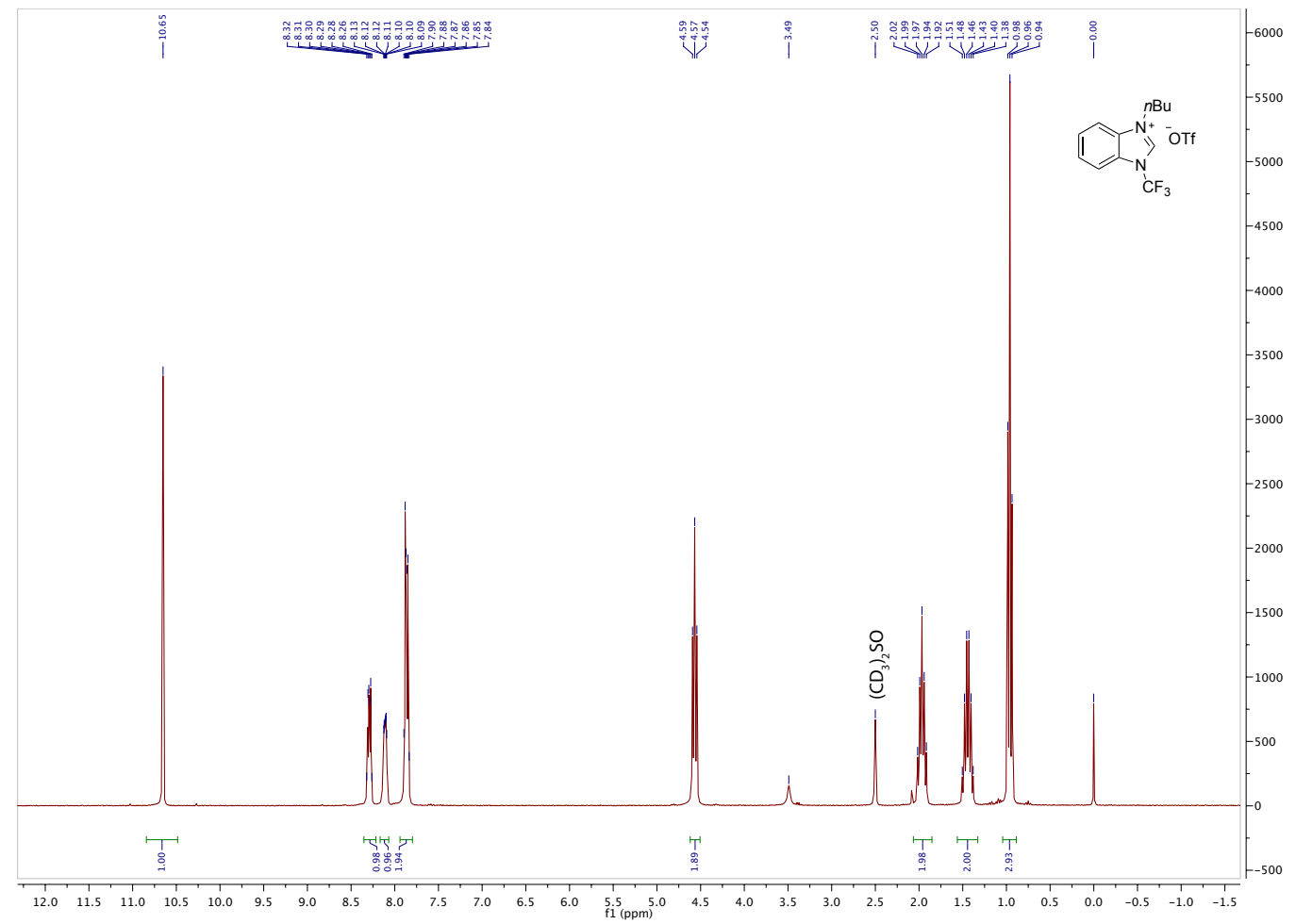

${ }^{19}$ F NMR (282.38 MHz, $\left.\left(\mathrm{CD}_{3}\right)_{2} \mathrm{SO}\right)$ :

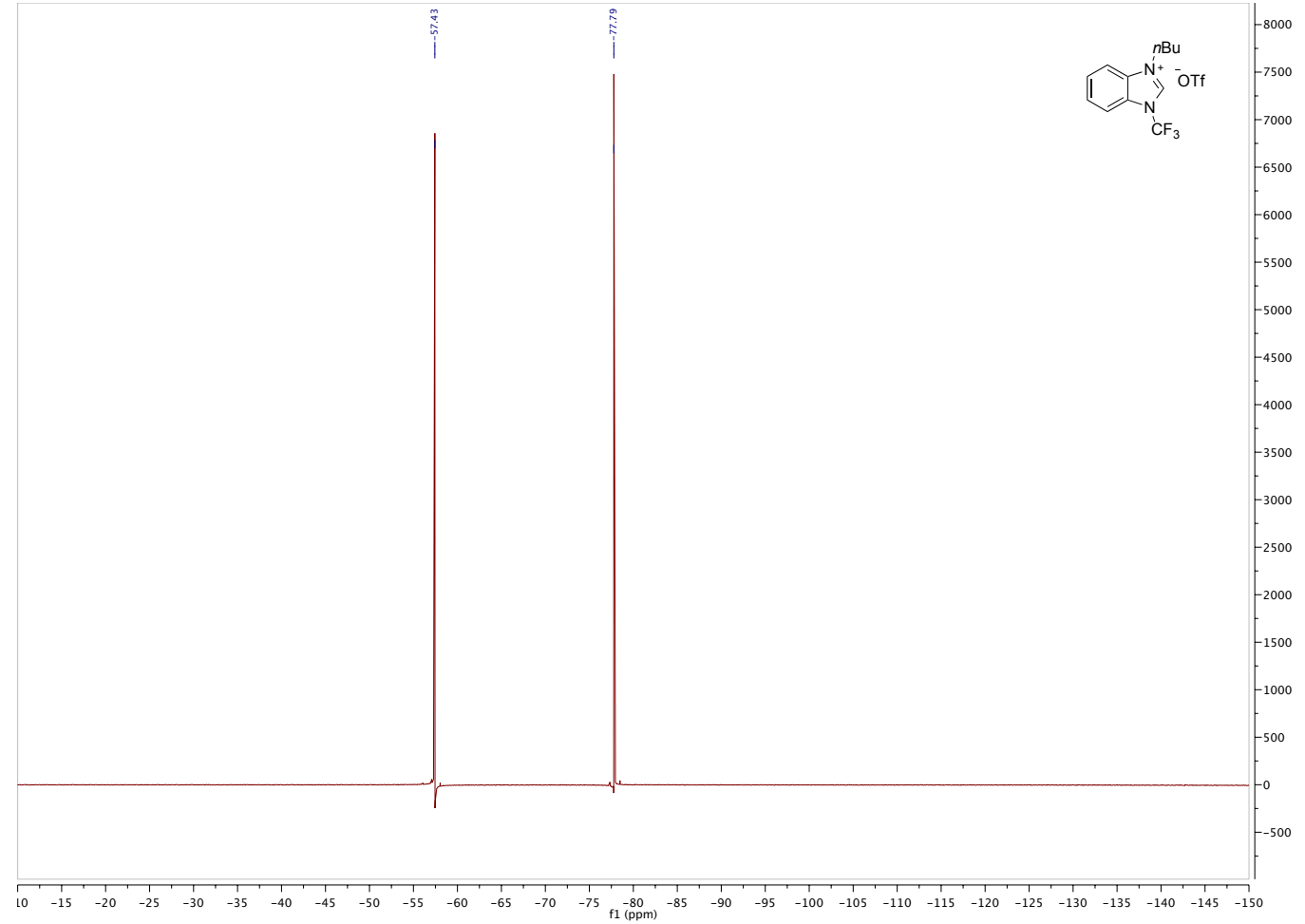


${ }^{13} \mathrm{C}\left\{{ }^{1} \mathrm{H}\right\}$ NMR $\left(75.48 \mathrm{MHz},\left(\mathrm{CD}_{3}\right)_{2} \mathrm{SO}\right)$ :

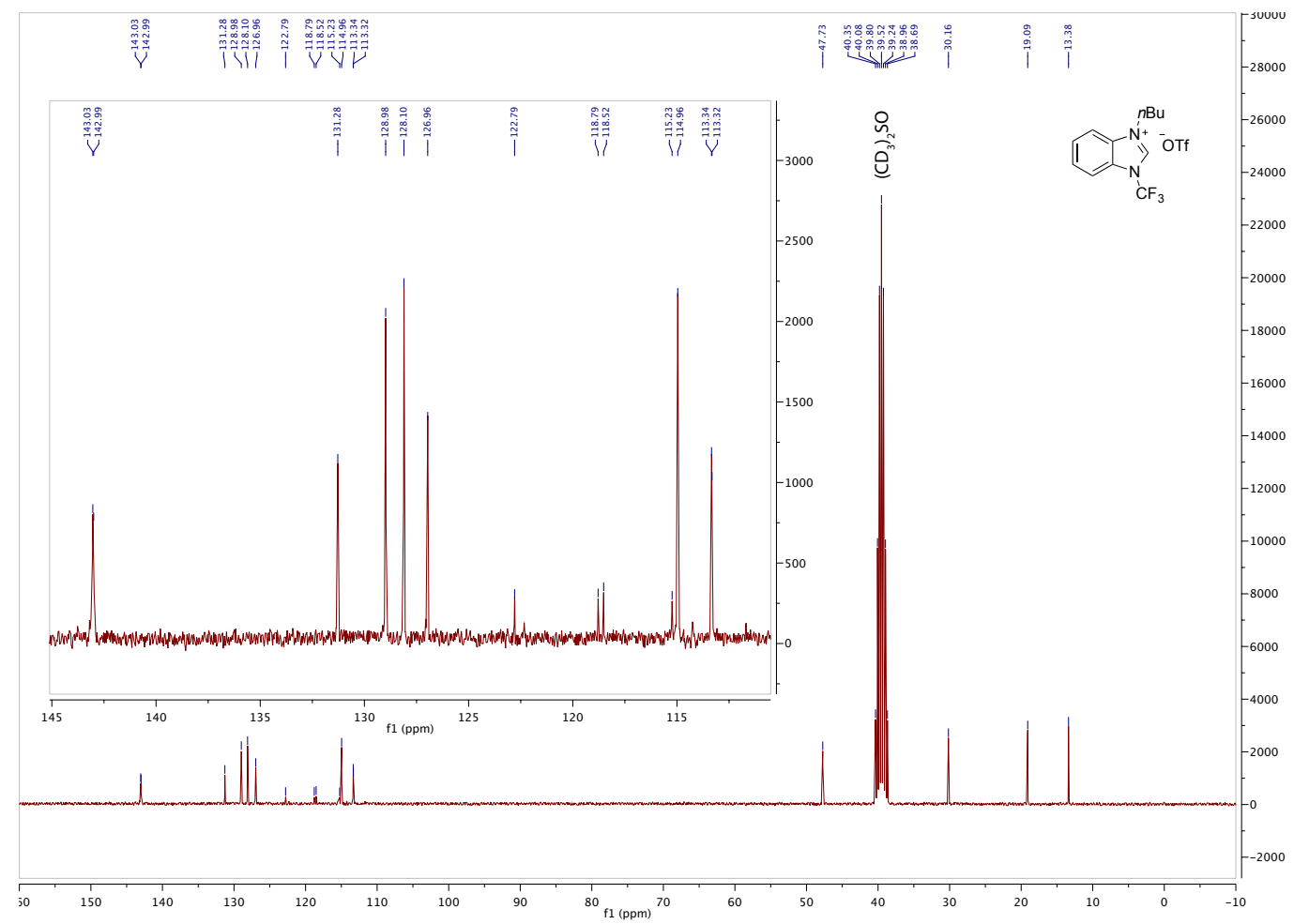

5.4 1-isopropyl-3-(4-(trifluoromethyl)phenyl)-1H-benzo $[d]$ imidazol-3-ium tetrafluoroborate (D)

${ }^{1} \mathrm{H}$ NMR (500.26 MHz, $\left.\left(\mathrm{CD}_{3}\right)_{2} \mathrm{SO}\right)$ :

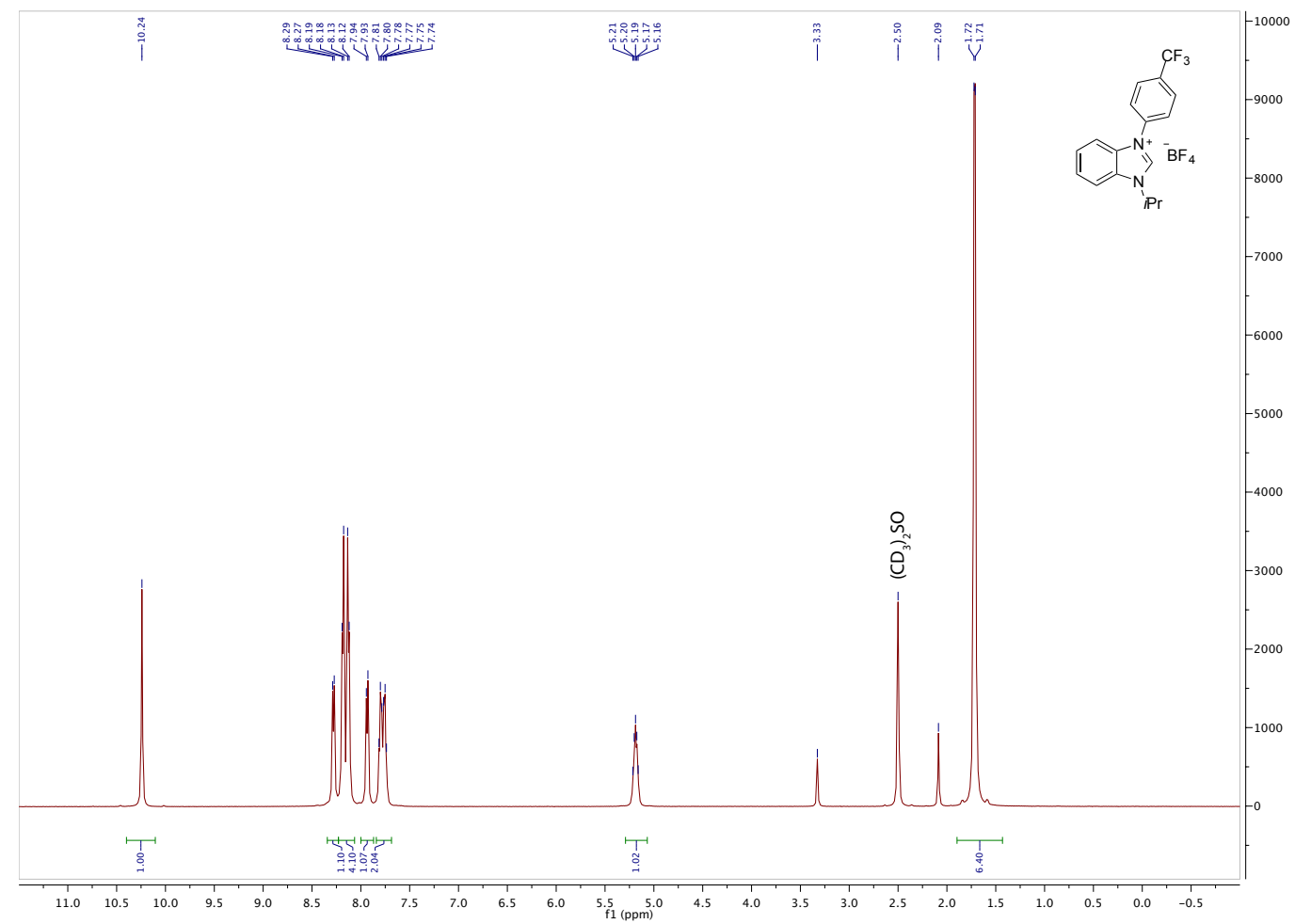


Supporting Information for Engl, Fedorov, Copéret, Togni

${ }^{19}$ F NMR (282.38 MHz, $\left.\left(\mathrm{CD}_{3}\right)_{2} \mathrm{SO}\right)$ :

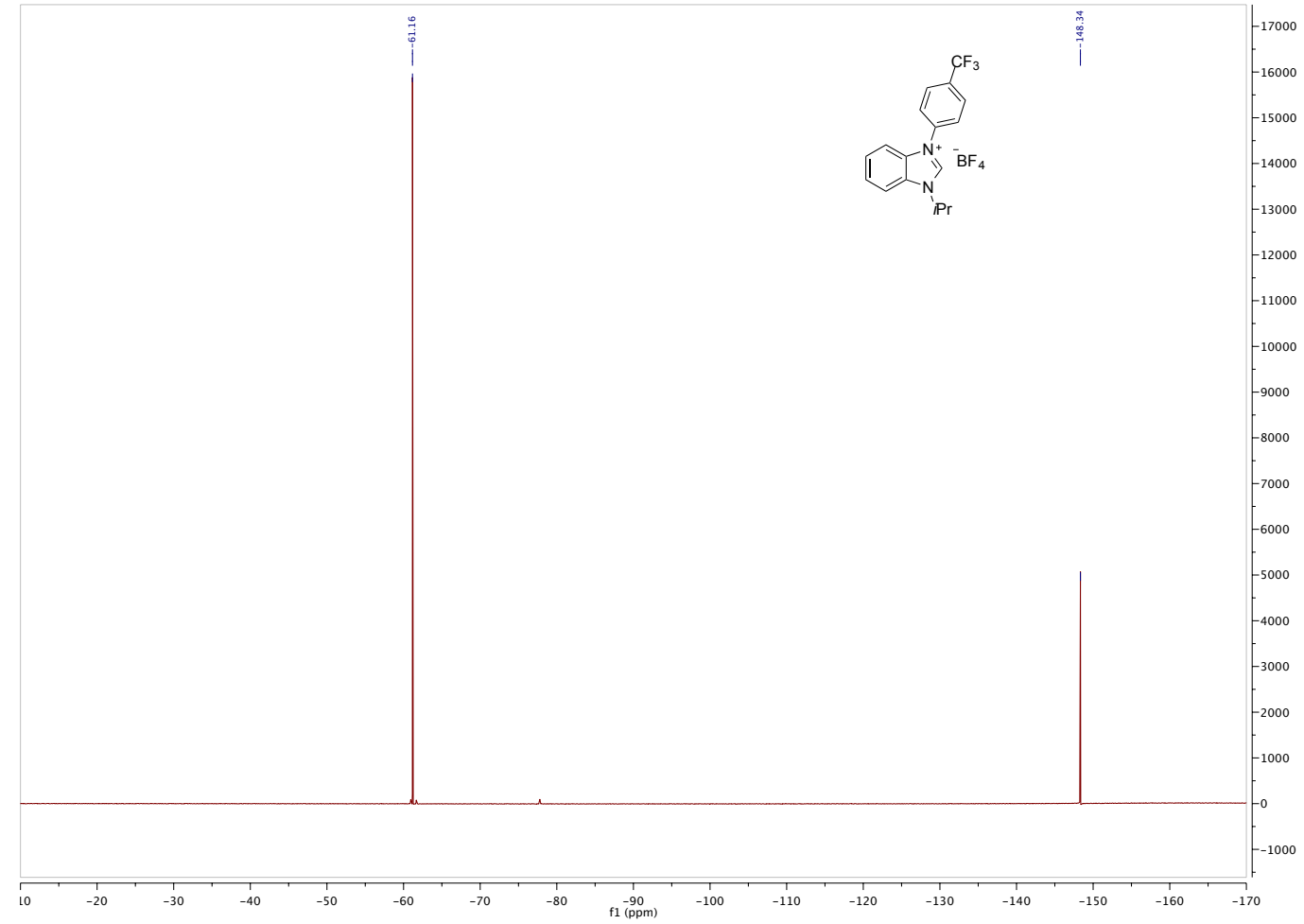

${ }^{13} \mathrm{C}\left\{{ }^{1} \mathrm{H}\right\}$ NMR $\left(125.80 \mathrm{MHz},\left(\mathrm{CD}_{3}\right)_{2} \mathrm{CO}\right)$ :

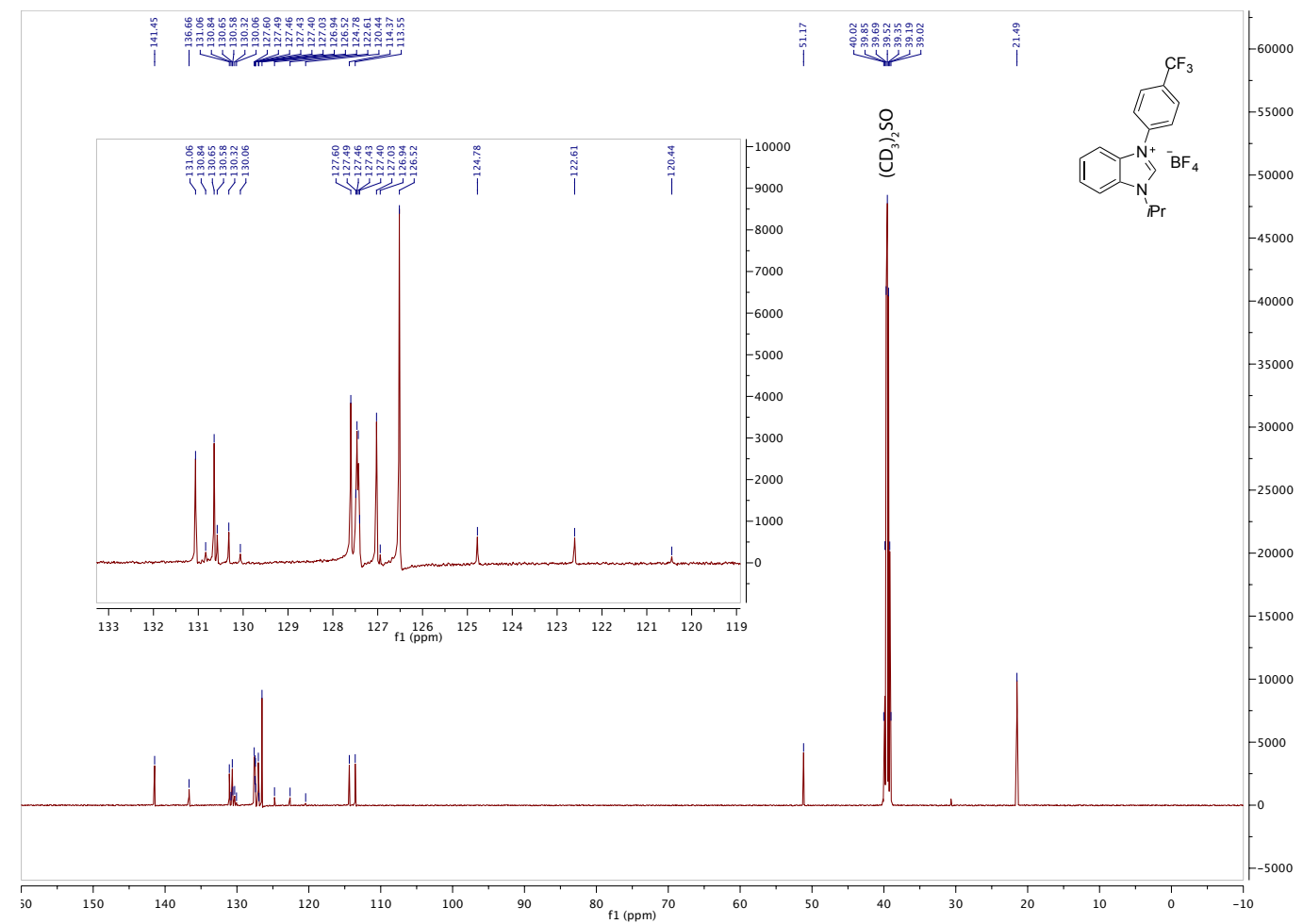


Supporting Information for Engl, Fedorov, Copéret, Togni

5.5 Tricyclohexylphosphine[1-(trifluoromethyl)-3-(phenyl)-1Hbenzo[d]imidazol-3-ylidene][benzylidene]ruthenium dichloride (6a)

${ }^{1} \mathrm{H} \operatorname{NMR}\left(500.27 \mathrm{MHz}, \mathrm{CD}_{2} \mathrm{Cl}_{2}\right)$ :

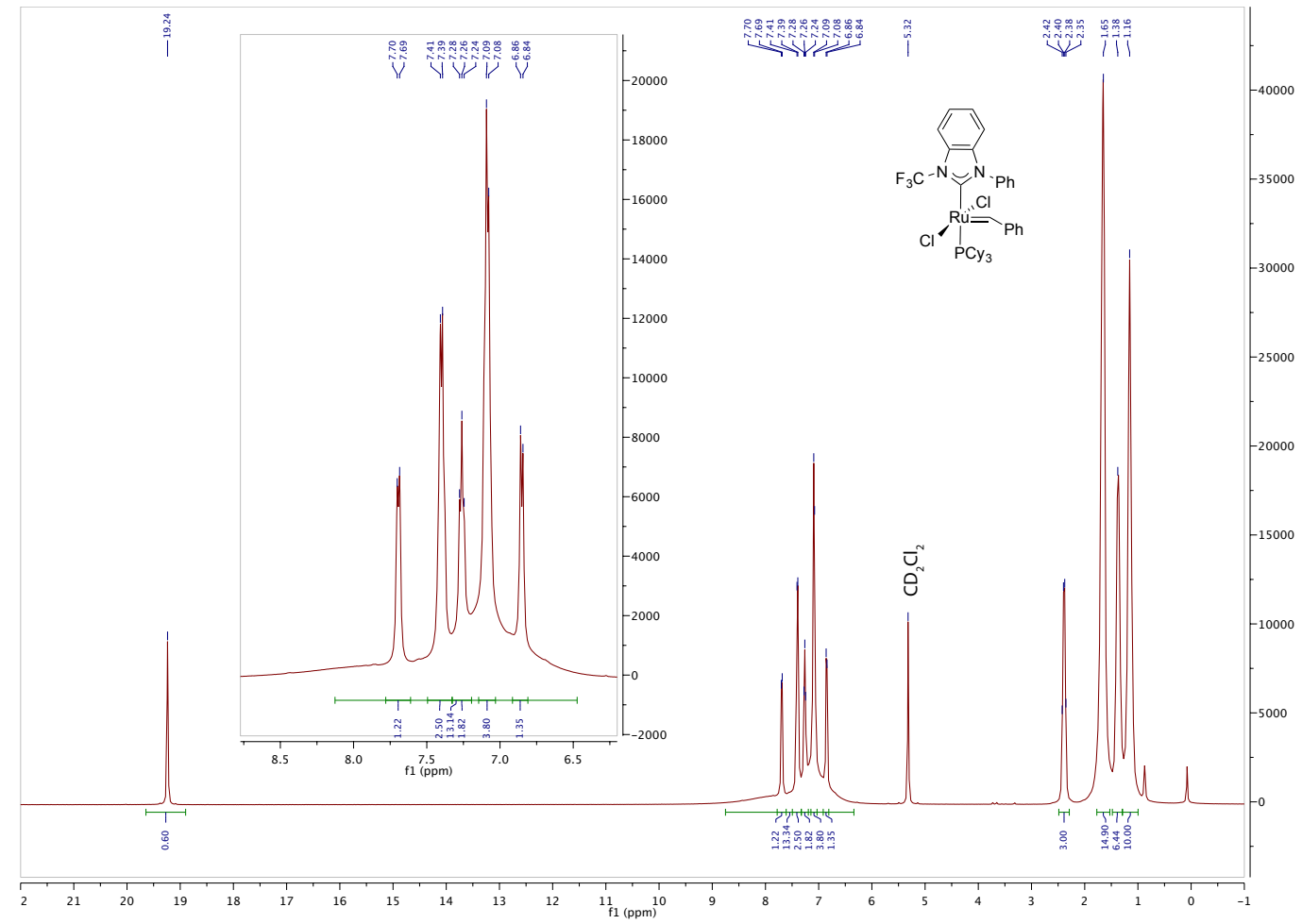

${ }^{19} \mathrm{~F}$ NMR (470.67 MHz, $\left.\mathrm{CD}_{2} \mathrm{Cl}_{2}\right)$ :

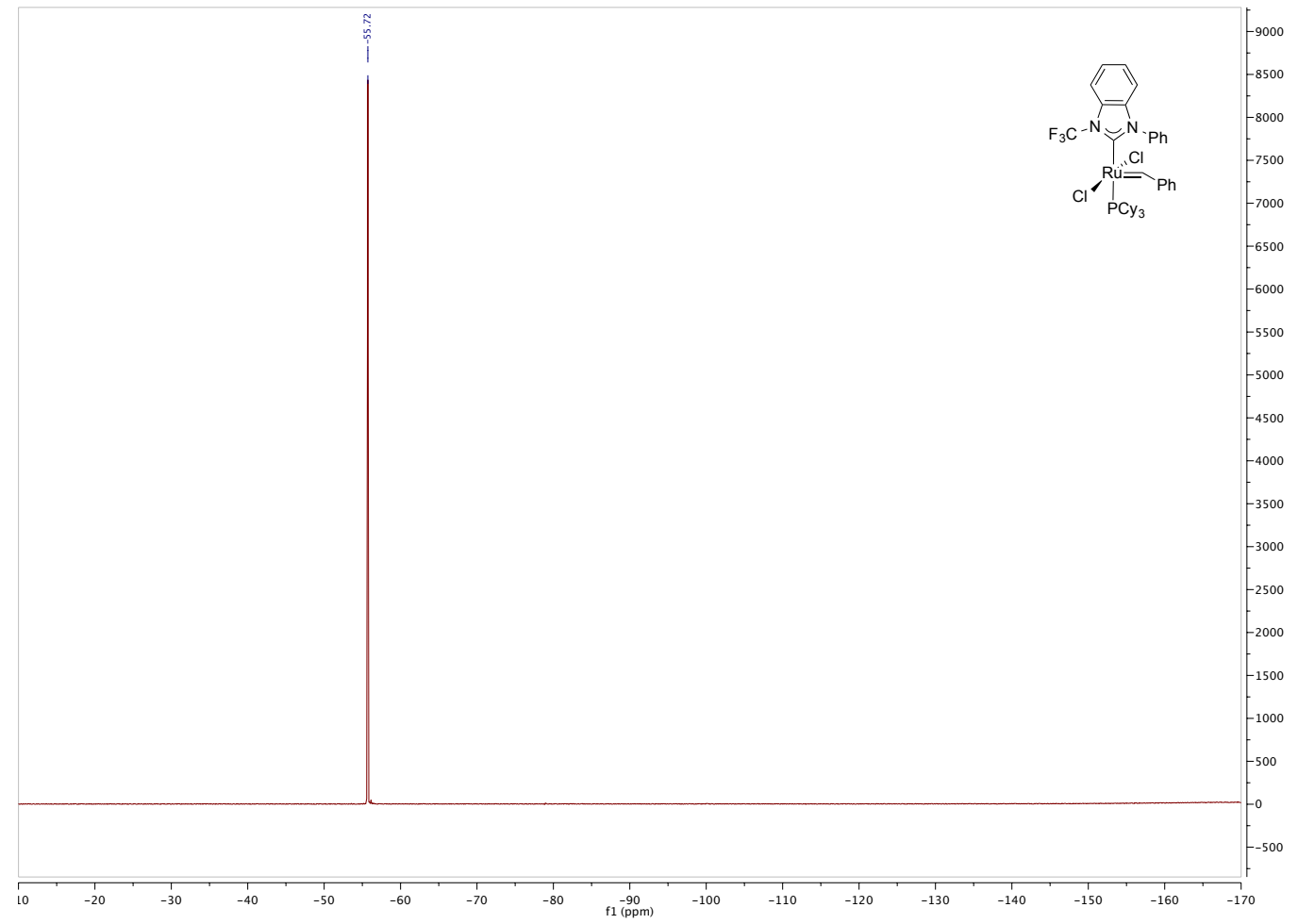


Supporting Information for Engl, Fedorov, Copéret, Togni

${ }^{31} \mathrm{P}$ NMR (202.50 MHz, $\left.\mathrm{CD}_{2} \mathrm{Cl}_{2}\right)$ :

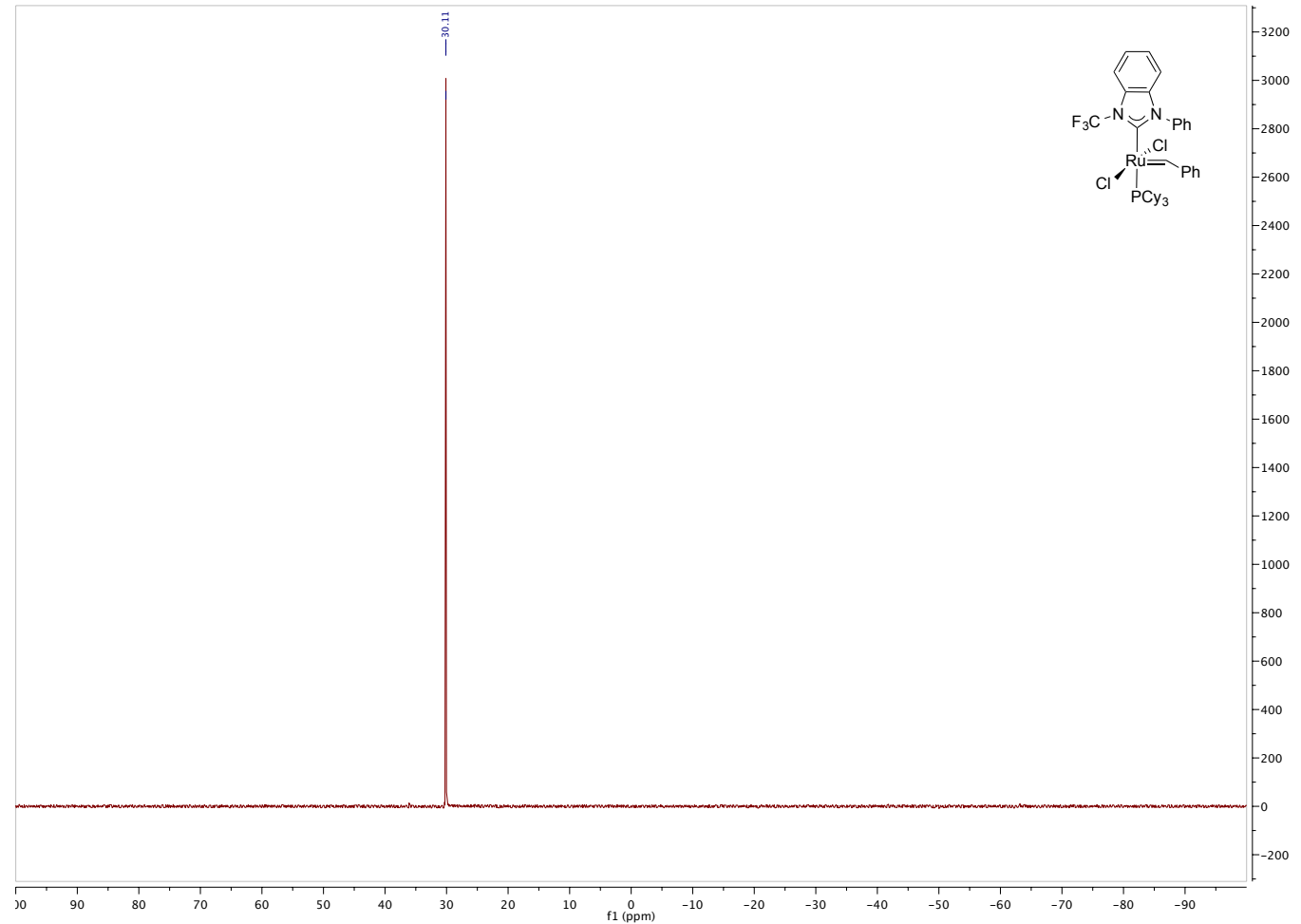

${ }^{13} \mathrm{C}\left\{{ }^{1} \mathrm{H}\right\}$ NMR (125.81 MHz, $\left.\mathrm{CD}_{2} \mathrm{Cl}_{2}\right)$ :

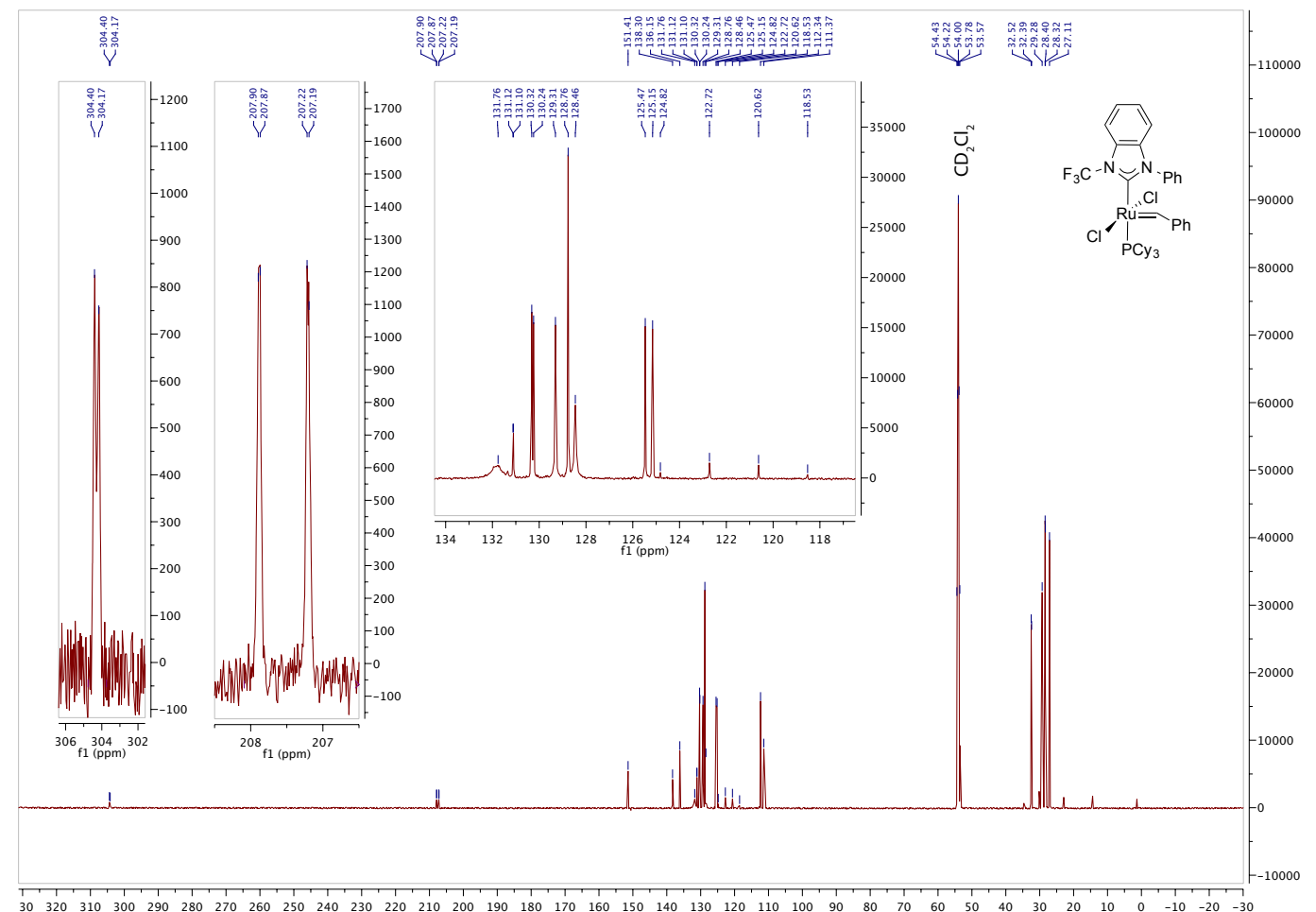


Supporting Information for Engl, Fedorov, Copéret, Togni

5.6 Tricyclohexylphosphine[1-(trifluoromethyl)-3-(4-(trifluoromethyl)phenyl)$1 H$-benzo[d]imidazol-3-ylidene][benzylidene]ruthenium dichloride (6b)

${ }^{1} \mathrm{H} \operatorname{NMR}\left(500.27 \mathrm{MHz}, \mathrm{CD}_{2} \mathrm{Cl}_{2}\right)$ :

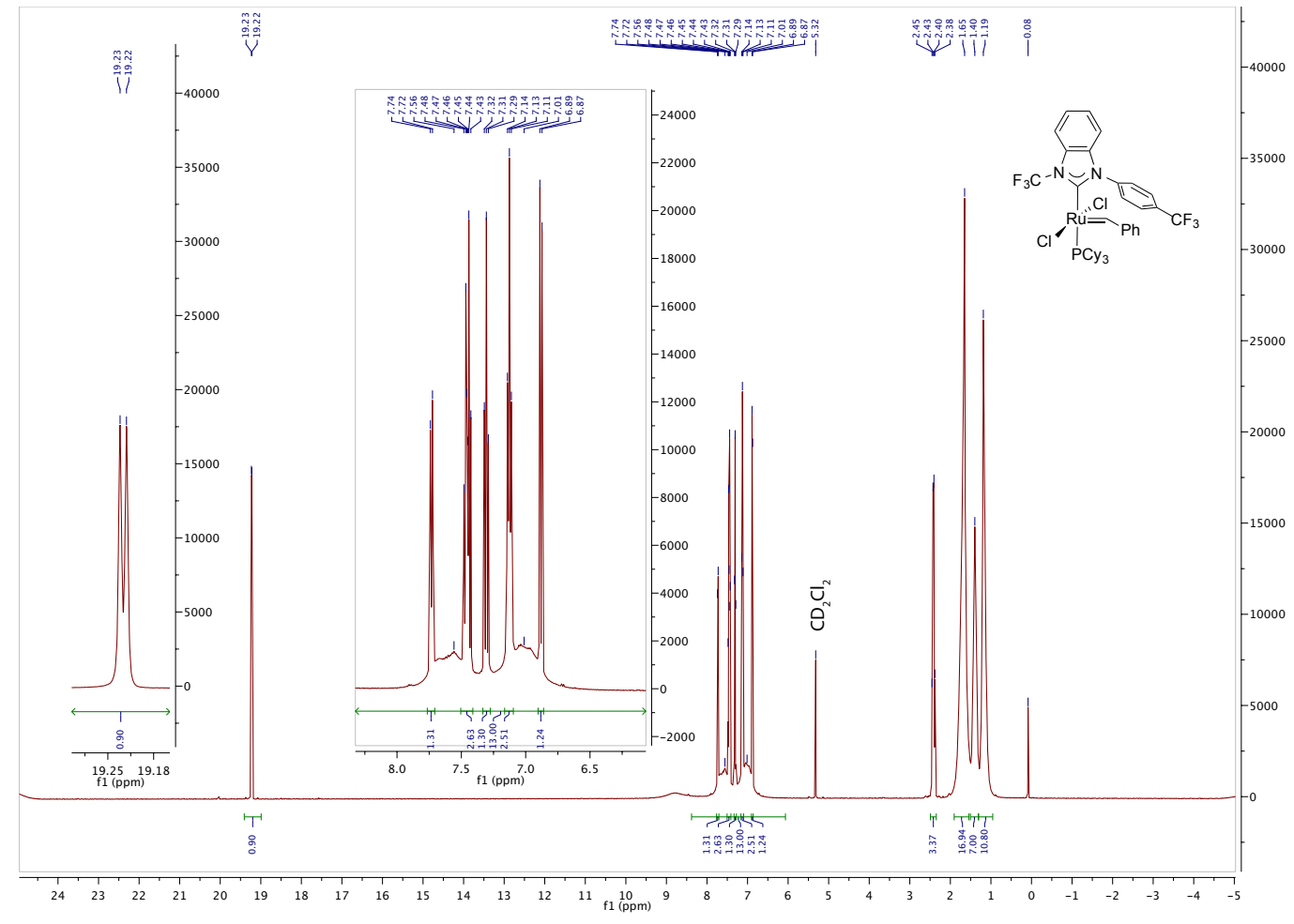

${ }^{19} \mathrm{~F}$ NMR $\left(470.67 \mathrm{MHz}, \mathrm{CD}_{2} \mathrm{Cl}_{2}\right)$ :

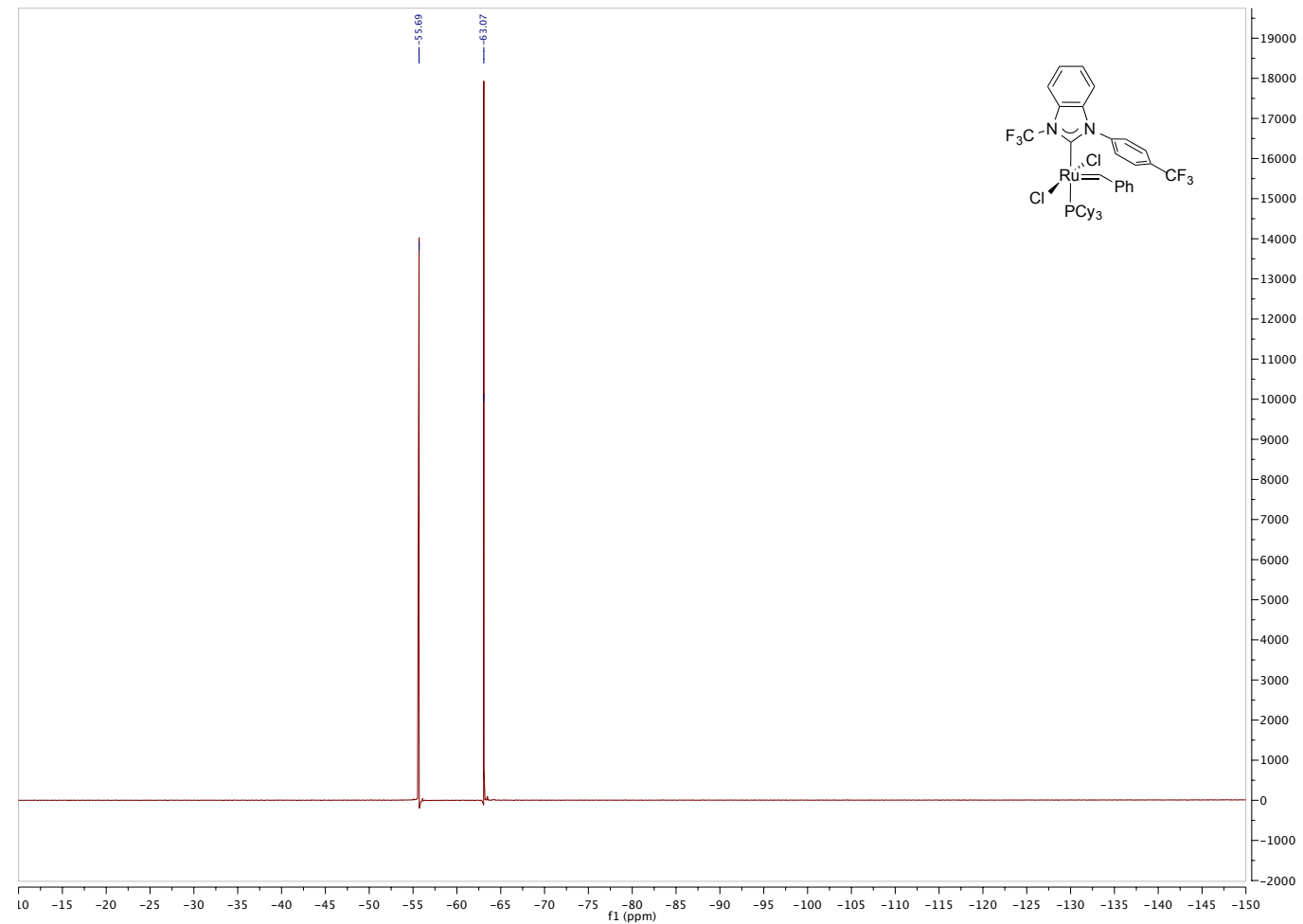


Supporting Information for Engl, Fedorov, Copéret, Togni

${ }^{31} \mathrm{P}$ NMR (202.50 MHz, $\left.\mathrm{CD}_{2} \mathrm{Cl}_{2}\right)$ :

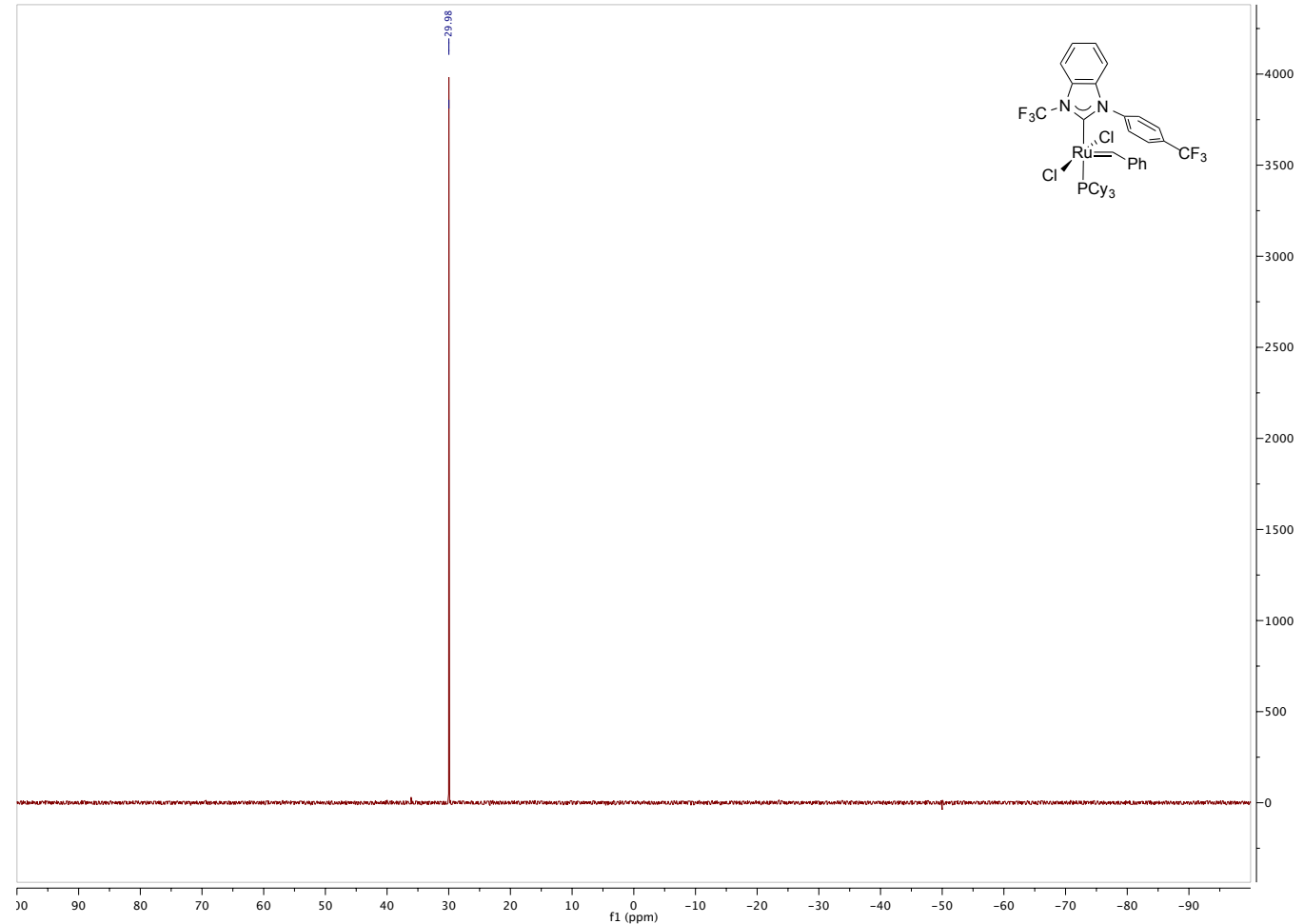

${ }^{13} \mathrm{C}\left\{{ }^{1} \mathrm{H}\right\}$ NMR (125.81 MHz, $\left.\mathrm{CD}_{2} \mathrm{Cl}_{2}\right)$ :

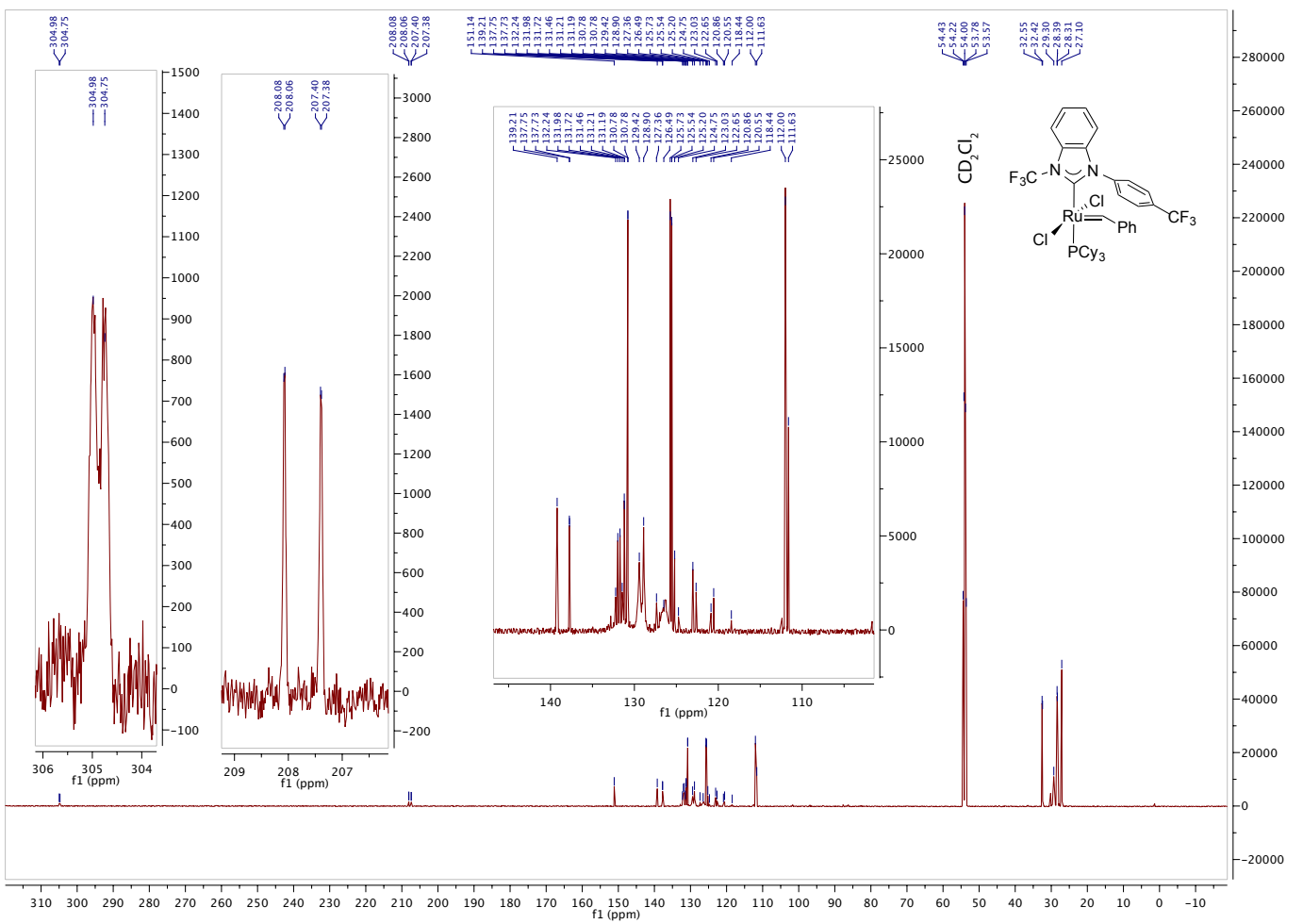


Supporting Information for Engl, Fedorov, Copéret, Togni

5.7 Tricyclohexylphosphine[3-(3,5-bis(trifluoromethyl)phenyl)-1(trifluoromethyl)-1H-benzo[d] imidazol-3-ylidene][benzylidene]ruthenium dichloride (6c)

${ }^{1} \mathrm{H} \mathrm{NMR}\left(500.27 \mathrm{MHz}, \mathrm{CD}_{2} \mathrm{Cl}_{2}\right)$ :

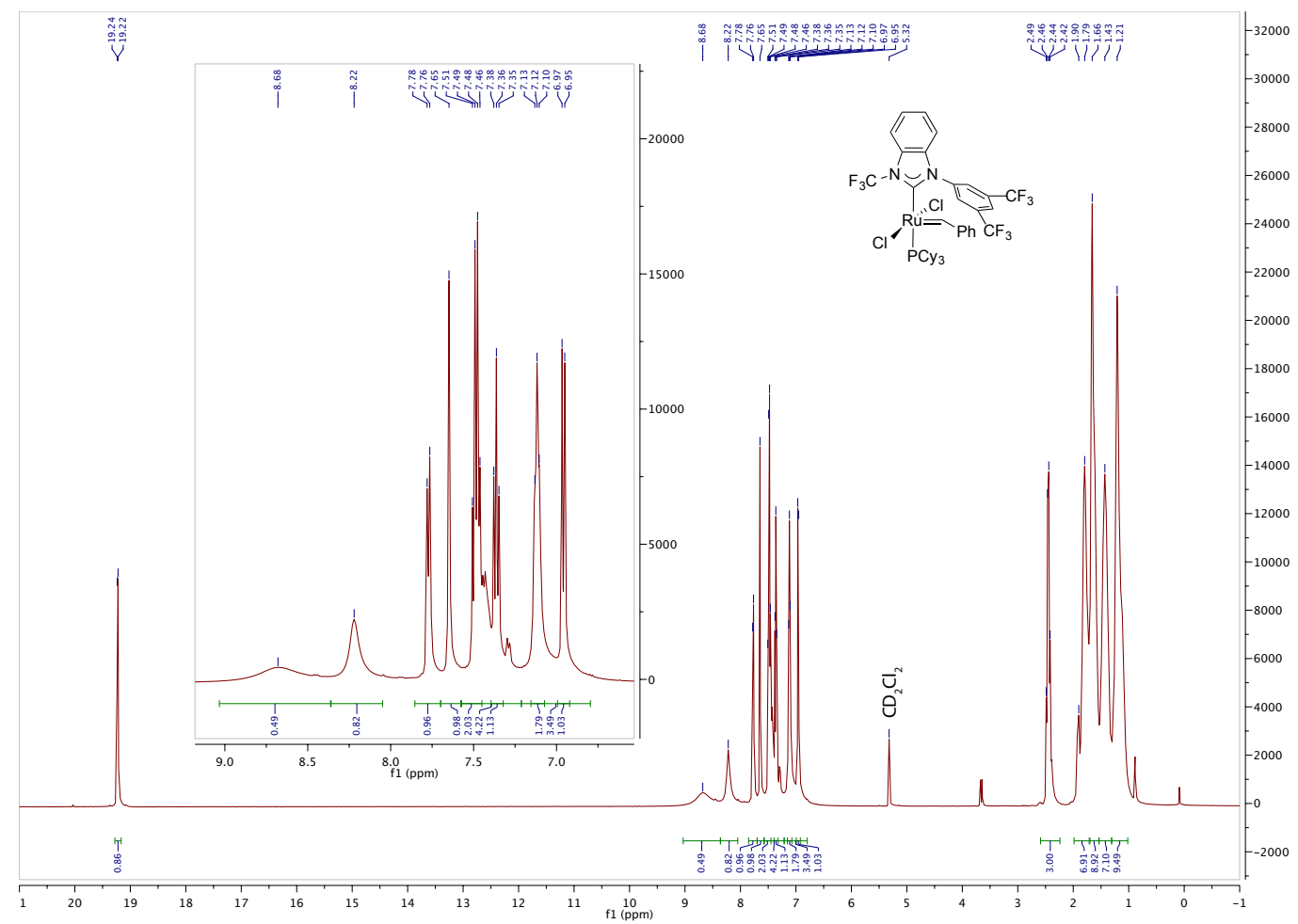

${ }^{19}$ F NMR (470.67 MHz, $\left.\mathrm{CD}_{2} \mathrm{Cl}_{2}\right)$ :

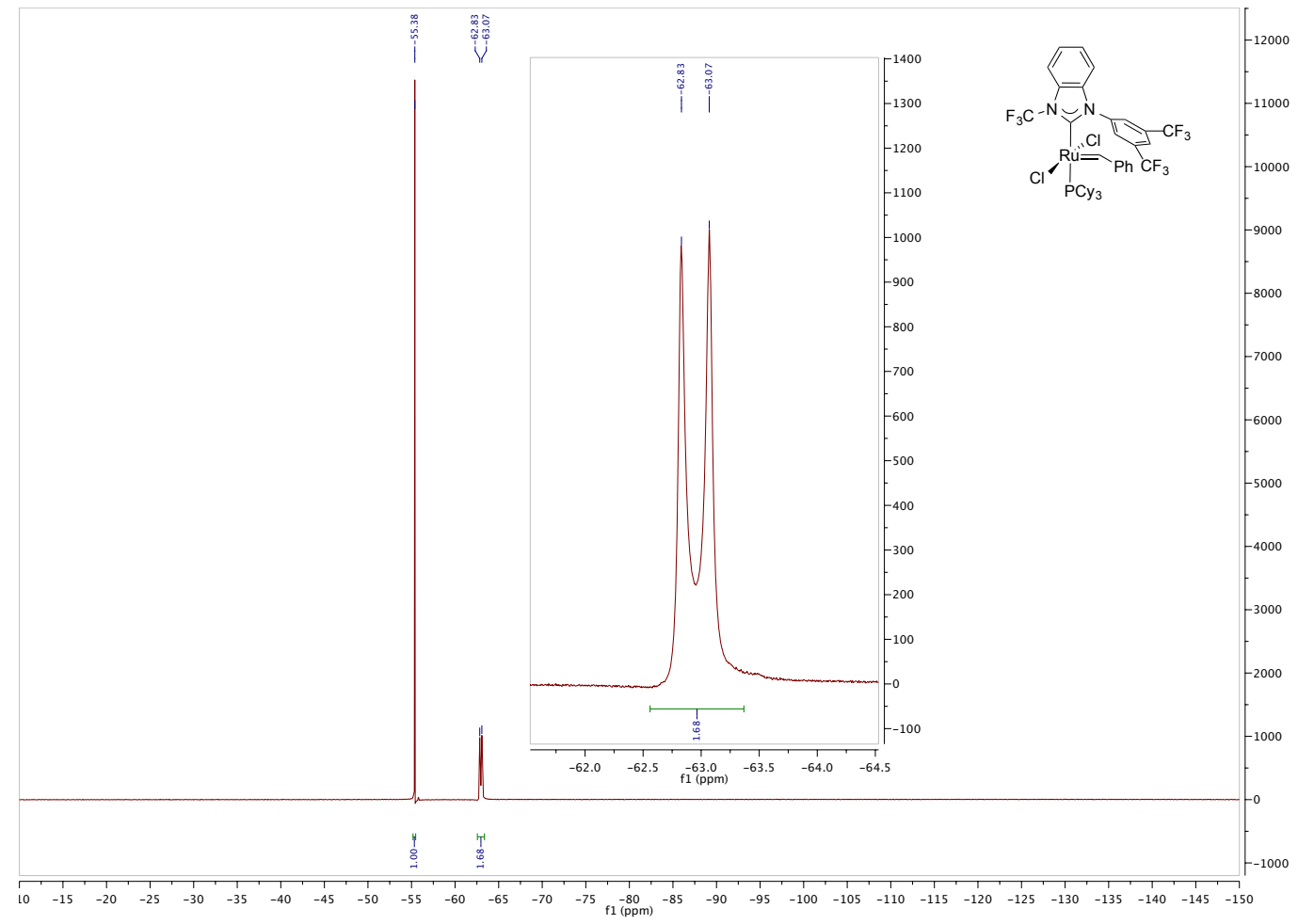


Supporting Information for Engl, Fedorov, Copéret, Togni

${ }^{19} \mathrm{~F} /{ }^{13} \mathrm{C}-\mathrm{HMBC}$ NMR (376.48/100.62 MHz, $\left.\mathrm{CD}_{2} \mathrm{Cl}_{2}\right)$ :

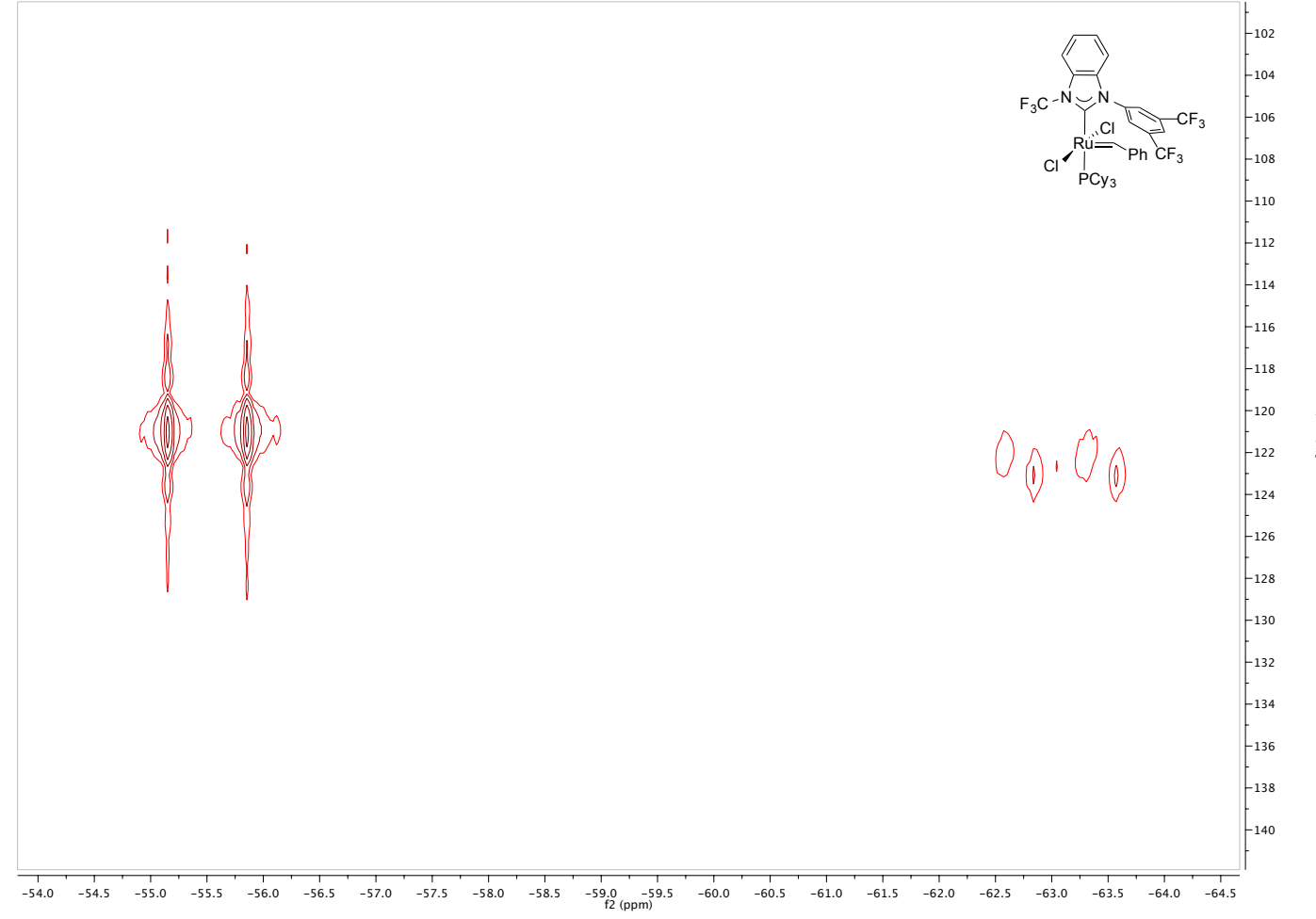

${ }^{31} \mathrm{P}$ NMR (202.50 MHz, $\left.\mathrm{CD}_{2} \mathrm{Cl}_{2}\right)$ :

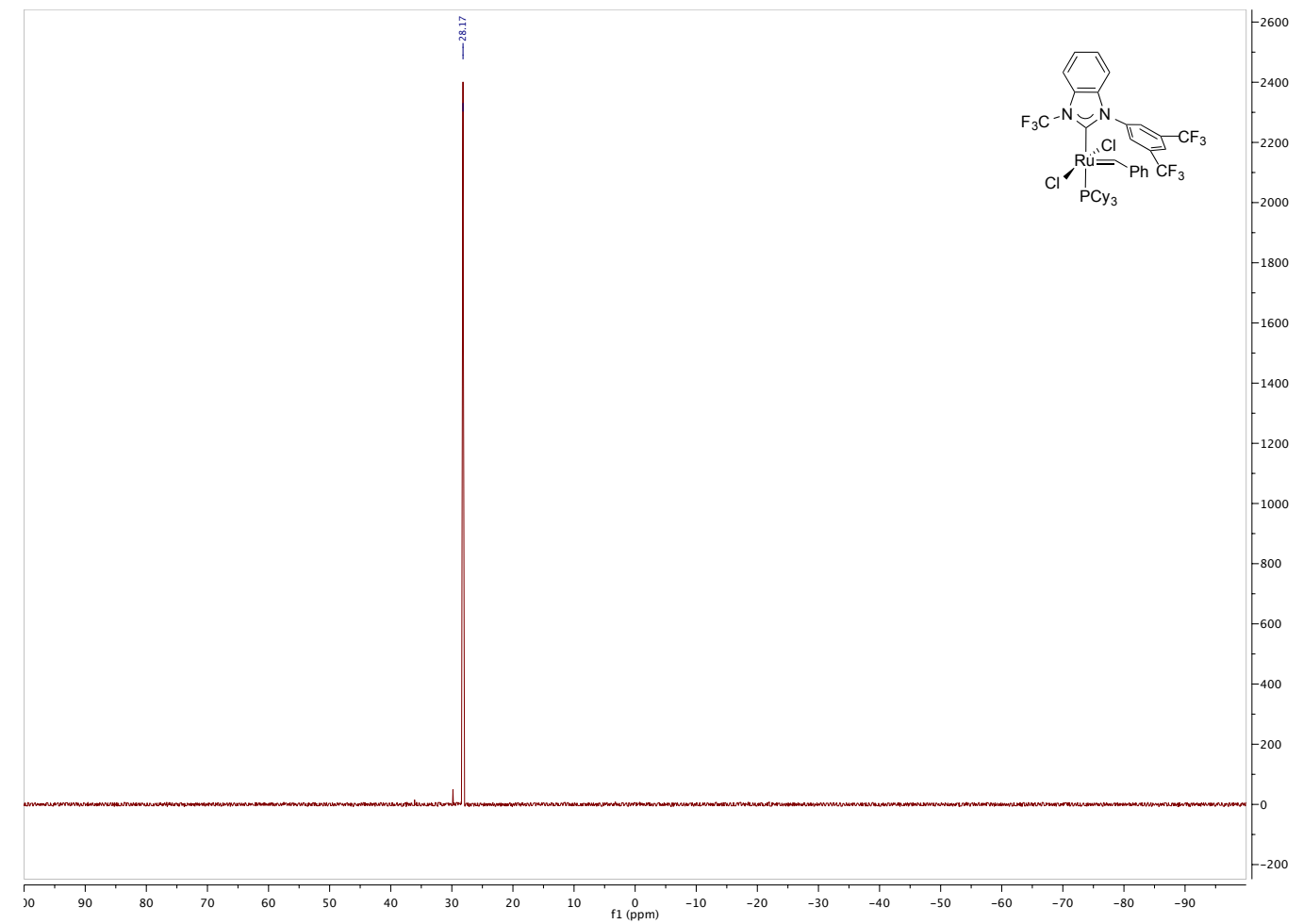


${ }^{13} \mathrm{C}\left\{{ }^{1} \mathrm{H}\right\}$ NMR $\left(125.81 \mathrm{MHz}, \mathrm{CD}_{2} \mathrm{Cl}_{2}\right)$ :

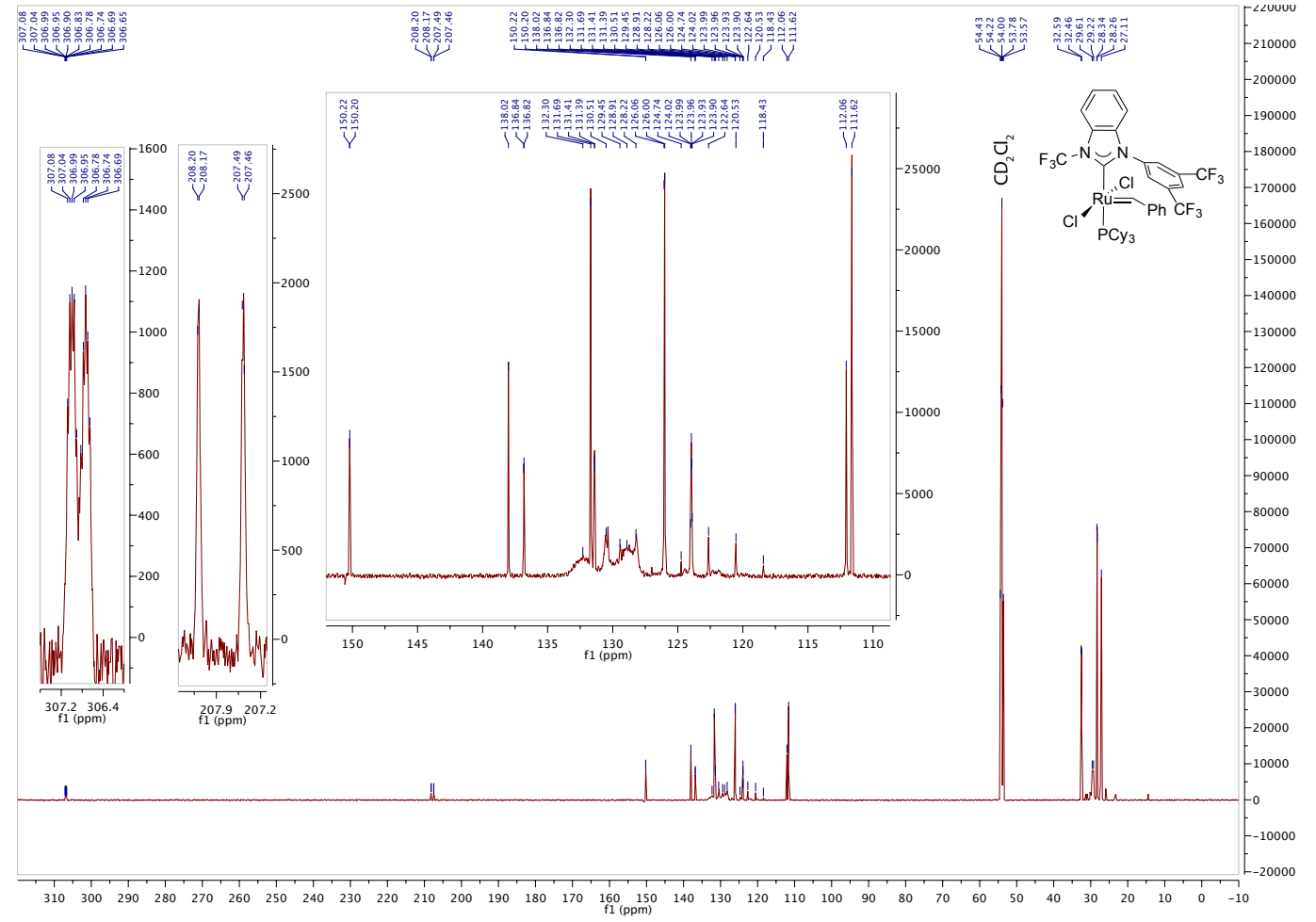

5.8 Tricyclohexylphosphine[3-butyl-1-(trifluoromethyl)- $1 H$-benzo[ $d \boldsymbol{d}]$ imidazol3-ylidene][benzylidene] ruthenium dichloride (6d)

${ }^{1} \mathrm{H}$ NMR (500.27 MHz, $\left.\mathrm{CD}_{2} \mathrm{Cl}_{2}\right)$ :

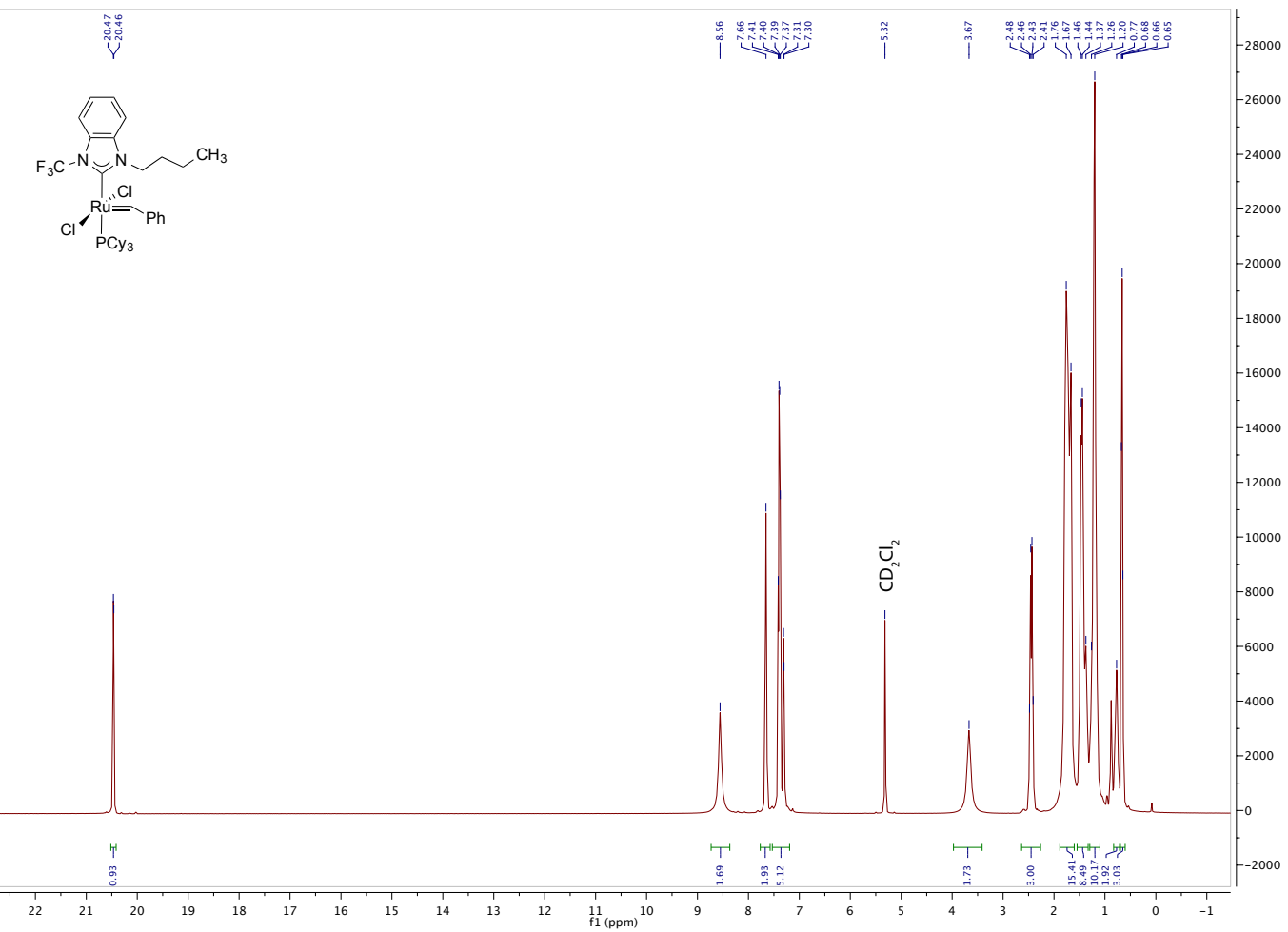


Supporting Information for Engl, Fedorov, Copéret, Togni

${ }^{19} \mathrm{~F}$ NMR (470.67 MHz, $\mathrm{CD}_{2} \mathrm{Cl}_{2}$ ):

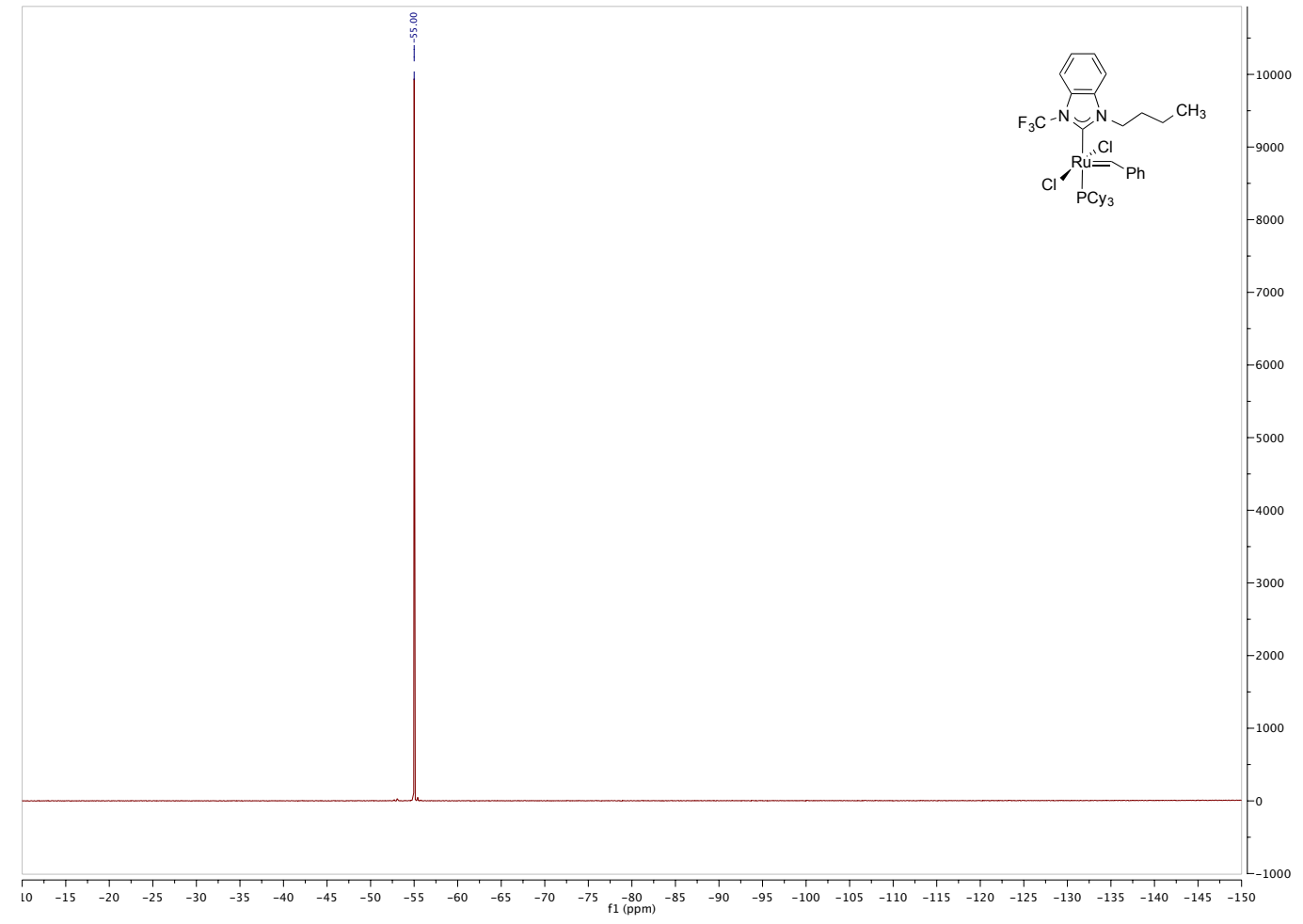

${ }^{31} \mathrm{P}$ NMR (202.50 MHz, $\left.\mathrm{CD}_{2} \mathrm{Cl}_{2}\right)$ :

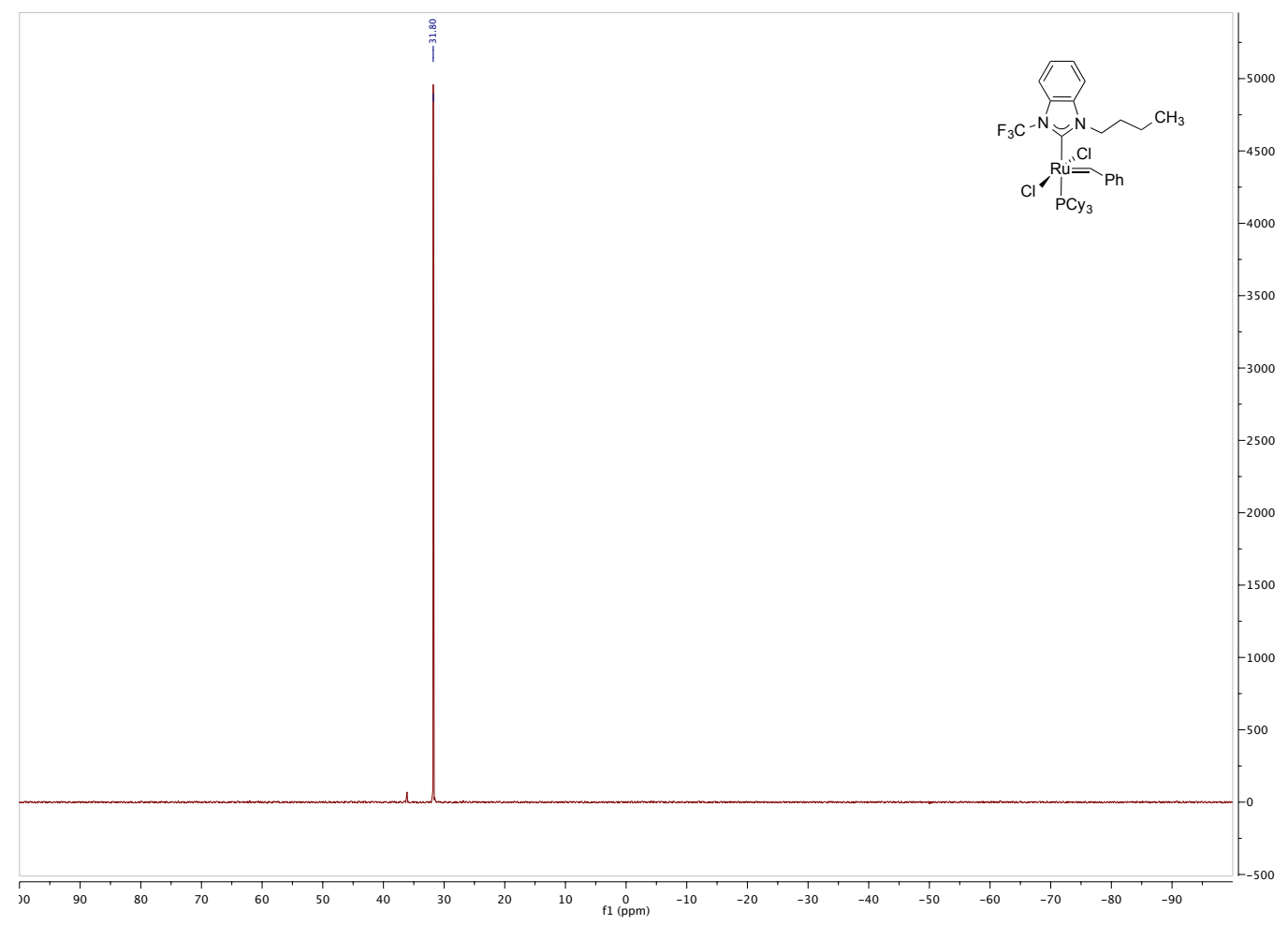


${ }^{13} \mathrm{C}\left\{{ }^{1} \mathrm{H}\right\}$ NMR $\left(125.81 \mathrm{MHz}, \mathrm{CD}_{2} \mathrm{Cl}_{2}\right)$ :

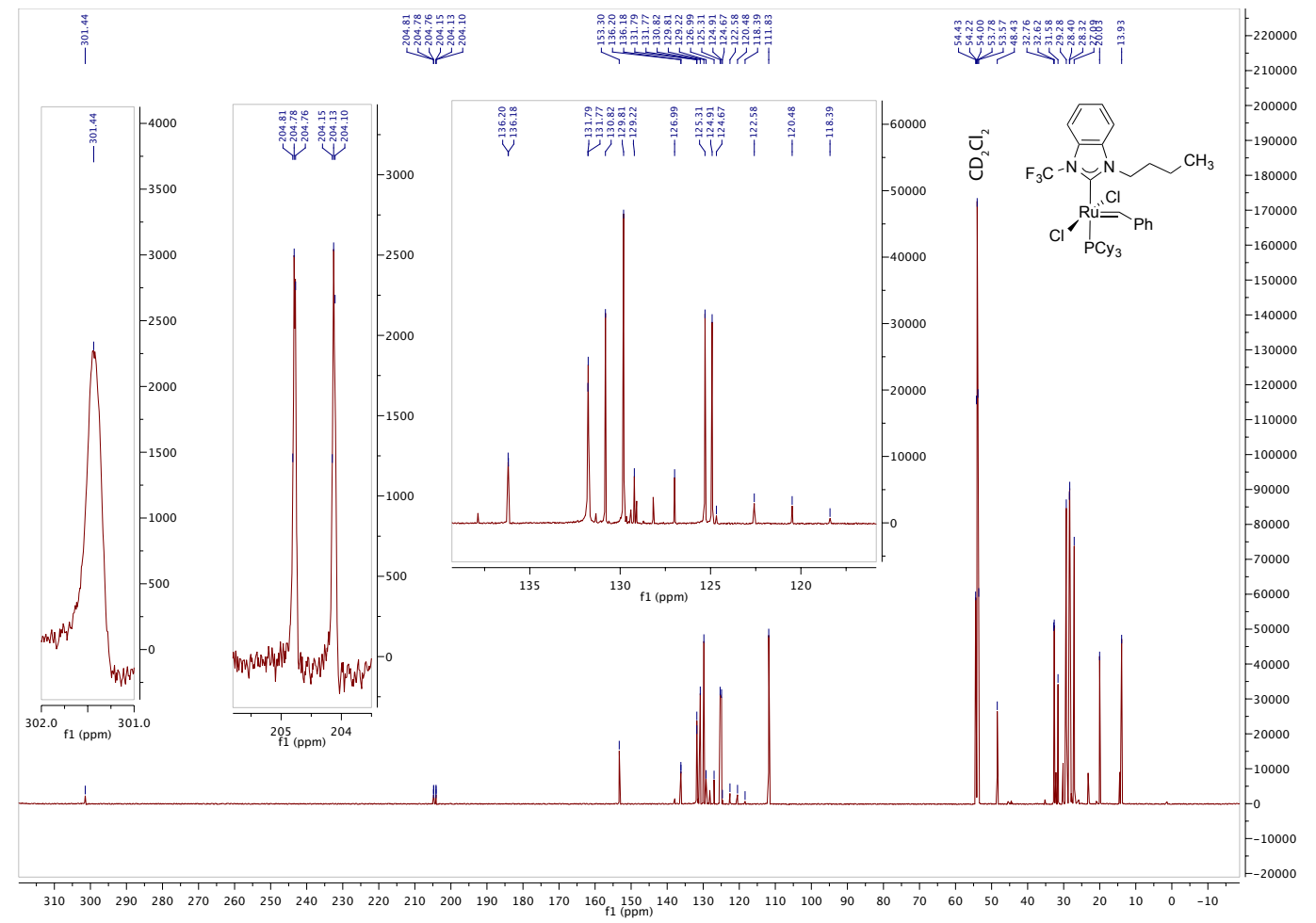

5.9 Tricyclohexylphosphine[1-isopropyl-3-(4-(trifluoromethyl)phenyl)-1Hbenzo $[d]$ imidazol-3-ylidene][benzylidene]ruthenium dichloride (7).

${ }^{1} \mathrm{H} \mathrm{NMR}\left(500.27 \mathrm{MHz}, \mathrm{CD}_{2} \mathrm{Cl}_{2}\right)$ :

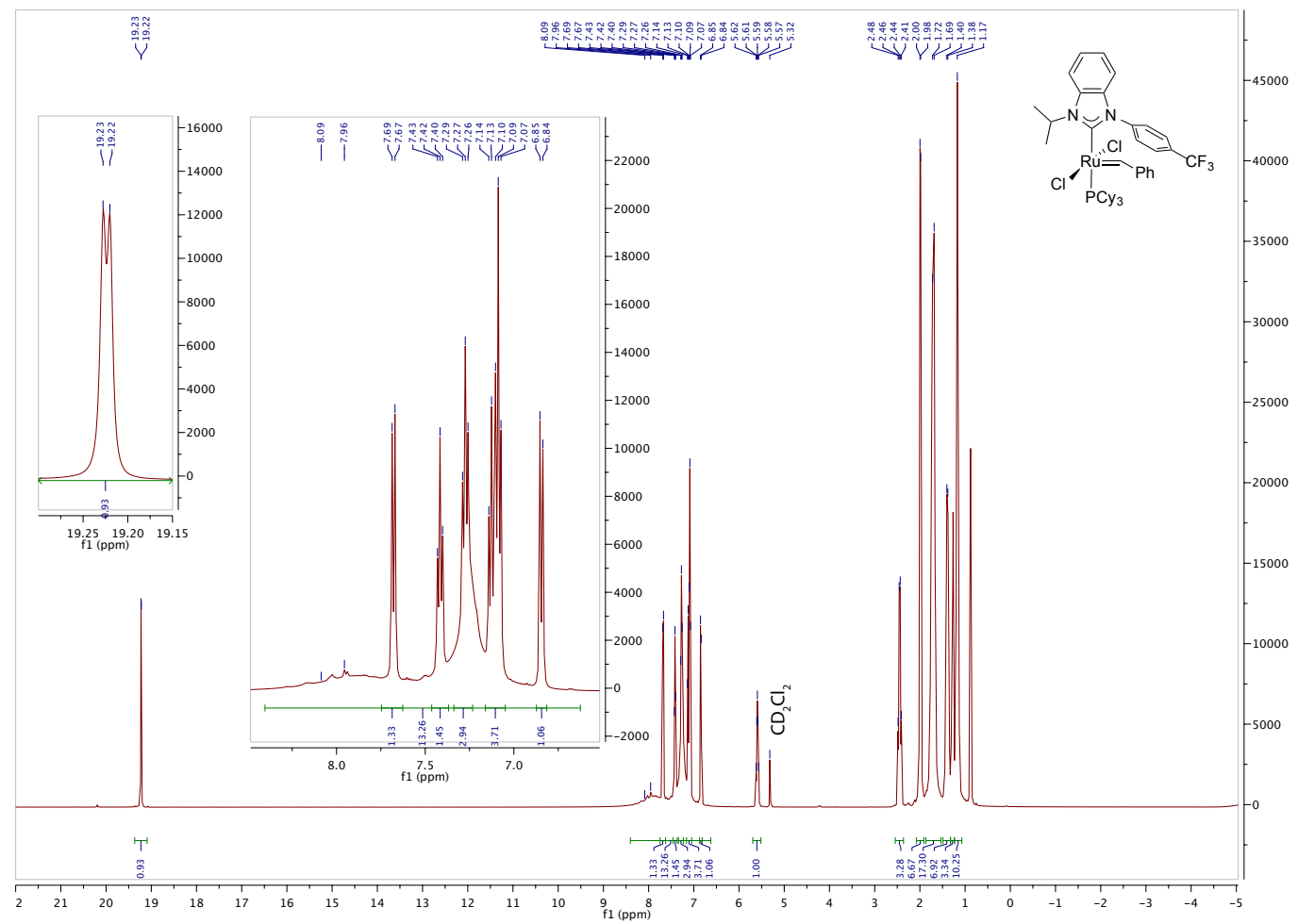


Supporting Information for Engl, Fedorov, Copéret, Togni

${ }^{19} \mathrm{~F}$ NMR $\left(470.67 \mathrm{MHz}, \mathrm{CD}_{2} \mathrm{Cl}_{2}\right)$ :

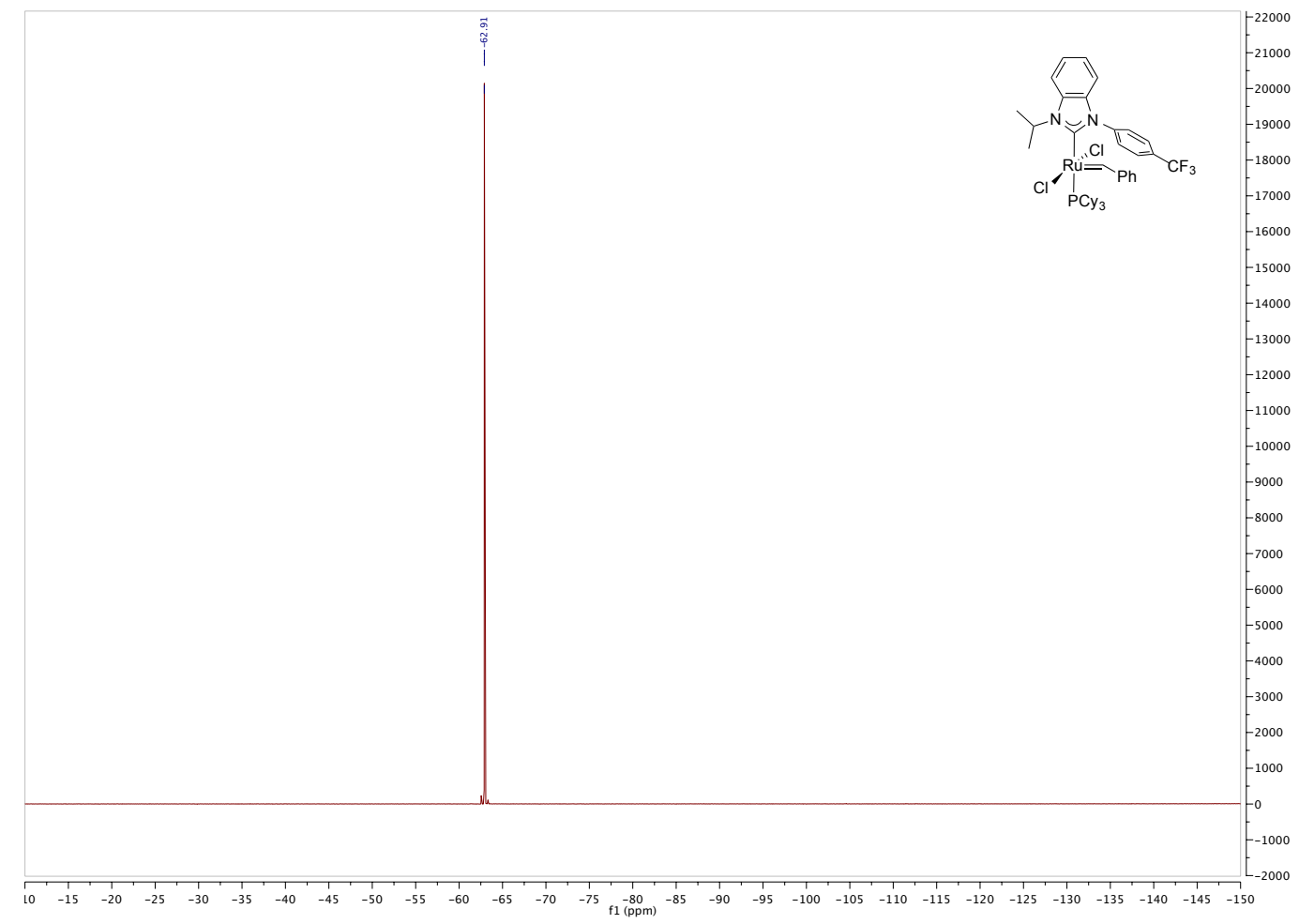

${ }^{31} \mathrm{P}$ NMR (202.50 MHz, $\left.\mathrm{CD}_{2} \mathrm{Cl}_{2}\right)$ :

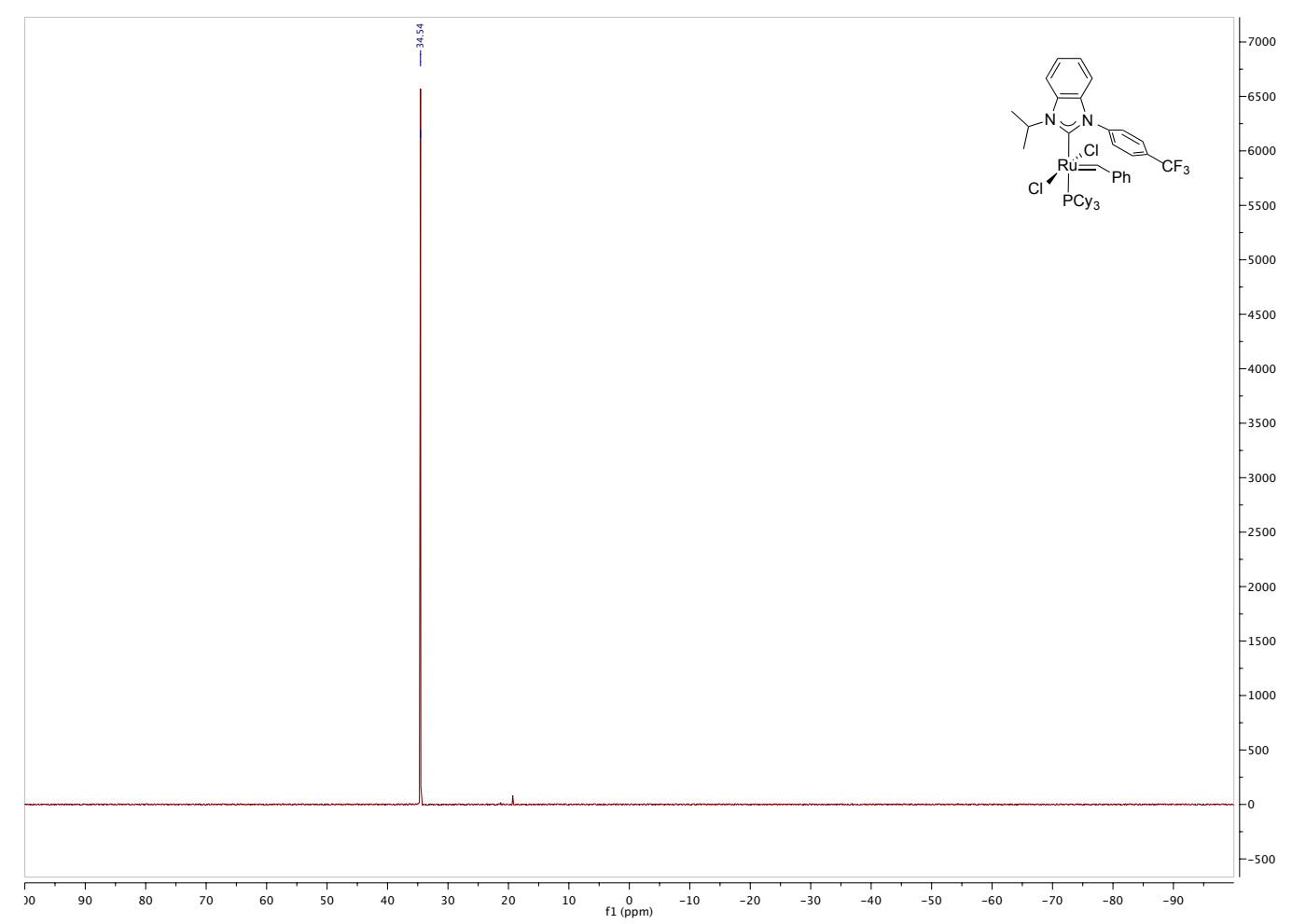


${ }^{13} \mathrm{C}\left\{{ }^{1} \mathrm{H}\right\}$ NMR (125.81 MHz, $\left.\mathrm{CD}_{2} \mathrm{Cl}_{2}\right)$ :

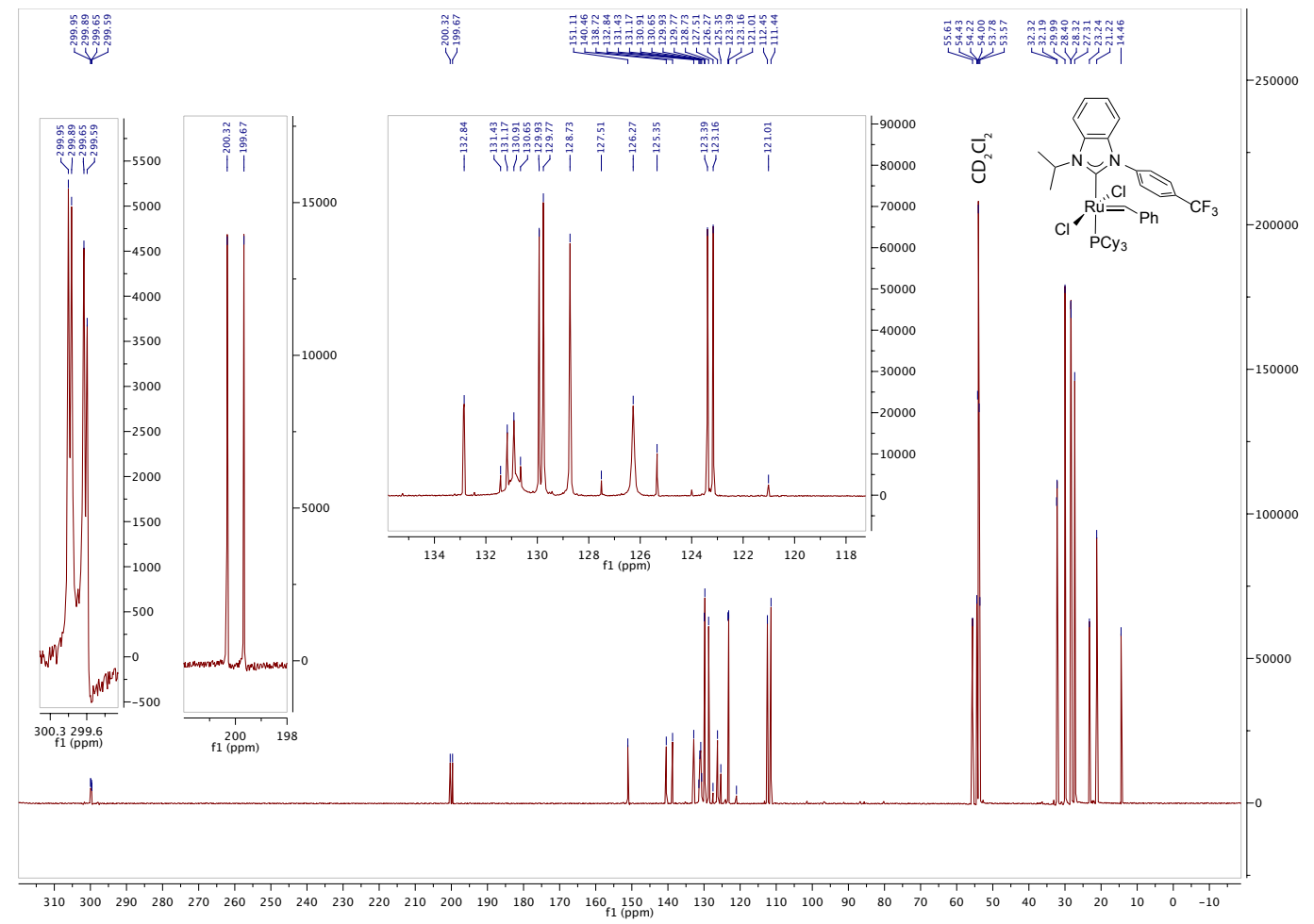

\section{References}

1. Engl, P.; Senn, R.; Otth, E.; Togni, A.; Organometallics 2015, 34, 1384 - 1395. 